\title{
Revised Mineralogic Summary of Yucca Mountain, Nevada
}

\author{
D. L. Bish \\ S. J. Chipera
}

\section{DISCLAIMER}

This report was prepared as an account of work sponsored by an agency of the United States Government. Neither the United States Government nor any agency thereof, nor any of their employees, makes any warranty, express or implied, or assumes any legal hability or responsibility for the accuracy, completeness, or usefulness of any information, apparatus. product, or process disclosed, or represents that its use would not infringe privately owned rights. Reference herein to any specific commercial product, process, or service by trade name. trademark. manufacturer, or otherwise does not necessarily constitute or imply its endorsement, recommendation, or favoring by the United States Government or any agency thereof The views and opinions of authors expressed herein do not necessarily state or reflect those of the United States Government or any agency thereof 
REVISED MINERALOGIC SUMMARY OF YUCCA MOUNTAIN, NEVADA

by

D. L. Bish and S. J. Chipera

\begin{abstract}
We have evaluated the three-dimensional mineral distribution at Yucca Mountain, Nevada, using quantitative $x$-ray powder diffraction analysis. All data were obtained on core, cuttings, or sidewall samples obtained from drill holes at and around Yucca Mountain. Previously published data are included with corrections, together with new data for several drill holes. The data in this report therefore supersede previously published quantitative $x$-ray diffraction data for these samples. The new data presented in this report used the internal standard method of quantitative analysis, which yields results of high precision for the phases commonly found in Yucca Mountain tutts, including opal-CT and glass. Mineralogical trends with depth previously noted are clearly shown by these new data. Glass occurrence is restricted almost without exception to above the present-day static water level (SWL), although glass has been identified below the SWL in partially zeolitized tufts. Silica phases undergo well-defined transitions with depth, with tridymite and cristolialte occurring onty above the SWL, opal-CT occurring with clinoptilolite-mordenite tutfs, and quartz most abundant below the SWL. Smectite occurs in small amounts in most samples but is enriched in two distinct zones. These zones are at the top of the vitric nomwelded base of the Tiva Canyon Member and at the top of the basal vitrophyre of the Topopah Spring Member. Our data support the presence of several zones of mordenite and clinoptilolite-heulandite as shown previously. New data on several deep clinoptilolite-heulandite samples coexisting with analcime show that they are heulandite. Phillipsite has not been found in any Yucca Mountain samples, but erionite and chabazite have each been found once in fractures. Laumontite has been positively identified in several samples from drill hole UE-25p\#1 and may also occur near the bottom of USW G-1.
\end{abstract}

\title{
I. INTRODUCTION
}

The Yucca Mountain Project, formerly the Nevada Nuclear Waste Storage Investigations (NNWSI) Project, of the U.S. Department of Energy has been conducting an on-going study of Yucca Mountain and surroundings to assess the suitability of the area to host the nation's first high-level radioactive waste repository. Research on this Project has pursued numerous avenues. One of the broad studies of fundamental importance in assessing the ability of the site to pertorm adequately as a geologic repositon 
is that of the mineralogy and petrology of the rocks at Yucca Mountain. Complete, three-dimensional information is required on the rock types and properties and on the distribution of all minerals at Yucca Mountain. These data are required to permit optimal siting of a repository and accurate prediction of the possible interactions between heat and waste trom the potential repository and the rocks and minerals. In addition, because the primary potential mechanism of radionuclide migration to the accessible environment is via groundwater transport, accurate data are required concerning the nature of the rocks and minerals along potential flow paths away from the repository. Los Alamos National Laboratory has given considerable effort to characterize and understand the mineralogy and petrology of the rocks at and surrounding Yucca Mountain (Heiken and Bevier 1979; Sykes et al. 1979; Bish 1981; Bish et al. 1981; Caporuscio et al. 1982; Vaniman et al. 1984; Bish and Vaniman 1985). This report summarizes our current knowledge of the three-dimensional mineralogy at Yucca Mountain. The report contains some data previously published (Bish and Vaniman 1985) and corrects printing errors that were contained therein. In addition, this report contains new data for drill holes USW G-1, J-13, UE-25a\#1 (Bish and Chipera 1986) and UE-25p\#1 (Chipera and Bish 1988). The data presented here therefore update previously published $x$-ray data, but the mineralogic cross sections presented in Appendix 8 of Bish and Vaniman (1985) have not been reconstructed and remain valid because of their schematic nature.

\section{ANALYTICAL METHODS}

The x-ray data presented in Appendix A were obtained primarily from core samptes, with some data obtained from either drill-cutting or sidewall samples. The locations of the drill holes studied are shown in Fig. 1. Depths are known for both core and sidewall samples, but depths for drill cuttings are approximate within a range of about $10 \mathrm{ft}(3.28 \mathrm{~m})$. Older $\mathrm{x}$-ray data were obtained on samples ground under acetone to pass through a 325-mesh (45- $\mu \mathrm{m}$ ) sieve, but data for drill holes J-13, USW WT-1, USW WT-2, USW G1, UE-25a"1, and UE-25p\#1 were obtained on samples ground under acetone to less than $5 \mu \mathrm{m}$ in an automatic Brinkmann Retsch mill with an agate mortai and pestle. The particle size disiributions of these newer samples were veritied using a Horiba CAPA-500 centritugal particle size distribution analyzer calibrated with glass microsphere standards. This fine crystallite size is necessary to ensure adequate particle statistics and to minimize primary extinction (Kkug and Alexander 1974, pp. 365-367). A portion of most of the newer samples was mixed with a $1.0-\mu \mathrm{m}$ corundum $\left(\mathrm{Al}_{2} \mathrm{O}_{3}\right)$ internal standard in the ratio $80 \%$ sample to $20 \%$ internal standard. These samples analyzed with an internal standard are designated with an "I" in Appendix $A$ in a column labeled "method." Those samples with no method designation in Appendix A were analyzed using the external standard method (see below). The powders resulhing from the above preparation treatment were mounted in a $22-x 44-\mathrm{mm}$ cavity in a glass slide; this sample area is sifficient for the sample to contain the $x$-ray beam as low as $8.0^{\circ} 2 \theta$. Powder samples from USW G-4 were mounted in a sample spinner on the diffractometer; all other samples were examined stationary. 


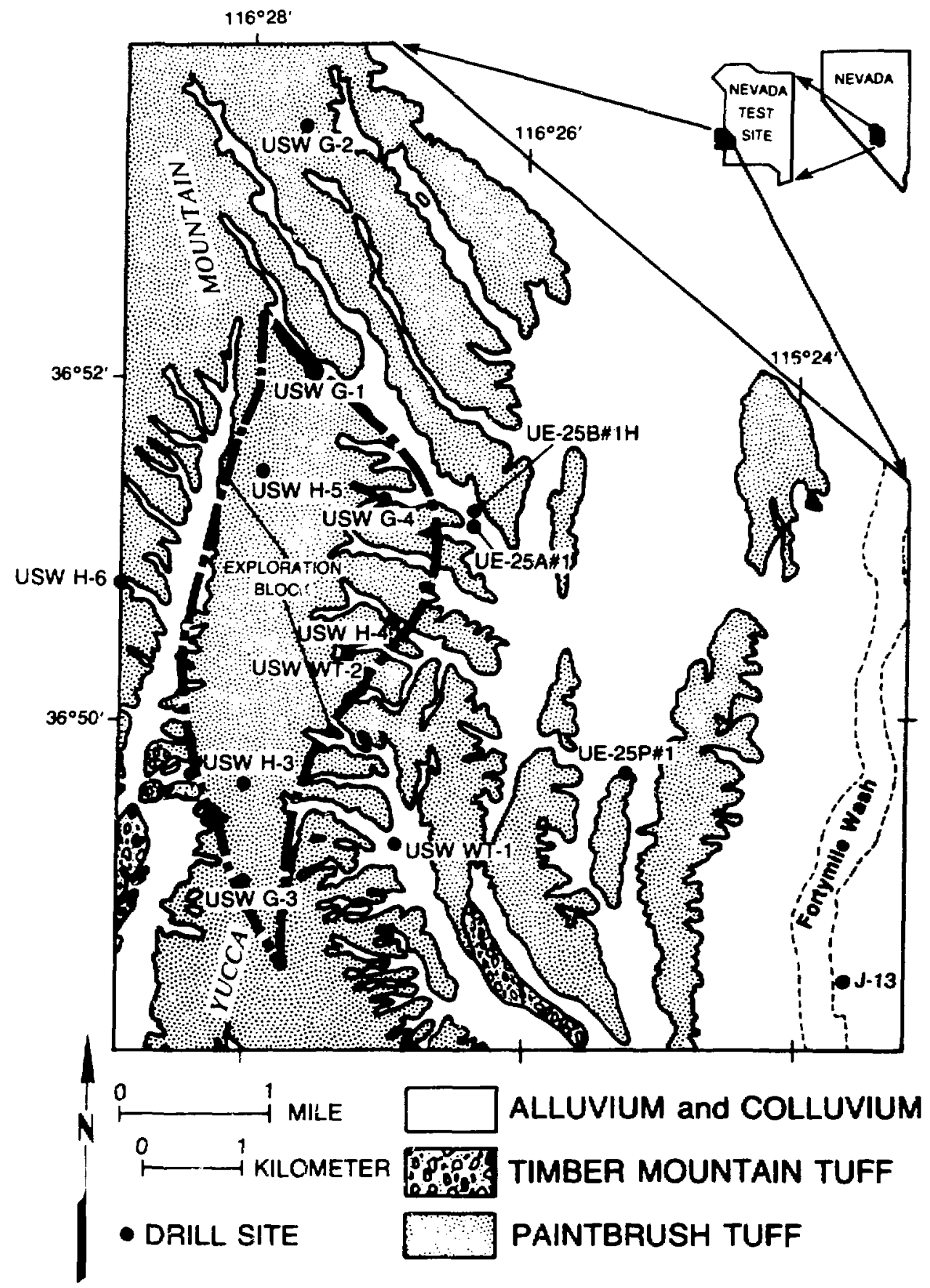

Fig. 1. Location map of Yucca Mountain, Nevada, showing the outline of the exploration block for the NNWSI Project and the locations of drill holes mentioned in the text. Drill hole $\mathrm{J}-12$ is located outside of this figure to the southeast and can be found on the location map published in Bish et al. (1984). 
. All x-ray powder diffraction data were oblained on an automated Siemens D-500 diffractometer using Cu-Ka radiation, a graphite diffracted-beam monochromator, and pulse-height analysis. Data from USW G-2 and UE-25b\#1H were collected manually, scanning from $2.0^{\circ}$ to $36.0^{\circ} 2 \theta$ at a rate of $1 \% / \mathrm{min}$. All other data were collected automatically in the step-scan mode, with a step size of $0.02^{\circ} 2 \theta$ and coum times of at least $2.0 \mathrm{~s}$ per step. Data for samples with no internal standard were collected from $2.0^{\circ}$ to $36.0^{\circ} 2 \theta$, and data for samples with an added conundum internal standard were collected from $2.0^{\circ}$ to $50.0^{\circ} 2 \theta$. Several samples were examined using count times as long as $55 \mathrm{~s}$ per step to improve detection limits for trace phases.

Mineral identification was accomplishec by comparison of observed diftraction patterns with standard patte ne reasured in this laboratory, by comparison with published standards from the Joint Committee on Pow ter ? iffraction Standards (JCPDS), and by comparison with patterns calculated using the program POWD10 (Smith et al. 1982). Clay mineral standards were obtained from the Clay Minerats Society Source Clay Repository, and zeolite standards were obtained from the Minerals Research Corporation, Clarkson, New York. Differentiation between kaolinite and chlorite was accomplished by heating, but admixed smectite and low amounts of chlorite/kaolinite often gave ambiguous results. Hematite identification was based on major peaks at $33.2^{\circ}$ and $35.7^{\circ} 2 \theta$. However, overlap with zeolite peaks mado the unambiguous determination of the presence of hematite impossible in zeolitized samples.

\section{A. Quantitative Analyses}

Qualitative identification of the crystalline phases present in tutfs can be accomplished quickly and accurately using $x$-ray powder diffraction. The factors making optical identification of minerals ambiguous, such as the very fine-grained and intergrown texture of the tuffs, do not affect $x$-ray powder diffraction analyses. However, quantitative multicomponent analysis of rock samples is not straightforwand and ks complicated by numerous tactors (Bish and Chipera 1988). These factors inchude variations in degree of preferred orientation of crystallites, variations in crystalite size, variations in degree of crystallinity (cryetal perfection), and variations in composition (solid-solution effects). In addition, the diffraction patterns from these complex mixtures of minerals often show peaks from at least six different phases. Thus the method of obtaining intensities from these complex patterns with numerous overlapping peaks can signilicanty affect quantitative results. Finally, because all of our quantitative analyses require the use of standards, the choice of standards plays a crucial role. It is important that standards be as similar to the minerals in the rocks as possible.

All of our older analyses and several recent analyses of samples for which amounts were insufficient for the internal standard method employed the external standard or adiabatic method of Chung $\{1974 a, b\}$. However, whenever sutficient sample was available, our recent analyses employed the internal standard

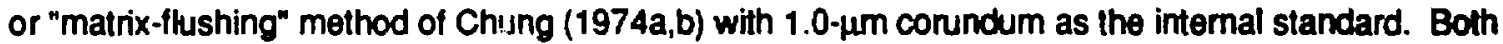
methods require that reference intensity ratios (RIRs) be determined belore quantitative analysis. We 
have therefore experimentally determined $R ! R s$, the ratio of the integrated intensity of a given reflection of the phase of interest to the integrated intensity of the 113 reflection of conndum in a $1: 1$ mixture by weight, for most phases found in Yucca Mountain tutts. The 1:1 ratio of standard to sample was chosen for convenience. In practice any ratio can be used for standardization because the relative amounts of standard and sample are incorporated into the reduction of standard runs. RIR values used in our analyses are given in Table I, together with sources of materials. In the absence of sufficient pure material, RIRs for some phases were calculated using the program POWD10 (Smith et al. 1982).

All of the experimentally measured RIR values for standards in Table I represent the average of six repetitions of the standard-plus-corundum mixture. The values in Table I are strictly applicable only to data obtained on our Siemens diffractometer in its current configuration. Modifications of the instrument or of the sample preparation and mounting method will ideally require remeasurement of RIRs for ali phases.

The RIR value for smectite was cbtained from material separated from USW G-1 1415 (431.3 m). and this RIR was used for most analyses. However, samples below $3400 \mathrm{ft}(1036 \mathrm{~m})$ in USW G-1 showed evidence for some chloritic interlayers, and the RIR for these samples was obtained from the smectite from USW G-1 3490 (1063.8 m). Smectite analyses for UE-25p\#1 used RIRs measured on smectites separated from samples at different depths within the UE-25p\#1 drill hole. Older analyses employed an RIR for clinoptilolite measured for a clinoptilolite from the 1660-t! $(506-\mathrm{m})$ depth in drill hole UE-4P at the Nevada Test Site. Recent RIRs for clinoptilolite were measured for five different samples to assess the effects of composition and preferred orientation on RIR (Table I). There is an approximately linear relationship between the RIR for the $9.8^{\circ}(020)$ reflection and the intensity ratio of the $9.8^{\circ}$ reflection to the sum of the reflections between $22.4^{\circ}$ and $22.7^{\circ} 2 \theta$ (Fig. 2). Thus our recent analyses (USW G-1. UE$25 a \# 1, J-13$, and UE-25p\#1) used the ratio of the $9.8^{\circ}$ peak to the sum of the $22.4^{\circ}-22.7^{\circ} 2 \theta$ peaks to determine the RIR of the $9.8^{\circ} 20020$ reflection and used a fixed RIR for the $22.4^{\circ}-22.7^{\circ} 2 \theta$ cluster. When the intensity between $22.4^{\circ}$ and $22.7^{\circ} 2 \theta$ was not measurable, we used a value of 0.97 for the $9.8^{\circ} 2 \theta 020$ RIR. We compensate for the effects of preferred orientation and chemistry on the mordenite diffraction pattern by using a procedure similar to that used for clinoptilolite. We experimentally determine the ratio of the mordenite $9.8^{\circ}$ and $19.6^{\circ} 2 \theta$ peaks and then use an appropriate RIR. However, mordenite usually coexists with clinoptilolite, making use of the $9.8^{\circ} 2 \theta$ mordenite peak impossible because of interference with the $9.8^{\circ} 2 \theta$ clinoptilolite 020 reflection. In these cases, we use an RIR for the $19.6^{\circ} 2 \theta$ peak or 0.20 . The strong $25.6^{\circ} 2 \theta$ mordenite reflection cannot be easily used because of interference with both feldspar and corundum internal standard reflections. Analcime has little compositional variability, so we use an RIA measured on a single natural sample. 
TABLE I

REFERENCE INTENSITY RATIOS (RIRO) FOR MINERALS ANALYSED

\begin{tabular}{|c|c|c|c|}
\hline MNERAL & SOURCE & $\begin{array}{l}\text { REFLECTION } \\
h h l(2 \theta \text { mang) }\end{array}$ & RIP \\
\hline Analcime & W'kieup, AZ & $\begin{array}{ll}211 & (15.8) \\
400 & (26.0)\end{array}$ & $\begin{array}{l}1.27 \\
1.80\end{array}$ \\
\hline Calclte & Lab Reagent & $\begin{array}{ll}104 & (29.4) \\
116 & (48.5)\end{array}$ & $\begin{array}{l}2.96 \\
0.58\end{array}$ \\
\hline Chlorite & $\begin{array}{l}\text { Flagstaff Hill, CA, } \\
\text { Ripidolite }\end{array}$ & $\begin{array}{cc}001 & (6.1) \\
002 & (12.4) \\
004 & (25.1)\end{array}$ & $\begin{array}{l}1.53 \\
3.14 \\
1.74\end{array}$ \\
\hline Clinoptilolite & $\begin{array}{l}25525 \text { Castle Creek, } 10 \\
27032 \text { Castle Creek, } 10 \\
27073 \text { Sheaville, OR } \\
27083 \text { Buckhorn, NM } \\
\text { UE4P-1660 Yucca Flat, NV }\end{array}$ & $\begin{array}{c}020(9.8) \\
(22.4-22.7) \\
(30.0)\end{array}$ & $\begin{array}{l}0.97 \\
1.06 \\
0.49\end{array}$ \\
\hline Cristobalite & Symthetic Cristobalite & $\begin{array}{ll}101 & (21.9) \\
200 & (36.1)\end{array}$ & $\begin{array}{l}4.91 \\
0.84\end{array}$ \\
\hline Dolomite & $\begin{array}{l}\text { UE-25p\#1-4313/4318, } \\
\text { Yucca Mt, NV }\end{array}$ & $\begin{array}{ll}104 & (30.9) \\
113 & (41.1)\end{array}$ & $\begin{array}{l}2.69 \\
0.48\end{array}$ \\
\hline $\begin{array}{l}\text { Feldspar } \\
\text { Albite } \\
\text { Albite } \\
\text { Sanidine } \\
\text { Sanidine }\end{array}$ & $\begin{array}{l}\text { Amelia, VA } \\
\text { Amelia, VA } \\
\text { Eifel, Germany } \\
\text { Eifel, Germany }\end{array}$ & $\begin{array}{c}(13.4-14.0) \\
111(23.6) \\
(13.4-14.0) \\
130(23.6)\end{array}$ & $\begin{array}{l}0.24 \\
0.27 \\
0.13 \\
0.55\end{array}$ \\
\hline Fluorite & Lab Reagent & $\begin{array}{ll}111 & (28.3) \\
220 & (47.0)\end{array}$ & $\begin{array}{l}3.24 \\
3.70\end{array}$ \\
\hline Gypsum & Wyoming Selenite & $020 \quad(11.5) 4$ & 4.25 \\
\hline Hematite & Cleater Moor, England & $\begin{array}{ll}104 & (33.2) \\
110 & (35.7)\end{array}$ & $\begin{array}{l}2.38 \\
1.20\end{array}$ \\
\hline Hornblende & Yucca Mt, NV & $110(10.4)$ & 2.34 \\
\hline Hitite & Silver Hill, MT & (8.8) & 0.57 \\
\hline IImenite & Koidu, Sierra Leone & $\begin{array}{ll}104 & (32.8) \\
024 & (49.0)\end{array}$ & $\begin{array}{l}1.91 \\
0.62\end{array}$ \\
\hline
\end{tabular}




\begin{abstract}
Kaolinite
\end{abstract}
Laumontite

Magnetite

Mica

Mordenite

Opal-CT

Quartz

Smectite

Tridymite
Average of Wards' Kaolin,

Washington County, GA,

and Mesa Alta, NM

Collected by D. Bish in

Oregon, 1984

Ontario, Canada

Avg of calculated and

Bancroft, Ontario

Union Pass, AZ

Lovelock, NV

USW G1-2314, Yucca Mt, NV

28501 Kliwikerthal, Czech.

92509 Owyhee County, ID

Hoi Springs, AR

Yucca Mi, NV
$100 \quad(20.8)$

0.86

101 (26.6)

4.19

001

001

1.37

$110 \quad(9.3)$

1.15

0.47

0.59

0.78

2.54

3.8

$200 \quad$ (9.8)

0.44

0.20

0.36

1.80

(21.0-22.1)

3.85

1.73

(20.5)

(21.6)
1.18

1.10 


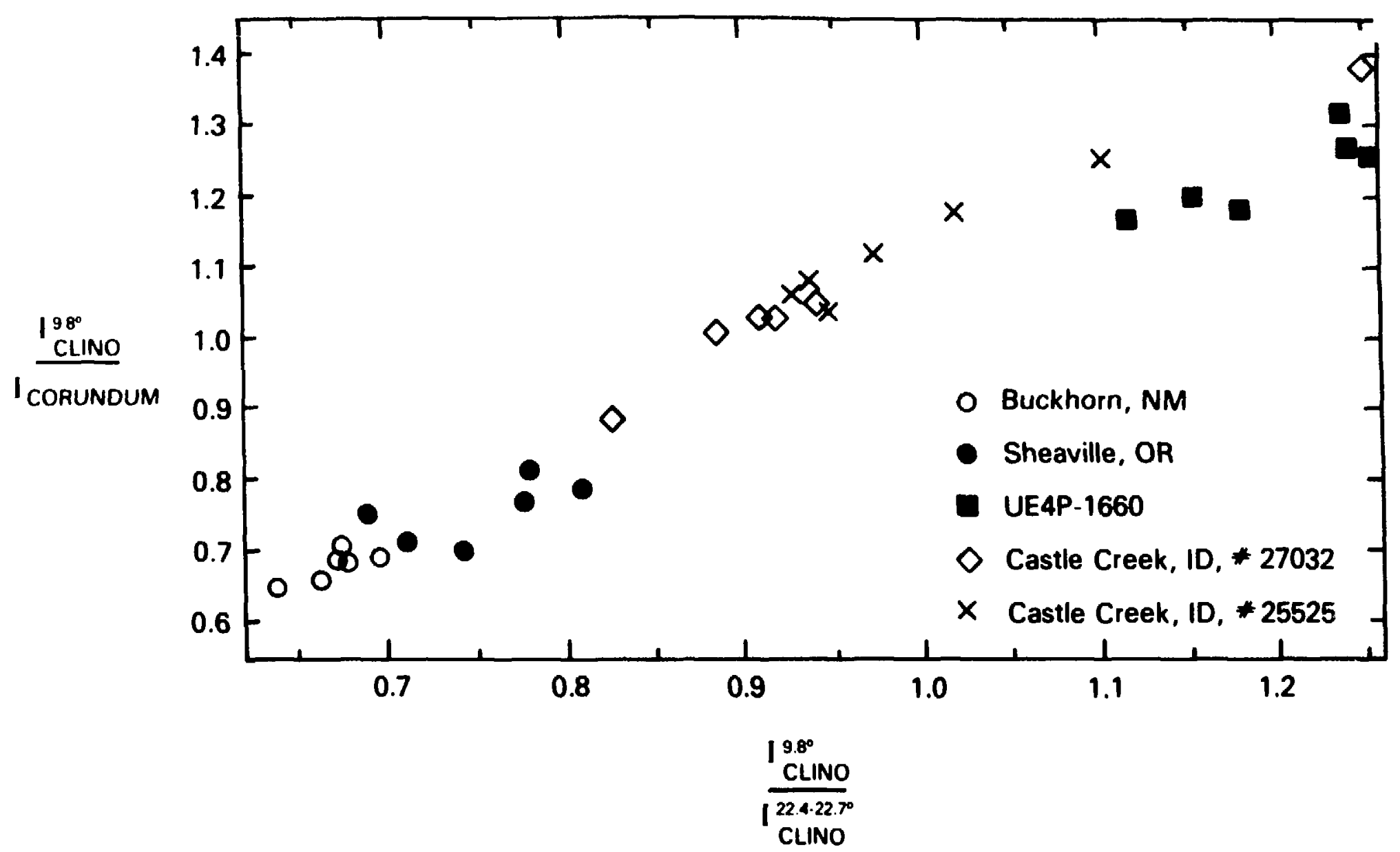

Fig. 2. Relationship between the RIR for the $9.8^{\circ}$ clinoptilolite peak and the intensity ratio of the $9.8^{\circ}$ reflection to the sum of the reflections between $22.4^{\circ}$ and $22.7^{\circ} 2 \theta$ (Cuk $\alpha$ ). 
Older analyses used a calculated RIR for tridymite, but recent analyses used an RIR measured on a tridymite-rich sample from Yucca Flat, NGvada Test Site, Nevada. An RIR for tridymite was chosen that yielded a matrix-flushing-analysis total of $100 \%$ for this sample, including the other crystalline phases present in the sample. The RIR for tridymite is thus of lower precision than RIRs for other samples that can be obtained in pure form. Older analyses included opal-CT with cristobalite, but recent analyses separated the two, using an RIR for opal-CT measured on two natural samples between $21.0^{\circ}$ and 22.1 $1^{\circ} \theta$. RIRs for alkali feldspar were obtained from natural albite and sanidine for peaks in the range $13.4^{\circ}$ to $14.0^{\circ} 2 \theta$ and at about $23.6^{\circ} 2 \theta$. These peaks are relatively free from interference from other phases, and if both peaks are used, the relative amounts of $\mathrm{K}$-feldspar and albite can be determined.

All of our analyses, apart from data collected manually, used integrated intensities rather than peak heights. Analysis of manually collected data (USW G-2, UE-25b\#1a) employed peak heights. The use of integrated intensities compensates for several sample-related problems, including variations in crystallinity and crystallite size. Most integrated intensities were obtained using the Siemens first-derivative peaksearch routine (IDENT) that yields precise intensities for resolved peaks. However, this routine divides the intensity of overlapping peaks at the midpoint between the two, which is only an approximation for most peaks. Closely overlapping or very broad peaks were measured by planimetry, and completely overlapping peaks were not decomposed. Overlapping tridymite and cristobalite peaks in samples from USW WT-1 and USW WT-2 were decomposed using a Siemens profile-refinement routine with a Gaussian profile function. However, more recent analyses employed manual planimetry, which we found to be superior to the profile-refinement results. Future analyses will probably employ the more-sophisticated profile-refinement program, FIT, in the latest Siemens software package, DIFFRAC 500.

As noted above, we solved the internal standard equation of Chung (1974a) to analyze for amounts of individual phases when an internal standard was used:

$$
x_{i}=\frac{x_{c}}{k_{i, n}} \frac{I_{i, n}}{I_{c}}
$$

where $X_{i}$ is the weight fraction of component $i, K_{i, n}$ is the RIR for line $n$ of component $i, X_{c}$ is the weight fraction of corundum in the sample (commonly $20 \%), l_{i, n}$ is the integrated intensity of line $n$ of component $i$, and $I_{C}$ is the integrated intensity of the corundum 113 reflection. It is important to note that this method does not require that the sum of the phases be equal to $100 \%$. Therefore, amounts of glass or other amorphous material such as opal-A can be determined by assuming that the difference between the total crystalline phases and $100 \%$ is due to amorphous components. There was petrographic evidence for glass in all samples in which we report glass. 
For older analyses and the recent analyses in which amounts of sample were insufficient for an intemal standard analysis, we solved the external standard equation derived by Chung (1974b):

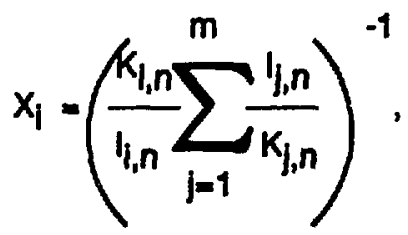

where $X_{i}$ is the unknown weight fraction of phase $i$ in the rock, $K_{i, n}$ is the RIR for line $n$ of phase $i, l_{i, n}$ and $j_{j, n}$ are the integrated intensity of line $n$ of phase $i$ and $j$, respectively, and $m$ is the number of phases in the mixture. This equation differs from Eq. (1) in that it is derived using the constraint that the sum of all phases equals $100 \%$. Both Eqs. (1) and (2) "flush" out the mass absorption coefficients by ratioing the RIRs for each phase. We have coded both the internal and the external standard methods, with all of the appropriate RIRs and correction routines in our computer program QUANT. The correction routines in QUANT include (1) correction of mordenite for overfap with smectite, feldspar, and corundum; (2) correction of cristobalite for overlap with abite and tridymite; (3) correction of corundum for overlap with calcite; (4) correction of clinoptiolite for overlap with mordenite; and (5) correction of clinoptilolite and opalCT for mutual overlap. In acdition, we have incorporated a routine correcting for compositional effects on the clinoptiblite RIR. In practice, these correctlons improve our accuracy and yield more realistic errors.

\section{B. Precision of Analyses}

Numerous factors affect the precision of our quantitative analyses. Some can be explicitly accommodated and others must be approximated. Factors attrlbutable to the sample or to the sample preparation process include solid solution, other variations in composition, and preferred orientation. Errors resulting from these factors are primarily attributable to differences between samples and standards. Other potentially important errors are encountered in data analysis, such as peak overlap, peak integration, counting statistics, and peak-versus-background discrimination. Many of these errors are either cancelled or can be determined during experimental measurement of RIRs. For example, errors due to preferred orientation will be compensated for if the standards exhibit the same degree of preferred orientation as the samples to be analyzed. Errors due to peak integration and peak-versus-background discrimination are typicaliy similar for samples and standards and thus tend to be implicitly corrected for. However, errors may be significantly increased in our samples because of the presence of numerous phases with overlapping peaks. In these cases, errors due to partial or complete peak overlap, leading to problems in peak integration, are not explicitly accounted for. Therefore, we have incorporated a section of code into QUANT that can consider the presence of any combination of phases in the tuff samples and allow for the resulting peak overlaps when calculating relative errors. We consider both the errors in RIRs 
and the presence of overlapping pairs of phases when calculating errors. Included arriong the latter are the effect of the presence of (1) smectite on the relative errors for mordenite; (2) mordenite on clinoptiblite; (3) mordenite on feldspar; (4) feldspar on mordenite; (5) feldspar on cristobalite; (6) clinoptilolite on mordenite; (7) clinoptilolite on quartz; (8) clinoptilolite on feldspar; (9) tridymite on cristobalite; (10) tridymite on feldspar; and (11) opal-CT on clinoptilolite. The resulting relative errors are thus increased by every peak overlap for the sample, and we believe the reported errors for internal standard analyses are conservative. Errors for external standard analyses are typically larger than those inherent in internal standard analyses. The usual reason for employing only external standard analysis is insufficient sample, and in many cases amounts of sample available were too small to fill our standard sample holders.

\section{MINERAL DISTRIBUTIONS AT YUCCA MOUNTAIN}

The results of quantitative analyses are presented in tabular form in Appendix $A$ and, as noted above, supersede the tabular data presented in Bish and Vaniman (1985). We have not redrawn the geologic cross sections presented in Bish and Vaniman (1985) but instead have prepared figures illustrating the mineralogy as a function of depth in each drill hole for which we have sufficient data (Appendix B). The figures in Appendix B reveal many mineralogical details at a glance and illustrate some of the trends with depth discussed below. The mineral distributions at Yucca Mountain differ from drill hole to drill hole, but certain systematics are apparent. Bish and Vaniman (1985) described the distributions of each significant phase at Yucca Mountain. In this report we provide an updated summary of the mineralogy as a function of depth.

\section{Glass}

Glass, or material amorphous to $x$ rays, occurs above and below the potential repository host rock at Yucca Mountain (the welded, devitrified Topopah Spring Member, Tptw) but rarely occurs below the present-day static water level (SWL). The glasses can be divided into two categories: vitrophyric glass and vitric, nomwelded tuff. The vitrophyre is a zone of densely welded tuff at the base of the Topopah Spring Member that consists predominantly of glass with lesser amounts of smectite, feldspar, and silica minerals. Nonwelded vitric tuff occurs both above and below the Topopah Spring Member welded unit and is more abundant than vitrophyric glass across most of Yucca Mountain. The lower nonwelded vitric tuff thins and disappears to the east where the stratigraphic dip and structural displacements bring the basal glassy zone closer to the SWL. This vitric nonwelded material may have important paleohydrologic significance because the preservation of shards and pumice composed of nonwelded glass is rare below past SWLs. We have, however, identified glass below the water table in partially zeolitized material in USW G-3 1827.2 (556.9 m) below the water table. Preservation of glass in saturated rocks may be coupled to rock permeability or the "armoring" of glass by alteration phases. 


\section{Opal-CT}

As noted above, previous analyses of Yucca Mountain tutts did not distinguish opal-CT from cristobalite. Our most recent analyses include separate determinations of opal-CT, a disordered silica phase containing both cristobalite- and tridymite-like structural units (Jones and Segnit 1971). Almost without exception, Op:i-CT occurs in samples containing clinoptilolite andior mordenite. Thus the presence of opal-CT may be linked genetically to the formation of these two zeolites. Most of the opal-CT samples exhibit evidence for cristobalite-and tridymite-like stacking, but some more closely resemble opalC (predominantly cristobalite-like stacking).

\section{Silica Polymorphs}

The three crystalline sillca polymorphs, quartz, cristobalite, and tridymite, are abundant in the rocks at Yucca Mountain. Tridymite is common only in relatively shallow rocks, often associated with devitritication features and always above the SWL. It is absent in the north end of Yucca Mountain, where mineralogical evidence suggests that a high heat flow existed in the past (Bish and Semarge 1982). There appears to be a correlation between the loss of tridymite and the first appearance of abundant groundmass quartz with increasing depth. This transition in part reflects the passage from zones of common high-temperature vapor-phase crystallization (tridymite) to zones of lower-temperature devitrification (quartz) within the Topopah Spring Member.

In samples in which cristobalite was distinguished from opal-CT (se日 page 9), cristobalite is very restricted in rocks below the SWL, whereas it is present in virtually every shallow sample examined above 400-m depth. Because of overiap of the major cristobalite peak with significant feldspar peaks in $x$-ray diffraction patterns, the precision of analysis of cristobalite when presem in amounts below $10 \%$ is poor. Thus, the deeper samples identified as containing small amounts of cristobalite may in fact contain no cristobalite. The presence of cristobalite at shallow depths probably reflects the unsaturated nature of these rocks over much, if not all, of their history. Ernst and Cakven (1969) showed that an aqueous tuid is necessary for rapid recrystallization of cristobalite to quartz, and the recrystallization is accelerated in akkaline solutions. They concluded that conversion of the Monterey Formation porcelanite (cristobaime) to quartz in relatively pure water at $50^{\circ} \mathrm{C}$ would take between 4 and $5 \mathrm{~m} . \mathrm{y}$.

With depth, cristobalite gives way to opal-CT in zeolitized rocks in several drill holes. Quartz is present in most samples examined at Yucca Mountain, but it is typically less abundant at shallow depths and in glass-, clinoptilolite-, and mordenite-containing samples and generally increases in abundance below the depth where clinoptilolite and mordenite disappear, i.e., where cristobalite and opal-CT are no longer important silica phases.

\section{Smectite}

Smectite is present in small amounts in virtually all samples examined, but two zones of abundant smectite can be mapped across Yucca Mountain. These zones occur at the top of the vitric nomwelded base of the Tiva Canyon Member, which contains up to $35 \%$ smectite, and at the top of the basal 
vitrophyre of the Topopah Spring Member, which contains up to $45 \%$ smectite. The zones are generally less than $3 \mathrm{ft}(1 \mathrm{~m})$ thick but are thicker in drill holes LSW G-1, G-2, and UE-25a\#1. In addition, smectite concentrations are higher at all depths at the northern end of Yucca Mountain (USW G-1, USW G-2, UE$25 \mathrm{a} \# 1$, and UE-25b\#1), probably because of the higher heat flow in the past (Bish and Semarge 1982).

\section{Clinoptilolite/Heulandite and Mordenite}

Several studies have emphasized the occurrence of at least four distinct zones of clinoptiloliteheulandite $(\mathrm{C}-\mathrm{H})$ plus mordenite beneath Yucca Mountain (Bish et al. 1984; Vaniman et al. 1984!. These zones occur primarily where glassy material remained outside the zones of devitrification in the centers of ash flows. There is therefore a correlation between zeolitized material and the nonwelded tops, bottoms, and distal edges of ash flows. Exceptions to this correlation occur, including the zeolitized tuft in the devitrified Tram Member in USW G-3 and the zeolitized interval at the boundary between the devitrified zone and the vitrophyre of the Topopah Spring Member. In general, however, the thicker zeolitized horizons tend to correlate with those intervals that retained glass following early tuff devitrification.

Some chemical and crystallographic data suggest that the C.H zeolite above the basal vitrophyre in the Topopah Spring Member is heulandite, and similar data show that some deeper C-H zeolites coexisting with analcime are heulandite. We separated the $\mathrm{C}-\mathrm{H}$ from UE-25p\#1 at 3480-3490 $\mathrm{ft}$ (1060.7-1063.8 m) and performed heating experiments to discriminate between clinoptilolite and heulandite (Mumpton 1960). The material in this sample was clearly heulandite, as the diffraction pattern completely disappeared after heating to $450^{\circ} \mathrm{C}$ ovemight.

\section{Other Zeolites}

As reported by Bish and Vaniman (1985), none of the previous reports of erionite or phillipsite in Yucca Mountain rocks have been verified. However, Bish and Vaniman reported that one sample of erionite has been conclusively identitied in a fracture from UE-25a\#1 at $1296 \mathrm{ft}$ (395 m). Phillipsite has not yet been identified in any samples. We are currently surveying numerous Yucca Mountain samples for the presence of erionite using newly developed techniques that allow the detection of several hundred ppm erionite using $x$-ray powder diffraction (Bish and Chipera 1987). Chabazite was recently identified in a fracture sample in J-13 at 1345-ft (410-m) depth. Laumontite has been tentatively identitied in trace amounts near the bottom of USW G-1 and conclusively identified in four samples in UE-25p\#1. The occurrence of this $\mathrm{Ca}$-rich zeolite may reflect the influence of $\mathrm{Ca}$-rich groundwater on these rocks.

\section{Analcime and Albite}

Only a few drill holes have penetrated deep enough to intersect analcime-bearing zones and even fewer are deep enough to contain authigenic albite. In general, the shallowest occurrence of these two minerals is in the northern portion of Yucca Mountain, especially USW G-2, although analcime occurs in significant amounts in drill hole $\mathrm{J}-13$ at $1995-\mathrm{Ht}(608-\mathrm{m})$ depth. Mineralogic trends with depth suggest that anakime formed through alteration of clinoptilolite and that authigenic albite formed from anakime (Bish et al. 1981) 


\section{Economically important Minerals}

Although studies specifically directed at idemtifying occurrences of economically important minerals have not been performed by us to date, the results of our analyses allow some generalizations to be made. The most abundant minerals of potential economic importance at Yucca Mountain are the zeolites clinoptilolite and mordenite. These zeolites occur at Yucca Mountain in thick sequences comtaining up to $90 \%$ total 7.eolite. However, these deposits generally occur at depths greater than $1000 \mathrm{ft}$ (304 $\mathrm{m}$ ) (the shallowest occurrence is in USW G-2 at depths $>750 \mathrm{ft}$ ), making commercial utilization very difficult, expenstve, and therefore highly unlikely. Deposits of zeolite-rich rocks occur south of Yucca Mountain In a small outcrop at the base of Busted Butte and north at Prow Pass in a larger deposit. Both outcrops are of the tutf of Calico Hills and contain in excess of $50 \%$ total zeolite. Considering the presence of large, highpurity, near-surface deposits in Nevada, California, and Oregon and the low price of the natural zeolites clinoptilolite and mordenite, it is unlikely that the outcrop deposits near Yucca Mountain will become economically important unless a demand for clinoptilolite/mordenite-rich rock develops at the potential repository itself.

Other potentially economically important minerals at Yucca Mountain include suifides such as pyrite and cinnabar, fluorite, barite, gold, and sitver. Of these phases, only pyrite, fluorite, and barite have been identified by us at Yucca Moumtain. Fluorite occurs as a fracture or vein filling below 1000-tt depth in several drill holes and as a trace phase near the bottom of UE-25p\#1. Pyrite and bartie have both been identified optically near the Dottoms of some drill holes; even where optically identified, they are both present at levels below $1 \%$. Gold and siliver have not been idemified by either optical or $x$-ray diffraction methods, and chemical methods will be used to assess tiveir concentration in the rocks. Information to date provides no evidence for the presence of any economically important mirieral deposit at Yucca Mountain. For the potentially important phases present, low concentration and/or deep burial prechude economic use.

\section{SUMMARY}

Our quantitative analyses of Yucca Mountain rocks have improved significantly since the first analyses were conducted over nine years ago. We now routinely detect small amounts of glass, we are able to detect some phases below the percent level, and we can accommodate the variations in chemistry and preferred orientation common among many minerals in the Yucca Mountain rocks. In addition, we now routinely discriminate between opal-CT and cristobalite. Although many improvemems in technique have been made, our present results are still qualitatively similar to those first obtained by Heiken and Bevier (1979), Sykes et al. (1979), and Bish et al. (1981). Our $x$-ray diffraction methods are well suited to analysis of volcanic tutfs, rocks that in many cases are difficult to characterize completely by conventional optical methods. Quantitative mineralogic data on numerous drill holes at and around Yucca Mountain have alfowed us to understand the significant lateral and vertical mineralogic variations. A complete 
knowledge of the mineralogy existing at and around Yucca Mountain is importamt for us to be able to predict the minerabogy along possible flow paths away from the potential repository. In addition, understanding of the distribution of potentlally reactive phases such as zeolites, smectites, and glass will permit optimal siting of the potential repository and minimize possible deleterious interactions between the repository and the host rocks.

\section{ACKNOWLEGMENTS}

We appreciate a thorough review by $D$. Vaniman and comments by $S$. Mattson. We are grateful to M. Jones for typing the tables in Appendix A. 


\section{APPENDIX A}

X-PAY DIFFRACTION ANALYSES OF YUCCA MOUNTAIN TUFF FROM COAE, CUTTINGS, AND SIIEWALL SAMPLES 
APYENDIX A

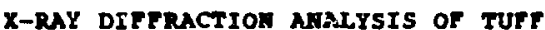

Cuttings

\begin{tabular}{|c|c|c|c|c|c|c|c|c|c|c|}
\hline sample & $\begin{array}{c}\text { Depth } \\
(=)\end{array}$ & suectite & Miea & $\begin{array}{l}\text { Clino- } \\
\text { ptilolite }\end{array}$ & $\begin{array}{l}\text { Tridy- } \\
\text { nite }\end{array}$ & Quartz & $\begin{array}{l}\text { Cristo- } \\
\text { balite }\end{array}$ & $\begin{array}{l}\text { Alkali } \\
\text { reldspar }\end{array}$ & Glass & Henatite \\
\hline \multicolumn{11}{|l|}{$J-12$} \\
\hline $620-630$ & $189.0-192.0$ & $9 \pm 4$ & $3 \pm 2$ & - & - & - & $6 \pm 3$ & $53 \pm 10$ & $30 \pm 20$ & - \\
\hline $650-660$ & $198.1-201.2$ & $<1$ & $2 \pm 2$ & $2 \pm 1$ & $23 \pm 4$ & $2 \pm 1$ & $10 \pm 3$ & $70 \pm 10$ & - & $\ll$ \\
\hline $110-720$ & $216.4-219.5$ & $<1$ & $1 \pm 1$ & - & $<2$ & $14 \pm 2$ & $7 \pm 3$ & $78 \pm 10$ & - & 1 \\
\hline $770-780$ & $234.7-237.7$ & $1 \pm 1$ & $=$ & - & - & $14 \pm 2$ & $4 \pm 2$ & $81 \pm 10$ & - & 1 \\
\hline $860-870$ & $262.1-265.2$ & 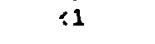 & - & - & - & $10 \pm 2$ & $4 \pm 2$ & $86 \pm 10$ & - & $1 \pm 1$ \\
\hline $905-915$ & $275.8-278.9$ & $3 \pm 2$ & < 1 & - & - & $12 \pm 2$ & $3 \pm 2$ & $83 \pm 10$ & - & a 1 \\
\hline $983-992$ & $299.6-302.4$ & e1 & (1) 1 & - & - & $24 \pm 3$ & \pm \pm 3 & $68 \pm 10$ & - & - \\
\hline $1067-1077$ & $325.2-328.3$ & <1 & (1) & - & - & $34 \pm 4$ & $3 \pm 2$ & $63 \pm 10$ & - & $<1$ \\
\hline $1093-1097$ & $333.1-334.4$ & $38 \pm 7$ & - & $51 \pm 10$ & - & $<1$ & $11 \pm 3$ & - & - & - \\
\hline $1107-1110$ & $337.4-334.3$ & $=$ & - & $4 \pm 2$ & - & $1 \pm 1$ & $6 \pm 3$ & $9 \pm 4$ & $80 \pm 20$ & - \\
\hline $1121-1126$ & $341.7-343.2$ & $3 \overline{3+7}$ & - & $\overline{5 s+10}$ & - & $2 \pm 1$ & $\begin{array}{r}4 \pm 2 \\
10+3\end{array}$ & $16 \pm 5$ & $80 \pm 20$ & - \\
\hline $1136-1139$ & $346.3-347.2$ & $33 \pm 7$ & - & $54 \pm 10$ & - & $2 \pm 1$ & $10 \pm 3$ & - & - & - \\
\hline
\end{tabular}


APPLNDIX A (cont)

core

\begin{tabular}{|c|c|c|c|c|c|c|c|c|c|c|c|c|c|c|c|c|}
\hline Sample" & $\begin{array}{l}\text { Depth } \\
\text { (a) }\end{array}$ & Method & snectite & Mica & $\begin{array}{l}\text { clino- } \\
\text { ptilolite }\end{array}$ & $\begin{array}{c}\text { Morden- } \\
\text { ite }\end{array}$ & $\begin{array}{l}\text { Ancl- } \\
\text { cies }\end{array}$ & $\begin{array}{l}\text { Iridy- } \\
\text { mite }\end{array}$ & Quartz & $\begin{array}{l}\text { Cristo- } \\
\text { balite }\end{array}$ & $\begin{array}{c}\text { Opal- } \\
\text { CT }\end{array}$ & $\begin{array}{l}\text { Alkali } \\
\text { reldepar }\end{array}$ & calcite & Glass & Benatite & other \\
\hline \multicolumn{17}{|l|}{$\mathrm{J}-13$} \\
\hline 427 & 130 & I & - & - & - & - & - & $15 \pm 1$ & Tr. & $14 \pm 1$ & - & $70 \pm 13$ & - & - & $T x$. & - \\
\hline 492 & 150 & I & - & - & - & - & - & $8 \pm 1$ & Tr. & $22 \pm 1$ & - & $67 \pm 10$ & - & - & - & - \\
\hline 591 & 180 & I & $5 \pm 1$ & - & - & - & - & - & $1 \pm 1$ & $=$ & - & - & - & $94 \pm 1$ & - & - \\
\hline 607 & 185 & $\mathbf{E}$ & $73 \pm 18$ & - & - & - & - & - & $\mathbf{T r}$. & $7 \pm 1$ & - & - & - & $20 \pm 18$ & - & - \\
\hline 689 & 210 & $\mathbf{E}$ & $26 \pm 5$ & - & $1 \pm 1$ & - & - & - & $5 \pm 1$ & $13 \pm 1$ & - & $54 \pm 6$ & - & - & $I r$. & - \\
\hline 801 & 244 & I & $2 \pm 1$ & - & - & - & - & $6 \pm 1$ & $1 \pm 1$ & $23 \pm 1$ & - & $66 \pm 10$ & - & - & Tr. & - \\
\hline 925 & 282 & I & $3 \pm 1$ & Tr. & - & - & - & $2 \pm 1$ & $29 \pm 1$ & $3 \pm 1$ & - & $62 \pm 8$ & - & - & - & - \\
\hline 1033 & 315 & I & $1 \pm 1$ & Tr. & - & - & $\Rightarrow$ & $2 \pm 1$ & $30 \pm 1$ & - & - & $69 \pm 9$ & - & - & $T x$. & - \\
\hline 1102 & 336 & I & $\mathbf{T r}$. & 25. & - & - & - & $2 \pm 1$ & $20 \pm 1$ & - & - & $78 \pm 10$ & - & - & Tr. & - \\
\hline 1194 & 364 & $\mathbf{E}$ & - & Tr. & - & - & - & $=$ & $33 \pm 1$ & - & - & $67 \pm 8$ & - & - & Tr. & - \\
\hline 1293 & 394 & I & - & Tr. & - & - & - & - & $33 \pm 1$ & - & - & $63 \pm 7$ & - & - & Tr. & - \\
\hline 1296 & 395 & $\mathbf{E}$ & - & - & - & - & - & - & $36 \pm 1$ & - & - & $64 \pm 8$ & - & - & Tr. & - \\
\hline 1345 & 110 & I & - & - & - & - & - & - & Tr. & - & $11 \pm 2$ & $1 \pm 1$ & - & $81_{ \pm 3}$ & - & - \\
\hline 1421 & 433 & $\boldsymbol{E}$ & $3 \pm 1$ & Tr. & $27 \pm 3$ & - & - & - & Ix. & - & $25 \pm 5$ & $13 \pm 2$ & - & $30 \pm 15$ & - & Tr. \\
\hline 1457 & 144 & I & $2 \pm 1$ & - & $80 \pm 8$ & - & - & - & $2 \pm 1$ & - & $8 \pm 2$ & $7 \pm 1$ & Tr. & - & - & - \\
\hline 1512 & 461 & I & $\mathbf{T r}$ & - & $17_{I 12}$ & - & - & - & $s_{ \pm 1}$ & - & $39 \pm 8$ & $6 \pm 1$ & - & - & - & - \\
\hline 1515 & 462 & I & $4 \pm 1$ & - & $64 \pm 6$ & - & - & - & $1 \pm 1$ & - & $12 \pm 2$ & $14 \pm 3$ & - & - & - & - \\
\hline 1519 & 163 & $I$ & $5 \pm 1$ & - & $56 \pm 8$ & $\mathbf{T r}$. & - & - & $7 \pm 1$ & - & $18 \pm 4$ & $13 \pm 2$ & - & - & - & - \\
\hline 1575 & 480 & I & $20 \pm 4$ & - & $=$ & - & - & - & $2 \pm 1$ & - & $=$ & $13 \pm 2$ & - & $65 \pm 5$ & - & - \\
\hline 1883 & 574 & $I$ & $1 \pm 1$ & Tr. & - & - & - & - & $34 \pm 1$ & - & - & $67 \pm 8$ & $\mathrm{Tr}$ & - & - & - \\
\hline 1995 & 608 & I & $2 \pm 1$ & - & - & - & $43 \pm 2$ & - & $33 \pm 1$ & - & - & $24 \pm 3$ & - & - & - & - \\
\hline 2001 & 610 & I & $2 \pm 1$ & Tr. & - & - & $12 \pm 2$ & - & $28 \pm 1$ & - & - & $29 \pm 3$ & - & - & - & - \\
\hline 2005 & 611 & I & $2 \pm 1$ & Tr. & - & - & $35 \pm 1$ & - & $27 \pm 1$ & - & - & $31 \pm 4$ & - & - & - & - \\
\hline 2133 & 650 & I & - & $2 \pm 1$ & - & - & $=$ & - & $32 \pm 1$ & - & - & $65 \pm 8$ & - & - & Tr. & - \\
\hline 2178 & 664 & $I$ & - & $1 \pm 1$ & - & - & - & - & $32 \pm 1$ & - & - & $66 \pm 8$ & Tr. & - & Tr. & - \\
\hline 2382 & 726 & I & $0 \pm 2$ & $3 \pm 1$ & - & - & $25 \pm 1$ & - & $34 \pm 1$ & - & - & $33 \pm 4$ & - & - & - & - \\
\hline 2533 & 772 & $\mathbf{I}$ & $2 \pm 1$ & $2 \pm 1$ & - & - & $\Rightarrow$ & - & $32 \pm 1$ & - & $\infty$ & $62 \pm 7$ & - & - & Tr. & - \\
\hline 2680 & 817 & $\mathbf{I}$ & $3 \pm 1$ & $3 \pm 1$ & - & - & - & - & $30 \pm 1$ & - & - & $61 \pm 7$ & Tr. & - & Tr. & - \\
\hline 2982 & 909 & $\mathbf{I}$ & $2 \pm 1$ & $2 \pm 1$ & - & - & - & - & $39 \pm 1$ & - & - & $56 \pm 7$ & Tr. & - & Tr. & - \\
\hline 2999 & 914 & I & $4 \pm 1$ & $1 \pm 1$ & - & - & $13 \pm 1$ & - & $36 \pm 1$ & - & - & $44 \pm 5$ & $2 \pm 1$ & - & - & $-d$ \\
\hline 3251 & 991 & $I$ & $33 \pm 7$ & Tr. & $2 \pm 1$ & - & $=$ & - & $21 \pm 1$ & - & - & $34 \pm 6$ & $7 \pm 1$ & - & - & $1 \pm 1^{d}$ \\
\hline
\end{tabular}

"sample designation, 0.g., 427 rofers to aseple frem drill hole J-13 at 427-ft dupth.

$b_{I}=$ Internal standard Mothod, $E$ - Extornal standard Mothod.

CHornblende.

dehlorite. 
Core

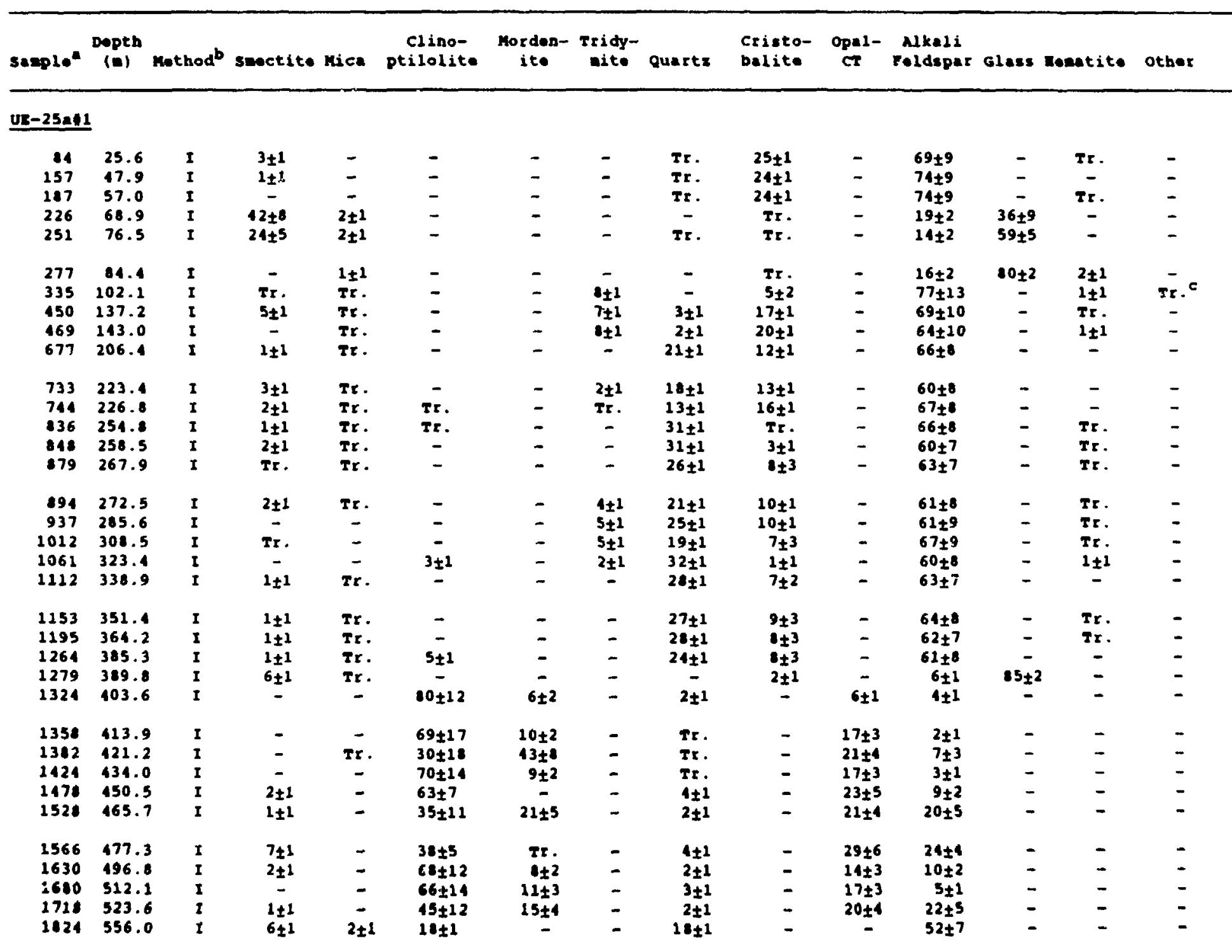


APPENDIX A (cont)

Core

\begin{tabular}{|c|c|c|c|c|c|c|c|c|c|c|c|c|c|c|}
\hline sanplea & $\begin{array}{c}\text { Depth } \\
\text { (I) }\end{array}$ & Mothod ${ }^{b}$ & smectite & Miea & $\begin{array}{l}\text { clino- } \\
\text { ptilolite }\end{array}$ & $\begin{array}{l}\text { Morden- } \\
\text { ite }\end{array}$ & $\begin{array}{l}\text { Tridy- } \\
\text { nite }\end{array}$ & Quarts & $\begin{array}{l}\text { Cristo- } \\
\text { belite }\end{array}$ & $\begin{array}{l}\text { Opa1- } \\
\text { CT }\end{array}$ & $\begin{array}{l}\text { Alkali } \\
\text { Peldspar }\end{array}$ & Glass & Hesatite & other \\
\hline \multicolumn{15}{|l|}{$u E-25 a 1$} \\
\hline 1852 & 564.5 & $\mathbf{I}$ & - & $T t$. & - & - & - & $41 \pm 1$ & - & - & $51 \pm 6$ & - & $1 \pm 1$ & $2 \pm 1 d$ \\
\hline 1904 & 580.3 & I & $7 \pm 1$ & Tr. & - & - & - & $34 \pm 1$ & - & - & $61 \pm 7$ & - & $T \tau$. & $\rightarrow$ \\
\hline 1953 & 595.3 & I & $2 \pm 1$ & Tr. & - & - & - & $33 \pm 1$ & - & - & $61 \pm 7$ & - & - & - \\
\hline 2002 & 610.2 & $\mathbf{E}$ & $\mathbf{T r}$. & Tr. & - & - & - & $11 \pm 1$ & - & - & $58 \pm 7$ & - & - & - \\
\hline 2051 & 625.1 & $\mathbf{I}$ & $5 \pm 1$ & - & - & - & - & $28 \pm 1$ & - & - & $65 \pm^{8}$ & - & $\rightarrow$ & - \\
\hline 2113 & 644.0 & $\mathbf{I}$ & $3 \pm 1$ & - & $11 \pm 1$ & - & - & $\mathbf{T r}$ & - & - & $17 \pm 2$ & $69 \pm 3$ & - & - \\
\hline 2220 & 676.7 & I & $1 \pm 1$ & - & $50 \pm 14$ & $18 \pm 5$ & - & $\mathbf{T r}$ & - & $15 \pm^{3}$ & $14 \pm 4$ & - & - & - \\
\hline 2304 & 702.3 & $I$ & $4 \pm 1$ & - & $39 \pm 7$ & $13 \pm 4$ & - & $12 \pm 1$ & - & - & $33 \pm 7$ & - & - & - \\
\hline 2361 & 719.6 & $I$ & $3 \pm 1$ & $1 \pm 1$ & - & - & - & $46 \pm 2$ & - & - & $50 \pm 6$ & - & $1 \pm 1$ & $\therefore d$ \\
\hline 2408 & 734.0 & $I$ & $2 \pm 1$ & $1 \pm 1$ & - & - & - & $38 \pm 1$ & - & - & $60 \pm 7$ & - & $\mathbf{T r}$ & $1 \pm 1^{d}$ \\
\hline 2449 & 746.5 & $I$ & $2 \pm 1$ & Tr. & - & - & - & $41 \pm 1$ & - & - & $58 \pm 7$ & - & Tr. & $1_{ \pm 1} d$ \\
\hline 2492 & 759.6 & I & $4 \pm 1$ & $1 \pm 1$ & - & - & - & $36 \pm 1$ & - & - & $57 \pm 7$ & - & Tr. & - \\
\hline
\end{tabular}

"sample designation, •.g., o4 refers to a saple frod drill hole uz-25all at o4-ft depth.

$b_{I}=$ Internal standard Mothod, $\varepsilon$ = External standard Mothod.

calcite.

dxaolinite. 
Core

\begin{tabular}{|c|c|c|c|c|c|c|c|c|c|c|c|}
\hline sanple & $\begin{array}{l}\text { Depth } \\
\text { (a) } 5\end{array}$ & smectite & MLea & $\begin{array}{l}\text { Clino- } \\
\text { ptilolite }\end{array}$ & $\begin{array}{l}\text { Morden- } \\
\text { ite }\end{array}$ & $\begin{array}{l}\text { Anal- } \\
\text { cine }\end{array}$ & guartz & $\begin{array}{l}\text { Alkali } \\
\text { eeldeper }\end{array}$ & calcite & anolinite & other \\
\hline \multicolumn{12}{|c|}{$U E-25 b 11 \mathrm{H}$} \\
\hline $\begin{array}{l}2402 \\
2450 \\
2525 \\
2596 \\
2651\end{array}$ & $\begin{array}{l}732.1 \\
746.8 \\
769.6 \\
791.3 \\
808.0\end{array}$ & $\begin{array}{l}15 \pm 5 \\
10 \pm 5 \\
10 \pm 5 \\
10 \pm 5 \\
10 \pm 5\end{array}$ & $\begin{array}{r}6 \pm 4 \\
7 \pm 3 \\
10 \pm 5 \\
6 \pm 4 \\
6 \pm 4\end{array}$ & $\begin{array}{l}- \\
- \\
- \\
-\end{array}$ & $\begin{array}{l}- \\
- \\
- \\
-\end{array}$ & $\begin{array}{l}- \\
- \\
-\end{array}$ & $\begin{array}{l}40 \pm 10 \\
40 \pm 10 \\
40 \pm 10 \\
40 \pm 10 \\
40 \pm 10\end{array}$ & $\begin{array}{l}40 \pm 10 \\
40 \pm 10 \\
40 \pm 10 \\
40 \pm 10 \\
40 \pm 10\end{array}$ & $\begin{array}{c}- \\
6 \pm 4 \\
6 \pm 4 \\
-\end{array}$ & $\begin{array}{l}- \\
- \\
-\end{array}$ & $\begin{array}{l}- \\
- \\
-\end{array}$ \\
\hline $\begin{array}{l}2737 \\
2832 \\
2847 \\
2855 \\
2867\end{array}$ & $\begin{array}{l}834.2 \\
863.2 \\
867.7 \\
870.2 \\
873.9\end{array}$ & $\begin{array}{r}3 \pm 2 \\
15 \pm 5 \\
7 \pm 3 \\
18 \pm 7 \\
18 \pm 7\end{array}$ & $\begin{array}{l}6 \pm 4 \\
6 \pm 4 \\
2 \pm 1 \\
3 \pm 2 \\
6 \pm 4\end{array}$ & $\begin{array}{l}- \\
3 \pm 2 \\
62 \\
3 \pm 2 \\
-\end{array}$ & $\begin{array}{r}- \\
18 \pm 7 \\
18 \pm 9 \\
18 \pm 7 \\
3 \pm 2\end{array}$ & $\begin{array}{l}- \\
\overline{-} \\
\bar{z}\end{array}$ & $\begin{array}{l}30 \pm 10 \\
23 \pm 7 \\
29 \pm 4 \\
23 \pm 7 \\
30 \pm 10\end{array}$ & $\begin{array}{l}50 \pm 10 \\
30 \pm 10 \\
44 \pm 10 \\
30 \pm 10 \\
50 \pm 10\end{array}$ & $\begin{array}{c}- \\
3 \pm 2 \\
\overline{6 \pm 4} \\
-\end{array}$ & $\begin{array}{l}- \\
- \\
- \\
-\end{array}$ & $\begin{array}{l}- \\
- \\
-\end{array}$ \\
\hline $\begin{array}{l}2879 \\
2919 \\
2946 \\
2953 \\
2988\end{array}$ & $\begin{array}{l}877.5 \\
889.7 \\
897.9 \\
900.1 \\
910.7\end{array}$ & $\begin{array}{c}23 \pm 7 \\
- \\
6 \pm 4 \\
6 \pm 4 \\
10 \pm 5\end{array}$ & $\begin{array}{r}15 \pm 5 \\
10 \pm 5 \\
6 \pm 4 \\
6 \pm 4 \\
10 \pm 5\end{array}$ & $\begin{array}{c}10 \pm 5 \\
= \\
= \\
= \\
z\end{array}$ & $\begin{array}{c}10 \pm 5 \\
30 \pm 10 \\
= \\
- \\
=\end{array}$ & $\begin{array}{l}- \\
= \\
-\end{array}$ & $\begin{array}{l}30 \pm 10 \\
23 \pm 7 \\
40 \pm 10 \\
40 \pm 10 \\
40 \pm 10\end{array}$ & $\begin{array}{l}30 \pm 10 \\
30 \pm 10 \\
40 \pm 10 \\
40 \pm 10 \\
10 \pm 10\end{array}$ & $\begin{array}{c}6 \pm 4 \\
- \\
6 \pm 4 \\
6 \pm 4 \\
3 \pm 2\end{array}$ & $\begin{array}{l}= \\
= \\
- \\
-\end{array}$ & $\begin{array}{l}- \\
- \\
- \\
-\end{array}$ \\
\hline $\begin{array}{l}3050 \\
3092 \\
3095 \\
3098\end{array}$ & $\begin{array}{r}929.6 \\
942.4 \\
\text { Frecture } \\
943.4 \\
944.3\end{array}$ & $\begin{array}{c}10 \pm 5 \\
6 \pm 4 \\
- \\
3 \pm 2 \\
10 \pm 5\end{array}$ & $\begin{array}{r}10 \pm 5 \\
7 \pm 3 \\
6 \pm 4 \\
10 \pm 5 \\
10 \pm 5\end{array}$ & $\begin{array}{l}3 \pm 2 \\
\mathbf{T r} \\
- \\
- \\
-\end{array}$ & $\begin{array}{l}- \\
- \\
-\end{array}$ & $\begin{array}{l}- \\
- \\
-\end{array}$ & $\begin{array}{l}30 \pm 10 \\
50 \pm 10 \\
40 \pm 10 \\
40 \pm 10 \\
30 \pm 10\end{array}$ & $\begin{array}{l}50 \pm 10 \\
10 \pm 10 \\
30 \pm 10 \\
50 \pm 10 \\
50 \pm 10\end{array}$ & $\begin{array}{r}6 \pm 4 \\
3 \pm 2 \\
23 \pm 7 \\
- \\
3 \pm 2\end{array}$ & $\begin{array}{l}- \\
- \\
-\end{array}$ & $\begin{array}{l}- \\
- \\
- \\
-\end{array}$ \\
\hline $\begin{array}{l}3128 \\
3163 \\
3183\end{array}$ & $\begin{array}{r}953.4 \\
\text { Practure } \\
964.1 \\
\text { practure } \\
970.8 \\
\text { practure }\end{array}$ & $\begin{array}{r}10 \pm 5 \\
3 \pm 2 \\
3 \pm 2 \\
- \\
18 \pm 7 \\
3 \pm 2\end{array}$ & $\begin{array}{r}10 \pm 5 \\
3 \pm 2 \\
10 \pm 5 \\
3 \pm 2 \\
13 \pm 5 \\
3 \pm 2\end{array}$ & $\begin{array}{l}= \\
= \\
= \\
=\end{array}$ & $\begin{array}{l}= \\
= \\
= \\
-\end{array}$ & $\begin{array}{l}- \\
\overline{-} \\
- \\
-\end{array}$ & $\begin{array}{l}30 \pm 10 \\
30 \pm 10 \\
30 \pm 10 \\
20 \pm 10 \\
30 \pm 10 \\
10 \pm 5\end{array}$ & $\begin{array}{l}50 \pm 10 \\
30 \pm 10 \\
50 \pm 10 \\
20 \pm 10 \\
10 \pm 10\end{array}$ & $\begin{array}{c}3 \pm 2 \\
23 \pm 7 \\
3 \pm 2 \\
60 \pm 10 \\
- \\
70 \pm 10\end{array}$ & $\begin{array}{l}- \\
\overline{-} \\
- \\
-\end{array}$ & 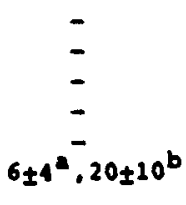 \\
\hline $\begin{array}{l}3196 \\
3222 \\
3225 \\
3257 \\
3267\end{array}$ & $\begin{array}{l}974.1 \\
982.1 \\
943.0 \\
992.7 \\
995.8\end{array}$ & $\begin{array}{c}6 \pm 4 \\
3 \pm 2 \\
10 \pm 5 \\
10 \pm 10 \\
23 \pm 7\end{array}$ & $\begin{array}{r}10 \pm 5 \\
10 \pm 5 \\
10 \pm 5 \\
10 \pm 5 \\
6 \pm 4\end{array}$ & $\begin{array}{l}- \\
- \\
- \\
-\end{array}$ & $\begin{array}{l}- \\
= \\
-\end{array}$ & $\begin{array}{l}- \\
- \\
-\end{array}$ & $\begin{array}{l}30 \pm 10 \\
30 \pm 10 \\
30 \pm 10 \\
20 \pm 10 \\
40 \pm 10\end{array}$ & $\begin{array}{l}50 \pm 10 \\
60 \pm 10 \\
50 \pm 10 \\
30 \pm 10 \\
30 \pm 10\end{array}$ & $\begin{array}{l}- \\
= \\
=\end{array}$ & $\begin{array}{l}- \\
= \\
-\end{array}$ & $\begin{array}{l}- \\
\overline{-} \\
\overline{-}\end{array}$ \\
\hline $\begin{array}{l}3298 \\
3326 \\
3362 \\
3374 \\
3393\end{array}$ & $\begin{array}{l}1005.2 \\
1013.8 \\
1024.7 \\
1026.4 \\
1034.2\end{array}$ & $\begin{array}{l}10 \pm 5 \\
10 \pm 5 \\
18 \pm 7 \\
10 \pm 5 \\
10 \pm 5\end{array}$ & $\begin{array}{r}6 \pm 4 \\
10 \pm 5 \\
10 \pm 5 \\
10 \pm 5 \\
7 \pm 3\end{array}$ & $\begin{array}{l}- \\
- \\
-\end{array}$ & $\begin{array}{l}- \\
- \\
=\end{array}$ & $\begin{array}{c}\mathrm{Tr} \\
3 \pm 2 \\
3 \pm 2 \\
20 \pm 10 \\
20 \pm 10\end{array}$ & $\begin{array}{l}30 \pm 10 \\
30 \pm 10 \\
30 \pm 10 \\
30 \pm 10 \\
30 \pm 10\end{array}$ & $\begin{array}{l}50 \pm 10 \\
50 \pm 10 \\
40 \pm 10 \\
30 \pm 10 \\
30 \pm 10\end{array}$ & $\begin{array}{c}\overline{-} \\
\overline{-} \\
10 \pm 5\end{array}$ & $\begin{array}{c}- \\
- \\
\mathrm{Tr} \\
2 \\
3 \pm 2\end{array}$ & $\begin{array}{l}- \\
- \\
- \\
-\end{array}$ \\
\hline
\end{tabular}


APPENDIX A (continued)

Core

\begin{tabular}{|c|c|c|c|c|c|c|c|c|c|c|c|}
\hline Sample & $\begin{array}{l}\text { Depth } \\
\text { (a) si }\end{array}$ & smectite & Mica & $\begin{array}{c}\text { clino- } \\
\text { ptilolite }\end{array}$ & $\begin{array}{l}\text { Morden- } \\
\text { ite }\end{array}$ & $\begin{array}{l}\text { Anal- } \\
\text { ci=e }\end{array}$ & Quarts & $\begin{array}{c}\text { Alkeli } \\
\text { reldspar }\end{array}$ & Calcite & Kaolinite & other \\
\hline \multicolumn{12}{|c|}{$U E-25 b \| 1 H$} \\
\hline $\begin{array}{l}3401 \\
3459 \\
3469 \\
3506 \\
3530\end{array}$ & $\begin{array}{r}1036.6 \\
1054.3 \\
1057.4 \\
1068.6 \\
1075.9 \\
\text { Practure } \\
\end{array}$ & $\begin{array}{r}3 \pm 2 \\
10 \pm 5 \\
10 \pm 5 \\
18 \pm 7 \\
15 \pm 5 \\
15 \pm 5\end{array}$ & $\begin{array}{r}6 \pm 4 \\
10 \pm 5 \\
10 \pm 5 \\
10 \pm 5 \\
7 \pm 3 \\
-\end{array}$ & $\begin{array}{l}\overline{-} \\
\bar{z} \\
-\end{array}$ & $\begin{array}{l}- \\
- \\
- \\
-\end{array}$ & $\begin{array}{l}20 \pm 10 \\
20 \pm 10 \\
20 \pm 10 \\
20 \pm 10 \\
23 \pm 7 \\
10 \pm 5\end{array}$ & $\begin{array}{l}30 \pm 10 \\
30 \pm 10 \\
30 \pm 10 \\
30 \pm 10 \\
30 \pm 10 \\
30 \pm 10\end{array}$ & $\begin{array}{l}30 \pm 10 \\
23 \pm 7 \\
30 \pm 10 \\
30 \pm 10 \\
40 \pm 10 \\
30 \pm 10\end{array}$ & $\begin{array}{l}3 \pm 2 \\
6 \pm 4 \\
6 \pm 4 \\
6 \pm 4 \\
3 \pm 2 \\
=\end{array}$ & $\begin{array}{c}3 \pm 2 \\
6 \pm 4 \\
6 \pm \\
6 \pm 4 \\
<2 \\
7 \pm 3\end{array}$ & $\begin{array}{l}\overline{-} \\
\overline{-} \\
\overline{-}\end{array}$ \\
\hline $\begin{array}{l}3548 \\
3571 \\
3572\end{array}$ & $\begin{array}{r}1081.4 \\
\text { Inclusion } \\
1088.4 \\
1088.7 \\
\text { Inclusion }\end{array}$ & $\begin{array}{r}10 \pm 5 \\
15 \pm 5 \\
7 \pm 3 \\
6 \pm 4 \\
18 \pm ?\end{array}$ & $\begin{array}{r}10 \pm 5 \\
10 \pm 5 \\
6 \pm 4 \\
6 \pm 4 \\
10 \pm 5\end{array}$ & $\begin{array}{l}= \\
=\end{array}$ & $\begin{array}{l}- \\
- \\
-\end{array}$ & $\begin{array}{c}3 \pm 2 \\
- \\
3 \pm 2 \\
<2 \\
-\end{array}$ & $\begin{array}{l}30 \pm 10 \\
23 \pm 7 \\
10 \pm 10 \\
30 \pm 10 \\
30 \pm 10\end{array}$ & $\begin{array}{l}40 \pm 10 \\
40 \pm 10 \\
23 \pm 7 \\
40 \pm 10 \\
30 \pm 10\end{array}$ & $\begin{array}{l}6 \pm 4 \\
15 \pm 5 \\
30 \pm 10 \\
23 \pm 7 \\
10 \pm 5\end{array}$ & $\begin{array}{l}3 \pm 2 \\
<2 \\
3 \pm 2 \\
<2 \\
<2\end{array}$ & $\begin{array}{l}- \\
- \\
-\end{array}$ \\
\hline $\begin{array}{l}3602 \\
3660 \\
3708\end{array}$ & $\begin{array}{r}1097.9 \\
\text { recture } \\
1115.6 \\
\text { Practure } \\
1130.2\end{array}$ & $\begin{array}{r}10 \pm 5 \\
3 \pm 2 \\
10 \pm 5 \\
- \\
10 \pm 5\end{array}$ & $\begin{array}{c}6 \pm 4 \\
- \\
6 \pm 4 \\
- \\
6 \pm 4\end{array}$ & $\begin{array}{l}- \\
-\end{array}$ & $\begin{array}{l}- \\
- \\
-\end{array}$ & $\begin{array}{l}20 \pm 10 \\
10 \pm 5 \\
23 \pm 7 \\
23 \pm 7 \\
23 \pm 7\end{array}$ & $\begin{array}{c}30 \pm 10 \\
6 \pm 4 \\
30 \pm 10 \\
15 \pm 5 \\
30 \pm 10\end{array}$ & $\begin{array}{c}30 \pm 10 \\
- \\
30 \pm 10 \\
6 \pm 4 \\
40 \pm 10\end{array}$ & $\begin{array}{c}10 \pm 5 \\
40 \pm 10 \\
6 \pm 1 \\
60 \pm 10 \\
=\end{array}$ & $\begin{array}{c}2 \\
3 \pm 2 \\
3 \pm 2\end{array}$ & $\begin{array}{c}- \\
40 \pm 10^{\circ} \\
- \\
- \\
-\end{array}$ \\
\hline $\begin{array}{l}3767 \\
3792 \\
3335 \\
3880 \\
3901\end{array}$ & $\begin{array}{l}1148.2 \\
1155.8 \\
1168.9 \\
1182.6 \\
1189.0\end{array}$ & $\begin{array}{l}10 \pm 5 \\
15 \pm 5 \\
15 \pm 5 \\
15 \pm 5 \\
10 \pm 10\end{array}$ & $\begin{array}{r}10 \pm 5 \\
7 \pm 3 \\
7 \pm 3 \\
7 \pm 3 \\
6 \pm 4\end{array}$ & $\begin{array}{l}- \\
- \\
-\end{array}$ & $\begin{array}{l}- \\
- \\
- \\
-\end{array}$ & $\begin{array}{c}23 \pm 7 \\
10 \pm 5 \\
23 \pm 7 \\
15 \pm 5 \\
7 x .\end{array}$ & $\begin{array}{l}35 \pm 10 \\
30 \pm 10 \\
30 \pm 10 \\
30 \pm 10 \\
30 \pm 10\end{array}$ & $\begin{array}{l}23 \pm 7 \\
40 \pm 10 \\
30 \pm 10 \\
30 \pm 10 \\
23 \pm 7\end{array}$ & $\begin{array}{r}10 \pm 5 \\
6 \pm 4 \\
6 \pm 4 \\
10 \pm 5 \\
-\end{array}$ & $\begin{array}{l}6 \pm 4 \\
3 \pm 2 \\
3 \pm 2 \\
3 \pm 2 \\
T r .\end{array}$ & $\begin{array}{l}- \\
- \\
-\end{array}$ \\
\hline $\begin{array}{l}3902 \\
3904 \\
3910 \\
3926 \\
3956\end{array}$ & $\begin{array}{l}1189.3 \\
1189.9 \\
1191.8 \\
1196.6 \\
1205.8\end{array}$ & $\begin{array}{l}10 \pm 10 \\
30 \pm 10 \\
10 \pm 5 \\
15 \pm 5 \\
23 \pm 7\end{array}$ & $\begin{array}{c}3 \pm 2 \\
- \\
- \\
10 \pm 5 \\
10 \pm 5\end{array}$ & $\begin{array}{l}- \\
\overline{-} \\
-\end{array}$ & $\begin{array}{c}6 \pm 4 \\
- \\
- \\
-\end{array}$ & $\begin{array}{c}- \\
23 \pm 7 \\
6 \pm 4 \\
\overline{3} \\
3 \pm 2\end{array}$ & $\begin{array}{l}23 \pm 7 \\
30 \pm 10 \\
40 \pm 10 \\
30 \pm 10 \\
23 \pm 7\end{array}$ & $\begin{array}{l}23 \pm 7 \\
23 \pm 7 \\
40 \pm 20 \\
30 \pm 10 \\
40 \pm 10\end{array}$ & $\begin{array}{r}23 \pm 7 \\
3 \pm 2 \\
- \\
15 \pm 5 \\
6 \pm 4\end{array}$ & $\begin{array}{c}- \\
- \\
6 \pm 4 \\
3 \pm 2\end{array}$ & $\begin{array}{l}- \\
- \\
- \\
-\end{array}$ \\
\hline $\begin{array}{l}3963 \\
3988\end{array}$ & $\begin{array}{r}1207.9 \\
1215.5 \\
\text { rracture } \\
\end{array}$ & $\begin{array}{c}30 \pm 10 \\
18 \pm 7 \\
3 \pm 2\end{array}$ & $\begin{array}{r}10 \pm 5 \\
18 \pm 7 \\
3 \pm 2\end{array}$ & $\bar{z}$ & $\begin{array}{l}- \\
-\end{array}$ & $\begin{array}{c}18 \pm 7 \\
- \\
-\end{array}$ & $\begin{array}{l}30 \pm 10 \\
30 \pm 10 \\
10 \pm 5\end{array}$ & $\begin{array}{l}30 \pm 10 \\
40 \pm 10 \\
15 \pm 5\end{array}$ & $\begin{array}{c}6 \pm 4 \\
- \\
80 \pm 10\end{array}$ & $\begin{array}{l}\text { Tr. } \\
- \\
-\end{array}$ & $\begin{array}{l}\overline{-} \\
\overline{-}\end{array}$ \\
\hline
\end{tabular}




\begin{tabular}{|c|c|c|c|c|c|c|c|c|c|c|c|c|c|c|c|c|}
\hline sanploa & $\begin{array}{c}\text { Depth } \\
\text { (a) }\end{array}$ & Mothod ${ }^{\mathbf{b}}$ & seoctite & Mica & $\begin{array}{l}\text { Clino- } \\
\text { ptilolite }\end{array}$ & $\begin{array}{l}\text { Morden- } \\
\text { ite }\end{array}$ & $\begin{array}{l}\operatorname{Anal}-I \\
\text { cine }\end{array}$ & $\begin{array}{l}\text { Tridy- } \\
\text { nite }\end{array}$ & Quarte & $\begin{array}{l}\text { Cristo- } \\
\text { balito }\end{array}$ & $\begin{array}{c}\text { Opal- } \\
\text { CT }\end{array}$ & $\begin{array}{c}\text { Alkali } \\
\text { reldspar }\end{array}$ & $\begin{array}{l}\text { Hean- } \\
\text { tite }\end{array}$ & $\begin{array}{l}\text { Cal- } \\
\text { cite }\end{array}$ & $\begin{array}{l}\text { Dolo- } \\
\text { oite }\end{array}$ & Other \\
\hline \multicolumn{17}{|l|}{ UE-25p:1 } \\
\hline $200-210$ & $61.8-64.0$ & $\mathbf{I}$ & - & $1 \pm 1$ & - & - & - & $14 \pm 1$ & - & $6 \pm 3$ & - & $76 \pm 14$ & $2 \pm 2$ & - & - & - \\
\hline $260-270$ & $79.2-82.3$ & $\mathbf{E}$ & $2 \pm 1$ & $r \bar{r}$. & - & - & - & $\mathbf{T r}$ & $3 \pm 1$ & $24 \pm 4$ & - & $71 \pm 14$ & - & - & - & - \\
\hline $280-290$ & $85.3-88.4$ & $\mathbf{E}$ & $2 \pm 1$ & $\mathbf{T r}$ & - & - & - & $\mathbf{T r}$ & $5 \pm 1$ & $25 \pm 4$ & - & $68 \pm 14$ & - & - & - & - \\
\hline $110-420$ & $125.0-128.0$ & $\mathbf{I}$ & $3 \pm 1$ & $\mathbf{T r}$ & - & - & - & $1 \pm 1$ & $9 \pm 1$ & $24 \pm 2$ & - & $59 \pm 8$ & $1 \pm 1$ & - & - & - \\
\hline $570-580$ & $173.7-176.8$ & $\mathbf{I}$ & $2 \pm 1$ & $\mathbf{T r}$ & - & - & - & $T r$ & $30 \pm 2$ & $3 \pm 1$ & - & $67 \pm 8$ & $\mathbf{T r}$ & - & - & - \\
\hline $210-820$ & $246.9-249.9$ & I & $2 \pm 1$ & Tr. & - & - & - & - & $27 \pm 2$ & $5 \pm 2$ & - & $59 \pm 7$ & $\mathbf{T r}$ & - & - & - \\
\hline $900-910$ & $274.3-277.4$ & I & $1 \pm 1$ & $\mathbf{T r}$. & - & - & - & - & $36 \pm 2$ & $1 \pm 1$ & - & $61 \pm 7$ & Tr. & - & - & - \\
\hline $1040-1050$ & $317.0-320.0$ & $I$ & - & $\mathbf{T r}$ & - & - & - & - & $27 \pm 2$ & $5 \pm 2$ & - & $67 \pm 8$ & $\mathbf{T r}$ & - & - & - \\
\hline $1240-1250$ & $378.0-381.0$ & $E$ & $1 \pm 1$ & - & $54 \pm 8$ & - & - & - & $\mathbf{T} x$ & - & $20 \pm 8$ & $24 \pm 0^{\circ}$ & - & - & - & - \\
\hline $1260-1270$ & $384.0-387.1$ & $\boldsymbol{E}$ & $1 \pm 1$ & - & $62 \pm 10$ & - & - & - & $\mathbf{T r}$ & - & $23 \pm 8$ & $14 \pm 4$ & - & - & - & - \\
\hline $1290 \mathrm{SW}$ & 393.2 & I & $2 \pm 1$ & - & $37 \pm 3$ & - & - & - & $3 \pm 1$ & - & $22 \pm 9$ & $39 \pm 6$ & - & - & - & - \\
\hline $1340-1350$ & $408.4-411.5$ & I & $\mathbf{T r}$ & - & $56 \pm 4$ & - & - & - & $2 \pm 1$ & - & $20 \pm 6$ & $21 \pm 4$ & - & - & - & - \\
\hline $14005 w$ & 426.7 & $I$ & $10 \pm 3$ & $1 \pm 1$ & $34 \pm 2$ & - & - & - & $17 \pm 1$ & - & - & $29 \pm 4$ & - & - & - & $9 \pm 6^{c}$ \\
\hline $14205 W$ & 432.8 & I & $12 \pm 4$ & $1 \pm 1$ & - & - & - & - & $24 \pm 2$ & - & - & $40 \pm 5$ & $1 \pm 1$ & - & - & $22 \pm 7^{c}$ \\
\hline $14705 w$ & 448.1 & I & $5 \pm 2$ & - & - & $34 \pm 11$ & - & - & $25 \pm 2$ & - & - & $40 \pm 12$ & $=$ & - & - & $2 \pm 1^{d}$ \\
\hline $1590-1598$ & $484.6-487.1$ & $I$ & - & - & $3 \pm 1$ & - & - & - & $39 \pm 2$ & - & - & $60 \pm 7$ & $1 \pm 1$ & - & - & - \\
\hline $1640-1650$ & $499.9-502.9$ & 1 & $5 \pm 2$ & - & - & - & - & - & $37 \pm 2$ & - & - & $58 \pm 7$ & $1 \pm 1$ & - & - & - \\
\hline $1690-1700$ & $515.1-518.2$ & I & $7 \pm 2$ & - & $40 \pm 3$ & - & - & - & $11 \pm 1$ & - & $17 \pm 6$ & $25 \pm 4$ & - & - & - & - \\
\hline $1730-1740$ & $527.3-530.4$ & I & $3 \pm 1$ & - & $60 \pm 4$ & - & - & - & $4 \pm 1$ & - & $14 \pm 4$ & $17 \pm 3$ & - & - & - & - \\
\hline $1790-1800$ & $545.6-548.6$ & I & $1 \pm 1$ & - & $45 \pm 8$ & $23 \pm 7$ & - & - & $12 \pm 1$ & - & - & $18 \pm 5$ & - & - & - & - \\
\hline $1830-1840$ & $557.8-560.8$ & I & $\mathrm{Tr}$ & $2 \pm 1$ & - & - & - & - & $45 \pm 2$ & - & - & $39 \pm 5$ & $1 \pm 1$ & - & - & $12 \pm 3^{d}$ \\
\hline $1870-1880$ & $570.0-573.0$ & I & $3 \pm 1$ & $=$ & $9 \pm 1$ & - & - & - & $27 \pm 2$ & - & - & $55 \pm 7$ & $\mathbf{T r}$. & - & - & - \\
\hline $1920-1930$ & $585.2-588.3$ & 1 & $5 \pm 2$ & $2 \pm 1$ & $=$ & - & - & - & $28 \pm 2$ & - & - & $64 \pm 8$ & $2 \pm 2$ & - & - & - \\
\hline $1970-1980$ & $600.5-603.5$ & 1 & $\mathbf{T r}$. & $1 \pm 1$ & - & - & - & - & $35 \pm 2$ & - & $\sim$ & $61 \pm 7$ & $1 \pm 1$ & - & - & - \\
\hline $1990-2000$ & $606.6-609.6$ & I & $1 \pm 1$ & $1 \pm 1$ & - & - & - & - & $39 \pm 2$ & - & - & $60 \pm 7$ & $\mathbf{T r}$ & - & - & - \\
\hline $2030-2040$ & $618.7-621.8$ & 1 & $2 \pm 1$ & Tr. & - & - & - & - & $31 \pm 2$ & - & - & $59 \pm 7$ & $1 \pm 1$ & - & - & $7 \pm 7^{c}$ \\
\hline $2070-2080$ & $630.9-634.0$ & I & $\mathbf{r} \mathbf{r}$ & Tr. & - & - & - & - & $39 \pm 2$ & - & - & $59 \pm 7$ & $1 \pm 1$ & - & - & - \\
\hline $2120-2130$ & $646.2-649.2$ & $\mathbf{I}$ & $\mathbf{T r}$ & Tr. & - & - & - & - & $36 \pm 2$ & - & - & $65 \pm 8$ & $1 \pm 1$ & - & - & - \\
\hline $2150-2160$ & $655.3-658.4$ & I & $3 \pm 1$ & Tr. & $\mathbf{T r}$ & - & - & - & $29 \pm 2$ & - & - & $61 \pm 7$ & $1 \pm 1$ & $1 \pm 1$ & $\cdots$ & - \\
\hline $2210-2220$ & $673.6-676.7$ & $I$ & $3 \pm 1$ & - & - & $28 \pm 8$ & $14 \pm 1$ & - & $28 \pm 2$ & - & - & $33 \pm 9$ & - & $T r$ & - & - \\
\hline $2240-2250$ & $682.8-685.8$ & $I$ & $7 \pm 2$ & $1 \pm 1$ & - & $3 \pm 1$ & $8 \pm 1$ & - & $29 \pm 2$ & - & - & $52 \pm 7$ & - & - & - & - \\
\hline $2280-2290$ & $694.9-698.0$ & $\mathbf{I}$ & $6 \pm 2$ & $3 \pm 1$ & $31 \pm 2$ & - & $4 \pm 1$ & - & $22 \pm 2$ & - & - & $32 \pm 5$ & - & Tr. & - & - \\
\hline $2330-2340$ & $710.2-713.2$ & $I$ & $11 \pm 3$ & $1 \pm 1$ & $2 \pm 1$ & - & $1 \pm 1$ & - & $32 \pm 2$ & - & - & $45 \pm 5$ & - & $\mathbf{T r}$. & - & - \\
\hline 2370-2380 & $722 \cdot 4-725.4$ & $I$ & $7 \pm 2$ & $2 \pm 1$ & - & - & - & - & $40 \pm 2$ & - & - & $49 \pm 6$ & $1 \pm 1$ & - & - & - \\
\hline $2410-2420$ & $734.6-737.6$ & I & Tr. & $1 \pm 1$ & - & - & - & - & $43 \pm 2$ & - & - & $51 \pm 6$ & $1 \pm 1$ & - & - & Tr. \\
\hline
\end{tabular}


APPEKOIX A (cont)

cuttings, sidewall, and core

\begin{tabular}{|c|c|c|c|c|c|c|c|c|c|c|c|c|c|c|c|c|}
\hline Sanplea & $\begin{array}{c}\text { Depth } \\
(\text { m) }\end{array}$ & lethod & suectite & Mica & $\begin{array}{l}\text { Clino- } \\
\text { ptilolite }\end{array}$ & $\begin{array}{l}\text { Morden- } \\
\text { ite }\end{array}$ & $\begin{array}{l}\text { Anal- } \\
\text { cime }\end{array}$ & $\begin{array}{l}\text { Tridy- } \\
\text { vite }\end{array}$ & guartz & $\begin{array}{l}\text { Cristo- } \\
\text { balite }\end{array}$ & $\begin{array}{l}\text { Opal- } \\
\text { CT }\end{array}$ & $\begin{array}{l}\text { Alkali } \\
\text { Feldspar }\end{array}$ & $\begin{array}{l}\text { Hena- } \\
\text { tite }\end{array}$ & $\begin{array}{l}\text { Cal- } \\
\text { cite }\end{array}$ & $\begin{array}{l}\text { Do!o- } \\
\text { mite }\end{array}$ & other \\
\hline \multicolumn{17}{|l|}{ UE-25p\$1 } \\
\hline $2460-2470$ & $749.8-752.9$ & I & $2 \pm 1$ & $1 \pm 1$ & - & - & - & - & $32 \pm 2$ & - & - & $61 \pm 7$ & $1 \pm 1$ & - & - & $1 \pm 1^{d}$ \\
\hline $2510-2520$ & $765.0-768.1$ & I & - & $2 \pm 1$ & - & - & - & - & $24 \pm 2$ & - & - & $77 \pm 9$ & $1 \pm 1$ & - & - & $=$ \\
\hline $2570-2580$ & $783.3-786.4$ & I & $2 \pm 1$ & $2 \pm 1$ & - & - & - & - & $30 \pm 2$ & - & - & $71 \pm 8$ & $1 \pm 1$ & - & - & - \\
\hline $2630-2640$ & $801.6-804.7$ & $I$ & $7 \pm 2$ & $1 \pm 1$ & - & - & - & - & $34 \pm 2$ & - & - & $57 \pm 7$ & $1 \pm 1$ & - & - & - \\
\hline $2650-2660$ & $807.7-810.8$ & $I$ & $15 \pm 5$ & $1 \pm 1$ & - & - & - & - & $31 \pm 2$ & - & - & $51 \pm 6$ & $2 \pm 1$ & $1 \pm 1$ & - & - \\
\hline $2690-2700$ & $819.9-823.0$ & $I$ & $4 \pm 1$ & $1 \pm 1$ & - & - & - & - & $34 \pm 2$ & - & - & $56 \pm 7$ & $1 \pm 1$ & $\mathbf{T r}$. & - & - \\
\hline $2750-2760$ & $838.2-841.2$ & I & $13 \pm 4$ & $1 \pm 1$ & $13 \pm 1$ & - & - & - & $32 \pm 2$ & - & - & $35 \pm 5$ & $1 \pm 1$ & $\mathbf{T r}$. & - & - \\
\hline $2790-2800$ & $850.4-853.4$ & $I$ & $21 \pm 6$ & $1 \pm 1$ & $4 \pm 1$ & - & - & - & $29 \pm 2$ & - & - & $39 \pm 5$ & $2 \pm 1$ & $\mathbf{T r}$ & - & - \\
\hline $2840-2850$ & $865.6-868.7$ & I & $25 \pm 8$ & $\mathbf{T r}$ & - & - & $\mathbf{T r}$ & - & $42 \pm 2$ & - & - & $34 \pm 4$ & $2 \pm 1$ & - & - & - \\
\hline $2890-2900$ & $880.9-883.9$ & $I$ & $15 \pm 5$ & Ir. & - & - & - & - & $34 \pm 2$ & - & - & $47 \pm 6$ & $1 \pm 1$ & $2 \pm 1$ & - & - \\
\hline $2940-2950$ & $896.1-899.2$ & $\mathbf{I}$ & $1 \pm 1$ & $1 \pm 1$ & - & - & $2 \pm 1$ & - & $36 \pm 2$ & - & - & $55 \pm 6$ & $\mathbf{T r}$. & - & - & - \\
\hline $2980-2990$ & $908.3-911.4$ & I & $6 \pm 2$ & $1 \pm 1$ & - & - & $6 \pm 1$ & - & $29 \pm 2$ & - & - & $55 \pm 6$ & $1 \pm 1$ & - & - & - \\
\hline $3030-3040$ & $923.5-926.6$ & I & $11 \pm 3$ & Tr. & $1 \pm 1$ & - & Tr. & - & $30 \pm 2$ & - & - & $53 \pm 6$ & $1 \pm 1$ & $1 \pm 1$ & - & - \\
\hline $3080-3090$ & $938.8-941.8$ & $\mathbf{I}$ & $15 \pm 5$ & Tr. & - & - & - & - & $44 \pm 2$ & - & - & $34 \pm 4$ & $\mathbf{T r}$ & Tr. & - & $3 \pm 1$ \\
\hline $3130-3140$ & $954.0-957.1$ & $I$ & $6 \pm 2$ & $\mathbf{T r}$ & $2 \pm 1$ & - & $5 \pm 1$ & - & $34 \pm 2$ & - & - & $50 \pm 6$ & - & $1 \pm 1$ & - & $\mathbf{T r} .^{\bullet}, \mathrm{Tr}$ \\
\hline $3160-3170$ & $963.2-966.2$ & I & $13 \pm 4$ & $\mathbf{T r}$ & $3 \pm 1$ & - & $11 \pm 1$ & - & $37 \pm 2$ & - & - & $39 \pm 5$ & - & - & - & -9 \\
\hline $3230-3240$ & $984.5-987.6$ & I & $5 \pm 2$ & - & $\mathbf{T r}$ & - & $19 \pm 1$ & - & $38 \pm 2$ & - & - & $37 \pm 4$ & - & - & - & $\operatorname{Tr} .9$ \\
\hline $3270-3280$ & $996.7-999.7$ & I & $5 \pm 2$ & - & $2 \pm 1$ & - & $13 \pm 1$ & - & $36 \pm 2$ & - & - & $43 \pm 5$ & - & - & - & - \\
\hline $3320-3330$ & $1011.9-1015.0$ & $\mathbf{E}$ & $25 \pm 10$ & - & $4 \pm 1$ & - & - & - & $38 \pm 5$ & - & - & $25 \pm 5$ & $\mathbf{T r}$. & $1 \pm 1$ & - & $7 \pm 3^{9}$ \\
\hline $3370-3380$ & $1027.2-1030.2$ & I & $22 \pm 7$ & - & $2 \pm 1$ & - & - & - & $35 \pm 2$ & - & - & $35 \pm 4$ & $1 \pm 1$ & $\mathbf{T r}$. & - & $4 \pm 19$ \\
\hline $3410-3420$ & $1039.4-1042.4$ & I & $7 \pm 2$ & - & $3 \pm 1$ & - & $6 \pm 1$ & - & $35 \pm 2$ & - & - & $14 \pm 5$ & $\mathbf{T r}$ & - & - & $\operatorname{sr} \cdot \bullet, 1 \pm 19$ \\
\hline $3453 C$ & 1052.5 & $\mathbf{I}$ & $6 \pm 2$ & - & $2 \pm 1$ & - & $13 \pm 1$ & - & $38 \pm 2$ & - & - & $39 \pm 5$ & - & $\mathbf{T r}$ & - & - \\
\hline $3480-3490$ & $1060.7-1063.8$ & I & $7 \pm 2$ & - & $7 \pm 1$ & - & $4 \pm 1$ & - & $40 \pm 2$ & - & - & $42 \pm 5$ & - & $1 \pm 1$ & - & -.9 \\
\hline $3510-3520$ & $1069 . \approx-1072.9$ & I & $11 \pm 3$ & - & - & - & - & - & $37 \pm 2$ & - & - & $49 \pm 6$ & - & $1 \pm 1$ & - & $3 \pm 1^{9}$ \\
\hline $3550-3560$ & 1082.0 .1085 .1 & $I$ & $4 \pm 1$ & $2 \pm 1$ & - & - & - & - & $43 \pm 2$ & - & - & $45 \pm 5$ & $1 \pm 1$ & $1 \pm 1$ & - & - \\
\hline $3560-3570$ & $1085.1-1088.1$ & $\mathbf{E}$ & $7 \pm 2$ & $1 \pm 1$ & - & - & - & - & $42 \pm 5$ & - & - & $49 \pm 9$ & Ir. & $1 \pm 1$ & - & - \\
\hline $3590-3600$ & $1094.2-1097.3$ & I & $9 \pm 3$ & - & - & - & - & - & $42 \pm 2$ & - & - & $48 \pm 6$ & $\mathbf{T r}$. & - & - & - \\
\hline $3630-3640$ & $1106.4-1109.5$ & $\mathbf{I}$ & $19 \pm 6$ & - & - & - & - & - & $32 \pm 2$ & - & - & $41 \pm 5$ & $2 \pm 1$ & $1 \pm 1$ & - & $6 \pm 2^{9}, \quad 2 \pm 1_{h}^{h}$ \\
\hline $3650-3660$ & $1112.5-1115.6$ & $I$ & $19 \pm 6$ & - & - & - & - & - & $29 \pm 2$ & - & - & $42 \pm 5$ & $2 \pm 1$ & $2 \pm 1$ & - & $4 \pm 1^{9}, 1 \pm 1^{n}$ \\
\hline $3660-3670$ & $1115.6-1118.6$ & $I$ & $16 \pm 5$ & $1 \pm 1$ & - & - & - & - & $30 \pm 2$ & - & - & $51 \pm 6$ & $2 \pm 1$ & $5 \pm 1$ & - & $1 \pm 1^{n}, \operatorname{Tr} .^{2}$ \\
\hline $3690-3700$ & $1124.7-1127.8$ & $I$ & $15 \pm 5$ & - & - & - & $3 \pm 1$ & - & $36 \pm 2$ & - & - & $38 \pm 4$ & $1 \pm 1$ & $1 \pm 1$ & - & $4 \pm 1^{g}, 1 \pm 1_{h}^{h}$ \\
\hline $3720-3730$ & $1133.9-1136.9$ & $=$ & $20 \pm 8$ & - & - & - & $2 \pm 1$ & - & $27 \pm 4$ & - & - & $41 \pm 8$ & $1 \pm 1$ & $1 \pm 1$ & - & $3 \pm 19,1 \pm 1^{h}$ \\
\hline $3750-3760$ & $1143.0-1146.0$ & $\mathbf{I}$ & $15 \pm 5$ & - & $\mathbf{T r}$ & - & - & - & $33 \pm 2$ & - & - & $36 \pm 4$ & $1 \pm 2$ & $1 \pm 1$ & $8 \pm 1$ & $\operatorname{tr} \cdot \because t^{t^{9}}$ \\
\hline $3790-3800$ & $1155.2-1158.2$ & $\mathbf{I}$ & $14 \pm 4$ & - & $\cdots$ & - & - & - & $35 \pm 2$ & - & - & $33 \pm 3$ & - & $4 \pm 1$ & $21 \pm 2$ & $1 \pm 1^{2}$ \\
\hline $3820-3830$ & $1154.3-1167.4$ & $\mathbf{I}$ & $9 \pm 3$ & - & $\mathbf{T r}$ & - & - & - & $44 \pm 2$ & - & - & $19 \pm 2$ & - & $2 \pm 1$ & $22 \pm 2$ & - \\
\hline
\end{tabular}




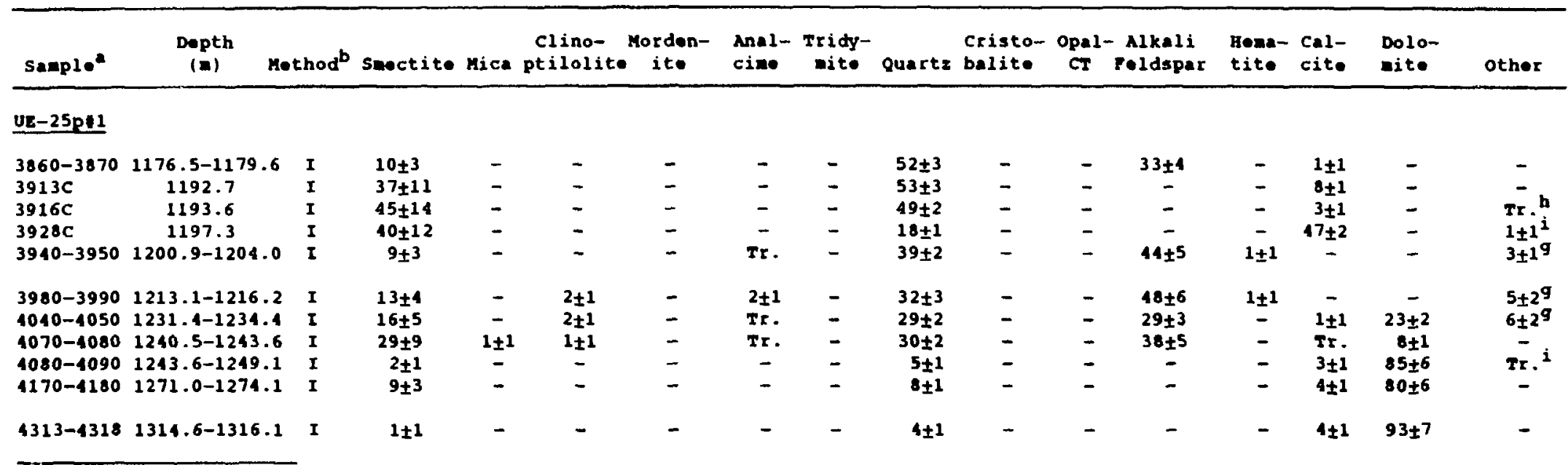

Esamples followed by SW are sidewall samples; those followed by $C$ are drill core sanples. The reanining samples are all drill cuttings. $D_{X}=$ Run using the Internal standard Mothod; $z=$ kun using the external standard Mothod.

cGlass.

dxaolinite.

inumontite.

tgornblende.

gillite.

hehlorite.

irluorite. 


\begin{tabular}{|c|c|c|c|c|c|c|c|c|c|c|c|c|c|c|c|c|}
\hline Samplea & $\begin{array}{l}\text { Depth } \\
\text { (m) M }\end{array}$ & Method & smoctite & Mica & $\begin{array}{c}\text { Clino- } \\
\text { ptilolite }\end{array}$ & $\begin{array}{l}\text { Morden- } \\
\text { ite }\end{array}$ & $\begin{array}{l}\text { Anal- } \\
\text { cime }\end{array}$ & $\begin{array}{c}\text { Tridy- } \\
\text { nite }\end{array}$ & Quartz & $\begin{array}{l}\text { Cristo- } \\
\text { balice }\end{array}$ & $\begin{array}{c}\text { Ope 1- } \\
\text { CT }\end{array}$ & $\begin{array}{c}\text { Alkali } \\
\text { reldspar }\end{array}$ & calcite & Glass & Howatite & other \\
\hline \multicolumn{17}{|l|}{ USW G-1 } \\
\hline 292 & 89.0 & I & - & $1 \pm 1$ & - & - & - & $4 \pm 1$ & $1 \pm 1$ & $15 \pm 1$ & - & $72 \pm 10$ & - & - & Tr. & - \\
\hline 352 & 107.3 & $I$ & - & $\mathbf{T r}$. & $3 \pm 1$ & - & - & $9 \pm 1$ & - & $8 \pm 4$ & - & $73 \pm 12$ & - & - & $1 \pm 1$ & - \\
\hline 399 & 121.6 & $I$ & - & $\mathbf{T r}$ & $=$ & - & - & $12 \pm 1$ & - & $5 \pm 2$ & - & $78 \pm 13$ & - & - & $1 \pm 1$ & $\operatorname{Tr} .^{C}$ \\
\hline 450 & 137.2 & $\mathbf{I}$ & $3 \pm 1$ & $\mathbf{T r}$ & - & - & - & $24 \pm 3$ & $2 \pm 1$ & $6 \pm 3$ & - & $67 \pm 15$ & - & - & $1 \pm 1$ & - \\
\hline 553 & 168.6 & $I$ & $2 \pm 1$ & - & - & - & - & $7 \pm 1$ & $4 \pm 1$ & $27 \pm 2$ & - & $61 \pm 9$ & - & - & $1 \pm 1$ & - \\
\hline 619 & 188.7 & I & $2 \pm 1$ & Tr. & - & - & - & $20 \pm 2$ & $2 \pm 1$ & $11 \pm 1$ & - & $65 \pm 14$ & Tr. & - & $1 \pm 1$ & - \\
\hline 673 & 205.1 & I & $1 \pm 1$ & $\mathbf{T r}$ & - & - & - & $6 \pm 1$ & $4 \pm 1$ & $24 \pm 1$ & - & $64 \pm 10$ & - & - & $1 \pm 1$ & - \\
\hline 722 & 220.1 & $\mathbf{I}$ & $2 \pm 1$ & $\mathbf{T r}$ & - & - & $\rightarrow$ & TI. & $3 \pm 1$ & $22 \pm 1$ & - & $72 \pm 9$ & - & - & $=$ & - \\
\hline 757 & 230.7 & $\mathbf{I}$ & $1 \pm 1$ & $\mathbf{T r}$ & - & - & - & $6 \pm 1$ & $4 \pm 1$ & $27 \pm 1$ & - & $67 \pm 9$ & - & - & $1 \pm 1$ & - \\
\hline 819 & 249.6 & I & $2 \pm 1$ & $\mathbf{T r}$ & - & - & - & $4 \pm 1$ & $4 \pm 1$ & $26 \pm 1$ & - & $65 \pm 9$ & - & - & $\mathrm{Tr}$ & - \\
\hline 874 & 266.4 & I & $2 \pm 1$ & - & - & - & - & $7 \pm 1$ & $19 \pm 1$ & $5 \pm 2$ & - & $66 \pm 10$ & - & - & Tr. & - \\
\hline 936 & 285.3 & $I$ & - & - & - & - & - & $=$ & $28 \pm 1$ & $=$ & - & $40 \pm 5$ & $35 \pm 1$ & - & - & Tr. \\
\hline 995 & 303.3 & I & $3 \pm 1$ & - & - & - & - & $5 \pm 1$ & $4 \pm 1$ & $23 \pm 1$ & - & $64 \pm 9$ & $=$ & - & Tr. & - \\
\hline 1063 & 324.0 & $I$ & $2 \pm 1$ & Tr. & - & - & - & $7 \pm 1$ & $11 \pm 1$ & $16 \pm 1$ & - & $64 \pm 10$ & - & - & $1 \pm 2$ & - \\
\hline 1104 & 336.5 & $I$ & $2 \pm 1$ & - & - & - & - & $4 \pm 1$ & $11 \pm 1$ & $20 \pm 1$ & - & $59 \pm 8$ & - & - & $1 \pm 1$ & - \\
\hline 1123 & 342.3 & I & $2 \pm 1$ & $\mathbf{T r}$ & - & - & - & $6 \pm 1$ & $7 \pm 1$ & $18 \pm 1$ & - & $64 \pm 10$ & - & - & Tr. & - \\
\hline 1179 & 359.4 & I & $3 \pm 1$ & - & - & - & - & $3 \pm 1$ & $15 \pm 1$ & $19 \pm 1$ & - & $56 \pm 7$ & - & - & $1 \pm 1$ & - \\
\hline 1191 & 363.0 & $\mathbf{I}$ & $4 \pm 1$ & - & - & - & - & $6 \pm 1$ & $11 \pm 1$ & $16 \pm 1$ & - & $63 \pm 9$ & - & - & $1 \pm 1$ & - \\
\hline 1240 & 378.0 & $I$ & $2 \pm 1$ & Tr. & - & - & - & $2 \pm 1$ & $15 \pm 1$ & $17 \pm 1$ & - & $64 \pm 8$ & - & - & $\mathbf{T r}$ & - \\
\hline 1274 & 388.3 & I & $2 \pm 1$ & $\mathbf{T r}$ & $\mathbf{T r}$ & - & - & $2 \pm 1$ & $16 \pm 1$ & $18 \pm 1$ & - & $62 \pm^{8}$ & - & - & Tr. & - \\
\hline 1281 & 390.4 & I & $2 \pm 1$ & $\mathbf{T r}$ & - & - & - & $2 \pm 1$ & $18 \pm 1$ & $14 \pm 1$ & - & $66 \pm 8$ & - & - & Tr. & - \\
\hline 1286 & 392.0 & I & $33 \pm 7$ & - & $10 \pm 1$ & - & - & - & $3 \pm 1$ & $16 \pm 1$ & - & $38 \pm 5$ & - & - & - & - \\
\hline 1319 & 402.0 & I & $=$ & - & - & - & - & - & Tr. & - & $3 \pm 1$ & $5 \pm 1$ & - & $92 \pm 2$ & - & - \\
\hline 1341 & 408.7 & I & - & - & $2 \pm 1$ & - & - & - & Tr. & - & $4 \pm 1$ & $6 \pm 1$ & - & $87 \pm 2$ & - & - \\
\hline 1357 & 413.6 & $I$ & $1 \pm 1$ & - & - & - & - & - & Tr. & - & $3 \pm 1$ & $7 \pm 1$ & - & $88 \pm 2$ & - & - \\
\hline 1392 & 424.3 & I & $\mathrm{Tr}$ & - & $6 \pm 1$ & - & - & - & Tr. & - & $2 \pm 1$ & $3 \pm 1$ & - & $14 \pm 2$ & - & - \\
\hline 1400 & 426.7 & I & Tr. & - & $83 \pm 10$ & - & - & - & $2 \pm 1$ & - & $12 \pm 2$ & $2 \pm 1$ & - & $=$ & - & - \\
\hline 1492 & 454.8 & I & Tr. & - & $63 \pm 17$ & $11 \pm 3$ & - & - & $3 \pm 1$ & - & $19 \pm 4$ & $5 \pm 1$ & - & - & - & - \\
\hline 1539 & 469.1 & I & $1 \pm 1$ & $\mathrm{Tr}$ & $46 \pm 7$ & Tr. & - & - & $11 \pm 1$ & - & $19 \pm 4$ & $17 \pm 3$ & - & - & - & - \\
\hline 1561 & 475.8 & I & Tr. & - & $62 \pm 18$ & $17 \pm 4$ & - & - & $2 \pm 1$ & - & $11 \pm 2$ & $6 \pm^{2}$ & - & - & - & - \\
\hline 1639 & 499.6 & I & Tr. & - & $60 \pm 14$ & $12 \pm 3$ & - & - & $3 \pm 1$ & - & $12 \pm 2$ & $10 \pm 2$ & - & - & - & - \\
\hline 1693 & 516.0 & $t$ & - & - & $50 \pm 15$ & $16 \pm 4$ & - & - & $3 \pm 1$ & - & $15 \pm 3$ & $13 \pm 3$ & - & - & - & - \\
\hline 1748 & 532.8 & $I$ & - & - & $55 \pm 17$ & $11 \pm 2$ & - & - & $3 \pm 1$ & - & $26 \pm 5$ & $4 \pm 1$ & - & - & - & - \\
\hline 1774 & 540.7 & I & Tr & $1 \pm 1$ & $19 \pm 8$ & $11 \pm 2$ & - & - & $13 \pm 1$ & $4 \pm 1$ & $=$ & $19 \pm 4$ & - & - & - & - \\
\hline 1784 & 543.8 & I & $4 \pm 1$ & $T \vec{r}$. & $25 \pm 5$ & $13 \pm 4$ & - & $\mathbf{T r}$ & $9 \pm 1$ & $7 \pm 2$ & - & $41 \pm 9$ & $1 \pm 1$ & - & $=$ & - \\
\hline
\end{tabular}




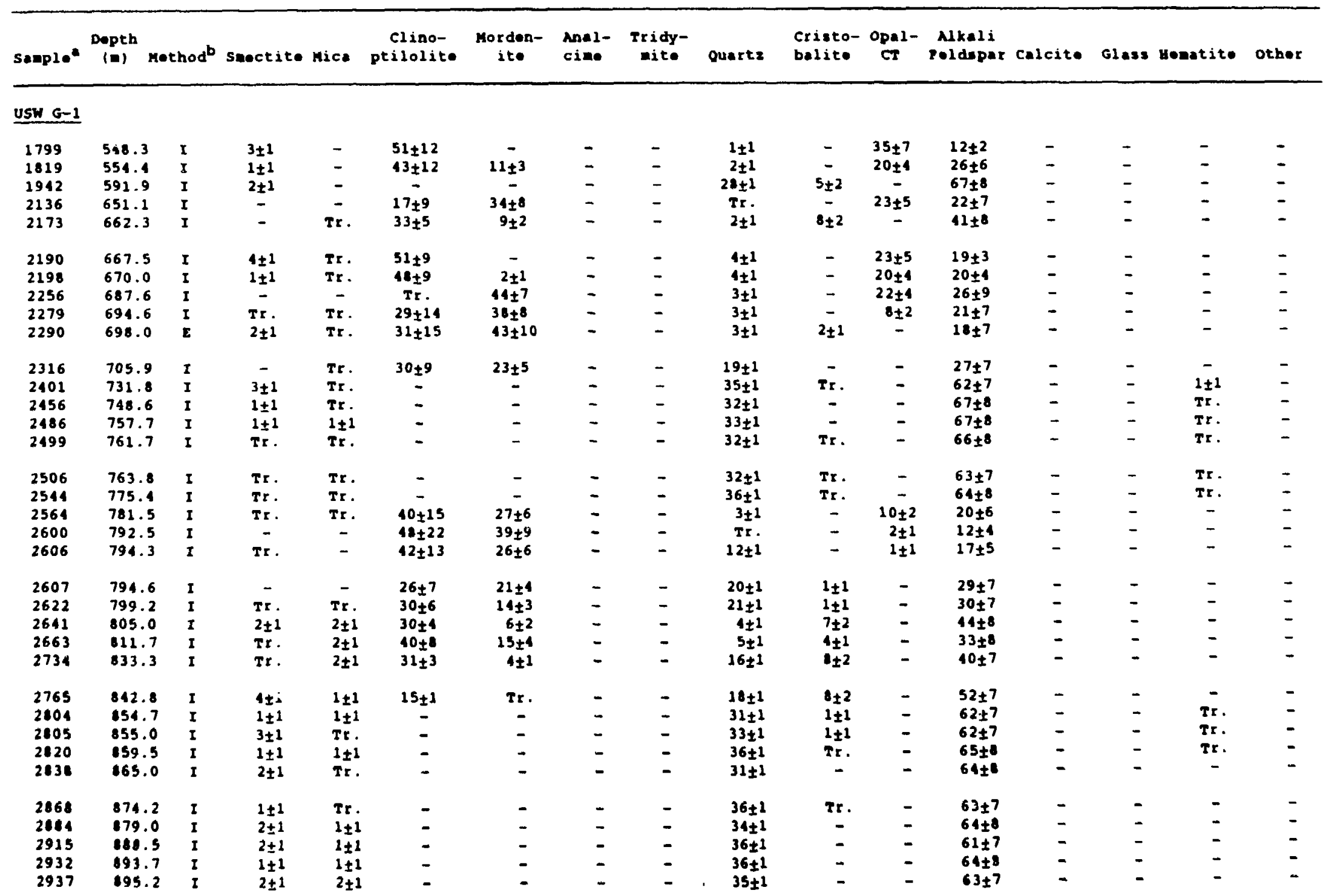




\begin{tabular}{|c|c|c|c|c|c|c|c|c|c|c|c|c|c|c|c|c|}
\hline Samplo" & $\begin{array}{l}\text { Dopth } \\
\text { (a) }\end{array}$ & Method ${ }^{b}$ : & smectite & Mica & $\begin{array}{l}\text { Clino- } \\
\text { ptilolite }\end{array}$ & $\begin{array}{l}\text { Morden- } \\
\text { ite }\end{array}$ & $\begin{array}{l}\text { Anal- } \\
\text { cine }\end{array}$ & $\begin{array}{l}\text { Tridy- } \\
\text { nite }\end{array}$ & Quartz & $\begin{array}{l}\text { Cristo- } \\
\text { balite }\end{array}$ & $\begin{array}{c}\text { Ope1- } \\
\text { CT }\end{array}$ & $\begin{array}{l}\text { Alkali } \\
\text { reldspar }\end{array}$ & Calcite & Glass & Henatite & Other \\
\hline \multicolumn{17}{|l|}{ USW G-1 } \\
\hline 2948 & 898.6 & $\mathbf{I}$ & $2 \pm 1$ & $1 \pm 1$ & $2 \pm 1$ & - & - & - & $30 \pm 1$ & - & - & $67 \pm 8$ & $1 \pm 1$ & - & - & - \\
\hline 2966 & 904.0 & $I$ & $1 \pm 1$ & $2 \pm 1$ & - & - & - & - & $36 \pm 1$ & - & - & $61 \pm 7$ & - & - & - & - \\
\hline 2981 & 908.6 & I & $3 \pm 1$ & $1 \pm 1$ & - & - & - & - & $37 \pm 1$ & - & - & $58 \pm 7$ & - & - & - & - \\
\hline 3018 & 919.9 & I & $18 \pm 4$ & $1 \pm 1$ & $7 \pm 1$ & - & $4 \pm 1$ & - & $33 \pm 1$ & - & - & $34 \pm 4$ & $\mathbf{T r}$ & - & - & - \\
\hline 3079 & 938.5 & I & $11 \pm 2$ & $1 \pm 1$ & $13 \pm 1$ & - & $5 \pm 1$ & - & $36 \pm 1$ & - & $\cdots$ & $34 \pm 5$ & - & - & - & - \\
\hline 3167 & 965.3 & I & $10 \pm 2$ & $1 \pm 1$ & $19 \pm 1$ & - & $5 \pm 1$ & - & $31 \pm 1$ & - & - & $31 \pm 4$ & - & - & - & - \\
\hline 3288 & 1002.2 & I & $17 \pm 3$ & Tr. & $40 \pm 2$ & - & $1 \pm 1$ & - & $18 \pm 1$ & - & - & $20 \pm 3$ & - & - & - & - \\
\hline 3401 & 1036.6 & $\mathbf{I}$ & $4 \pm 1$ & Tr. & $10 \pm 1$ & - & $2 \pm 1$ & - & $38 \pm 1$ & - & - & $43 \pm 6$ & Tr. & - & - & - \\
\hline 3523 & 1073.8 & I & $7 \pm 1$ & $\mathrm{Tr}$. & $13 \pm 1$ & - & $1 \pm 1$ & - & $32 \pm 1$ & - & - & $46 \pm 6$ & $2 \pm 1$ & - & - & $2 \pm 1^{d}$ \\
\hline 3621 & 1103.7 & I & $24 \pm 5$ & - & - & - & - & - & $3 \pm 1$ & $6 \pm 2$ & - & $59 \pm 7$ & $2 \pm 1$ & - & $2 \pm 1$ & $=$ \\
\hline 3810 & 1161.3 & I & $65 \pm 13$ & - & - & - & - & - & - & - & - & $37 \pm 4$ & $2 \pm 1$ & - & - & - \\
\hline 3940 & 1200.9 & I & $48 \pm 10$ & $\mathrm{Tr}$ & $2 \pm 1$ & - & $2 \pm 1$ & - & $19 \pm 1$ & - & - & $23 \pm 3$ & Tr. & - & - & $2 \pm 1 d$ \\
\hline 4246 & 1294.2 & I & $17 \pm 3$ & $\mathrm{Tr}$. & - & - & $6 \pm 1$ & - & $38 \pm 1$ & - & - & $38 \pm 5$ & - & - & - & $=$ \\
\hline 4400 & 1341.1 & $I$ & $7 \pm 1$ & $1 \pm 1$ & - & - & $13 \pm 1$ & - & $39 \pm 1$ & - & - & $12 \pm 5$ & Tr. & - & - & - \\
\hline 4503 & 1372.5 & I & $4 \pm 1$ & $1 \pm 1$ & - & - & $19 \pm 1$ & - & $39 \pm 1$ & - & - & $37 \pm 4$ & $2 \pm 1$ & - & - & - \\
\hline 4555 & 1388.4 & I & $21 \pm 4$ & $\mathbf{T r}$ & - & - & $2 \pm 1$ & - & $22 \pm 1$ & - & - & $47 \pm 6$ & $\mathrm{Tr}$ & - & Tr. & - \\
\hline 4612 & 1405.7 & I & $13 \pm 3$ & $2 \pm 1$ & - & - & $7 \pm 1$ & - & $34 \pm 1$ & - & - & $41 \pm 5$ & - & - & Tr. & - \\
\hline 4626 & 1410.0 & I & $4 \pm 1$ & - & - & - & - & - & $42 \pm 1$ & - & - & $52 \pm 6$ & $4 \pm 1$ & - & - & - \\
\hline 4652 & 1417.9 & I & $7 \pm 1$ & Tr. & - & - & $11 \pm 1$ & - & $40 \pm 1$ & - & - & $38 \pm 5$ & $=$ & - & - & - \\
\hline 4700 & 1432.6 & I & $6 \pm 1$ & Tr. & - & - & $7 \pm 1$ & - & $46 \pm 1$ & - & - & $40 \pm 5$ & - & - & - & - \\
\hline 4750 & 1447.8 & I & $8 \pm 2$ & $1 \pm 1$ & - & - & $10 \pm 1$ & - & $37 \pm 1$ & - & - & $14 \pm 5$ & - & - & - & - \\
\hline 4805 & 1464.6 & I & $8 \pm 2$ & $\mathrm{Tr}$. & - & - & $9 \pm 1$ & - & $38 \pm 1$ & - & - & $43 \pm 5$ & $2 \pm 1$ & - & - & - \\
\hline 4848 & 1477.7 & I & $6 \pm 1$ & $T x$. & - & - & $11 \pm 1$ & - & $11 \pm 1$ & - & - & $38 \pm 5$ & $=$ & - & - & - \\
\hline 4876 & 1486.2 & $I$ & $4 \pm 1$ & Tr. & - & - & $4 \pm 1$ & - & $45 \pm 1$ & - & - & $45 \pm 5$ & $2 \pm 1$ & - & - & - \\
\hline 4912 & 1497.2 & I & $7 \pm 1$ & $\mathbf{T r}$ & - & - & $8 \pm 1$ & - & $43 \pm 1$ & - & - & $37 \pm 4$ & - & - & - & - \\
\hline 4941 & 1506.0 & I & $27 \pm 5$ & Tr. & - & - & $7 \pm 1$ & - & $27 \pm 1$ & - & - & $25 \pm 3$ & $6 \pm 1$ & - & - & - \\
\hline 4958 & 1511.2 & $\mathbf{I}$ & $8 \pm 2$ & - & - & - & $17 \pm 1$ & - & $46 \pm 1$ & - & - & $30 \pm 4$ & $=$ & - & - & - \\
\hline 1998 & 1523.4 & I & $30 \pm 6$ & $\mathbf{T r}$ & - & - & $3 \pm 1$ & - & $39 \pm 1$ & - & - & $27 \pm 3$ & Tr. & - & - & $\operatorname{Ir.}{ }^{\mathrm{C}}$ \\
\hline 5026 & 1531.9 & I & $5 \pm 1$ & $\mathbf{T r}$ & - & - & $2 \pm 1$ & - & $45 \pm 1$ & - & - & $51 \pm 6$ & - & - & - & - \\
\hline 5049 & 1538.9 & I & $52 \pm 10$ & $2 \pm 1$ & - & - & - & - & $5 \pm 1$ & - & - & $28 \pm 3$ & $\mathbf{T r}$ & - & $\mathbf{T r}$ & - \\
\hline 5093 & 1552.3 & $I$ & $5 \pm 1$ & Tr. & $3 \pm 1$ & - & $2 \pm 1$ & - & $39 \pm 1$ & - & - & $47 \pm 6$ & $2 \pm 1$ & - & $\mathbf{T r}$ & $\operatorname{sr} \cdot \frac{d}{d}$ \\
\hline 5126 & 1562.4 & 1 & $2 \pm 1$ & Te. & - & - & $15 \pm 1$ & - & $50 \pm 1$ & - & - & $33 \pm 4$ & $\mathbf{T r}$. & - & - & Tr. \\
\hline 5167 & 1574.9 & $\mathbf{I}$ & $2 \pm 1$ & Tr. & - & - & $14 \pm 1$ & - & $16 \pm 1$ & - & - & $40 \pm 5$ & Tr. & - & - & Ix. \\
\hline 5212 & 1580.6 & I & $1 \pm 1$ & Tr. & - & - & $16 \pm 1$ & - & $48 \pm 1$ & - & - & $38 \pm 5$ & - & - & - & Tr. \\
\hline 5253 & $1601 \cdot 1$ & I & $1 \pm 1$ & Tr. & - & - & $27 \pm 1$ & - & $36 \pm 1$ & - & - & $35 \pm 4$ & Tr. & - & - & - \\
\hline
\end{tabular}




\begin{tabular}{|c|c|c|c|c|c|c|c|c|c|c|c|c|c|c|c|c|}
\hline Sanplea & $\begin{array}{l}\text { Depth } \\
\text { (a) }\end{array}$ & Method ${ }^{b}$ & smectite & Mica & $\begin{array}{l}\text { Clino- } \\
\text { ptilolite }\end{array}$ & $\begin{array}{l}\text { Morden- } \\
\text { ite }\end{array}$ & $\begin{array}{l}\text { Anal- } \\
\text { cinos }\end{array}$ & $\begin{array}{l}\text { Iridy- } \\
\text { mite }\end{array}$ & Quartz & $\begin{array}{l}\text { Cristo- } \\
\text { balite }\end{array}$ & $\begin{array}{c}\text { Opal- } \\
\text { CT }\end{array}$ & $\begin{array}{l}\text { Alkali } \\
\text { roldepar }\end{array}$ & Calcite & Glass & Henatite & other \\
\hline \multicolumn{17}{|l|}{ USW $G-1$} \\
\hline $\begin{array}{l}5296 \\
5310 \\
5311 \\
5329 \\
5338\end{array}$ & $\begin{array}{l}1614.2 \\
1618.5 \\
1618.8 \\
1624.3 \\
1627.0\end{array}$ & $\begin{array}{l}I \\
I \\
I \\
I \\
I\end{array}$ & $\begin{array}{r}2 \pm 1 \\
29 \pm 6 \\
6 \pm 1 \\
11 \pm 2 \\
11 \pm 8\end{array}$ & $\begin{array}{c}- \\
\overline{T r} . \\
- \\
\overline{T r} .\end{array}$ & $\begin{array}{c}- \\
- \\
1 \pm 1 \\
- \\
1 \pm 1\end{array}$ & $\begin{array}{l}- \\
- \\
- \\
-\end{array}$ & $\begin{array}{c}19 \pm 1 \\
- \\
12 \pm 1 \\
-\end{array}$ & $\begin{array}{l}- \\
- \\
- \\
-\end{array}$ & $\begin{array}{l}37 \pm 1 \\
23 \pm 1 \\
46 \pm 1 \\
40 \pm 1 \\
28 \pm 1\end{array}$ & $\begin{array}{l}- \\
- \\
- \\
-\end{array}$ & $\begin{array}{l}- \\
- \\
- \\
-\end{array}$ & $\begin{array}{l}39 \pm 5 \\
52 \pm 6 \\
45 \pm 5 \\
40 \pm 5 \\
26 \pm 3\end{array}$ & $\begin{array}{c}- \\
- \\
1 \pm 1 \\
- \\
\mathrm{Tr}\end{array}$ & $\begin{array}{l}- \\
- \\
-\end{array}$ & $\begin{array}{l}- \\
\overline{-} \\
-\end{array}$ & $\begin{array}{l}\text { Tr.d } \\
- \\
=d \\
=\end{array}$ \\
\hline $\begin{array}{l}5348 \\
5378 \\
5412 \\
5433 \\
5458\end{array}$ & $\begin{array}{l}1630.1 \\
1639.2 \\
1649.6 \\
1656.0 \\
1663.6\end{array}$ & $\begin{array}{l}I \\
I \\
I \\
I \\
I\end{array}$ & $\begin{array}{r}9 \pm 2 \\
16 \pm 9 \\
5 \pm 1 \\
35 \pm 7 \\
26 \pm 5\end{array}$ & $\begin{array}{l}T r \\
T r \\
1 \pm 1 \\
2 \pm 1 \\
T r\end{array}$ & $\begin{array}{c}T r \\
15 \pm 1 \\
- \\
20 \pm 1 \\
25 \pm 2\end{array}$ & $\begin{array}{l}- \\
- \\
- \\
-\end{array}$ & $\begin{array}{c}- \\
- \\
12 \pm 1 \\
-\end{array}$ & $\begin{array}{l}- \\
\overline{-} \\
\overline{-}\end{array}$ & $\begin{array}{r}35 \pm 1 \\
17 \pm 1 \\
40 \pm 1 \\
9 \pm 1 \\
18 \pm 1\end{array}$ & $\begin{array}{l}- \\
- \\
- \\
-\end{array}$ & $\begin{array}{l}- \\
- \\
-\end{array}$ & $\begin{array}{l}14 \pm 5 \\
24 \pm 3 \\
10 \pm 5 \\
27 \pm 4 \\
28 \pm 4\end{array}$ & $\begin{array}{c}9 \pm 1 \\
- \\
- \\
2 \pm 1 \\
\mathrm{Tr} .\end{array}$ & $\begin{array}{l}- \\
z \\
-\end{array}$ & $\begin{array}{l}- \\
\overline{-} \\
-\end{array}$ & $\begin{array}{l}- \\
\operatorname{Tr} \cdot d \\
-\end{array}$ \\
\hline $\begin{array}{l}5477 \\
5498 \\
5534 \\
5560 \\
5596\end{array}$ & $\begin{array}{l}1669.4 \\
1675.1 \\
1686.1 \\
1694.7 \\
1705.7\end{array}$ & $\begin{array}{l}I \\
I \\
I \\
I \\
I\end{array}$ & $\begin{array}{r}5 \pm 1 \\
5 \pm 1 \\
30 \pm 8 \\
5 \pm 1 \\
2 \pm 1\end{array}$ & $\begin{array}{l}- \\
2 r \\
2 \pm 1 \\
1 \pm 1 \\
2 \pm 1\end{array}$ & $\begin{array}{r}- \\
- \\
20 \pm 1 \\
16 \pm 1 \\
1 \pm 1\end{array}$ & $\begin{array}{c}- \\
- \\
\overline{23} \pm 7 \\
-\end{array}$ & $\begin{array}{c}12 \pm 1 \\
17 \pm 1 \\
4 \pm 1 \\
- \\
7 \pm 1\end{array}$ & $\begin{array}{l}- \\
= \\
-\end{array}$ & $\begin{array}{r}14 \pm 1 \\
38 \pm 1 \\
2 \pm 1 \\
21 \pm 1 \\
34 \pm 1\end{array}$ & $\begin{array}{l}- \\
\overline{-} \\
- \\
-\end{array}$ & $\begin{array}{l}- \\
- \\
- \\
-\end{array}$ & $\begin{array}{l}36 \pm 4 \\
41 \pm 5 \\
24 \pm 3 \\
33 \pm 8 \\
50 \pm 6\end{array}$ & $\begin{array}{l}- \\
\operatorname{Tr} \\
1 \pm 1 \\
\operatorname{Tr} \\
2 \pm 1\end{array}$ & $\begin{array}{l}- \\
- \\
-\end{array}$ & $\begin{array}{l}- \\
= \\
=\end{array}$ & $\begin{array}{l}\overline{-} \\
\overline{2 \pm 1} c \\
\operatorname{Tr} \cdot d \\
\operatorname{Tr} . d\end{array}$ \\
\hline $\begin{array}{l}5637 \\
5679 \\
5699 \\
5746 \\
5803\end{array}$ & $\begin{array}{l}1718.2 \\
1731.0 \\
1737.1 \\
1751.4 \\
1768.8\end{array}$ & $\begin{array}{l}I \\
I \\
I \\
I \\
I\end{array}$ & $\begin{array}{r}3 \pm 1 \\
20 \pm 4 \\
5 \pm 1 \\
12 \pm 2 \\
10 \pm 2\end{array}$ & $\begin{array}{l}1 \pm 1 \\
\mathrm{Tr} . \\
\mathrm{Tr} . \\
\mathrm{Tr} . \\
\mathrm{Tr} .\end{array}$ & $\begin{array}{l}- \\
- \\
-\end{array}$ & $\begin{array}{l}- \\
- \\
- \\
-\end{array}$ & $\begin{array}{r}16 \pm 1 \\
13 \pm 1 \\
9 \pm 1 \\
10 \pm 1 \\
=\end{array}$ & $\begin{array}{l}- \\
\overline{-} \\
-\end{array}$ & $\begin{array}{l}38 \pm 1 \\
24 \pm 1 \\
33 \pm 1 \\
32 \pm 1 \\
29 \pm 1\end{array}$ & $\begin{array}{l}- \\
- \\
-\end{array}$ & $\begin{array}{l}- \\
- \\
- \\
-\end{array}$ & $\begin{array}{l}12 \pm 5 \\
36 \pm 4 \\
50 \pm 6 \\
16 \pm 5 \\
54 \pm 6\end{array}$ & $\begin{array}{l}\text { Tr. } \\
\text { Tr. } \\
T r . \\
T r . \\
2 \pm 1\end{array}$ & $\begin{array}{l}- \\
- \\
- \\
-\end{array}$ & $\begin{array}{l}= \\
= \\
=\end{array}$ & $\begin{array}{l}1 \pm 1 d \\
\operatorname{Tr} d \\
\operatorname{Tr} d \\
1 \pm 1 d \\
1 \pm 1\end{array}$ \\
\hline $\begin{array}{l}5847 \\
5898 \\
5947 \\
5980\end{array}$ & $\begin{array}{l}1782.2 \\
1797.7 \\
1812.6 \\
1822.7\end{array}$ & $\begin{array}{l}I \\
I \\
I \\
I\end{array}$ & $\begin{array}{r}13 \pm 3 \\
7 \pm 1 \\
12 \pm 2 \\
14 \pm 3\end{array}$ & $\begin{array}{l}\text { Tr. } \\
\text { Tr. } \\
\text { Tr. }\end{array}$ & $\begin{array}{l}- \\
2 \pm 1 \\
1 \pm 1\end{array}$ & $\begin{array}{l}- \\
- \\
-\end{array}$ & $\begin{array}{c}- \\
12 \pm 1 \\
25 \pm 1 \\
-\end{array}$ & $\begin{array}{l}- \\
= \\
-\end{array}$ & $\begin{array}{l}29 \pm 1 \\
42 \pm 1 \\
22 \pm 1 \\
29 \pm 1\end{array}$ & $\begin{array}{l}- \\
-\end{array}$ & $\begin{array}{l}- \\
-\end{array}$ & $\begin{array}{l}34 \pm 6 \\
34 \pm 4 \\
34 \pm 4 \\
10 \pm 5\end{array}$ & $\begin{array}{l}1 \pm 1 \\
T r . \\
1 \pm 1 \\
T r .\end{array}$ & $\begin{array}{l}- \\
-\end{array}$ & $\begin{array}{l}= \\
= \\
-\end{array}$ & $\begin{array}{c}1 \pm 1^{d}, 1 \pm 1 \\
-1^{d} \\
2 \pm 1\end{array}$ \\
\hline
\end{tabular}

asaplo dosignation, e.g., 292 rofors to a saple from drill hole UsH o-l at 292-ft dopth.

$b_{1}$ - Internel standerd Mothod, $\varepsilon$ a external standerd Mothod.

Eliornblende.

¿chlorite.

thumontito(r). 
APPENDIX A (cont)

Core

\begin{tabular}{|c|c|c|c|c|c|c|c|c|c|c|c|c|c|}
\hline sanple & $\begin{array}{c}\text { Depth } \\
\text { (a) }\end{array}$ & smoctite & Mica & $\begin{array}{l}\text { clino- } \\
\text { ptilolite }\end{array}$ & $\begin{array}{l}\text { Morden- } \\
\text { ite }\end{array}$ & $\begin{array}{l}\text { Anal- } \\
\text { cine }\end{array}$ & $\begin{array}{l}\text { Tridy- } \\
\text { nite }\end{array}$ & Quartz & $\begin{array}{l}\text { Cristo- } \\
\text { balite }\end{array}$ & $\begin{array}{c}\text { Nkeli } \\
\text { Foldspar }\end{array}$ & Calcite & Glas: & other \\
\hline
\end{tabular}

\section{USW G-2}

\begin{tabular}{|c|c|c|c|c|c|c|c|c|c|c|c|c|c|}
\hline 10 & 3.0 & - & $7 \pm 3$ & - & - & - & $15 \pm 5$ & - & $40 \pm 10$ & $40 \pm 10$ & - & - & - \\
\hline 100 & 30.5 & - & $3 \pm 2$ & - & - & - & $18 \pm 7$ & - & $10 \pm 10$ & $40 \pm 10$ & - & - & - \\
\hline 200 & 61.0 & - & - & - & - & - & $10 \pm 5$ & - & $40 \pm 10$ & $10 \pm 10$ & - & - & - \\
\hline 230 & 70.1 & - & - & - & - & - & $10 \pm 5$ & - & $40 \pm 10$ & $10 \pm 10$ & - & - & - \\
\hline 270 & 82.3 & $6 \pm 4$ & - & - & - & - & - & - & $40 \pm 10$ & $10 \pm 10$ & $23 \pm 7$ & - & - \\
\hline 304 & 92.7 & - & - & - & - & - & $15 \pm 5$ & $7 \pm 3$ & $40 \pm 10$ & $40 \pm 10$ & - & - & - \\
\hline 331 & 100.9 & $40 \pm 10$ & - & - & - & - & - & - & $10 \pm 10$ & $15 \pm 5$ & - & - & - \\
\hline 338 & 103.0 & $50 \pm 10$ & - & - & - & - & - & - & - & $7 \pm 3$ & - & $10 \pm 10$ & - \\
\hline 358 & 109.1 & $23 \pm 7$ & - & - & - & - & - & $\mathbf{T r}$. & $7 \pm 3$ & $30 \pm 10$ & - & $30 \pm 10$ & - \\
\hline 395 & 120.4 & $10 \pm 5$ & $<2$ & - & - & - & - & $3 \pm 2$ & $15 \pm 5$ & $23 \pm 7$ & - & $10 \pm 10$ & - \\
\hline 501 & 152.7 & $12 \pm 8$ & $<3$ & - & - & - & - & - & $7 \pm 3$ & $15 \pm 5$ & - & $50 \pm 10$ & - \\
\hline 547 & 166.7 & $10 \pm 5$ & $3 \pm 2$ & - & - & - & - & - & $10 \pm 5$ & $15 \pm 5$ & - & $50 \pm 10$ & - \\
\hline 548 & 167.0 & - & $3 \pm 2$ & $7 \pm 3$ & - & - & - & - & $30 \pm 10$ & $10 \pm 10$ & - & - & - \\
\hline 561 & 171.0 & $15 \pm 5$ & $<\overline{3}$ & $15 \pm 5$ & - & - & - & - & $30 \pm 10$ & $32 \pm 8$ & - & - & - \\
\hline 627 & 191.1 & $10 \pm 5$ & $3 \pm 2$ & $15 \pm 5$ & - & - & - & - & $30 \pm 10$ & $10 \pm 10$ & - & - & - \\
\hline 675 & 205.7 & $40 \pm 10$ & $3 \pm 2$ & $23 \pm 7$ & - & - & - & $\mathbf{T r}$ & $7 \pm 3$ & $23 \pm 7$ & - & - & - \\
\hline 723 & 220.4 & $10 \pm 5$ & $3 \pm 2$ & - & - & - & - & - & $3 \pm 2$ & $7 \pm 3$ & $10 \pm 10$ & $23 \pm 7$ & - \\
\hline 743 & 226.5 & $35 \pm 10$ & $3 \pm 2$ & $3 \pm 2$ & - & - & - & $10 \pm 5$ & $10 \pm 5$ & $15 \pm 5$ & $10 \pm 5$ & $20 \pm 7$ & - \\
\hline 762 & 232.3 & $3 \pm 2$ & - & $75 \pm^{8}$ & - & - & $15 \pm 5$ & - & $10 \pm 5$ & $3 \pm 2$ & - & - & - \\
\hline 770 & 234.7 & $<\overline{2}$ & $7 \pm 3$ & - & - & - & - & - & $23 \pm 7$ & $70 \pm 10$ & - & - & - \\
\hline 822 & 250.5 & $7 \pm 3$ & $3 \pm 2$ & - & - & - & $15 \pm 5$ & - & $30 \pm 10$ & $40 \pm 10$ & - & - & - \\
\hline 855 & 260.6 & $3 \pm 2$ & $3 \pm 2$ & - & - & - & $10 \pm 5$ & - & $30 \pm 10$ & $40 \pm 10$ & - & - & - \\
\hline 898 & 273.7 & $<2$ & $3 \pm 2$ & $3 \pm 2$ & - & - & $7 \pm 3$ & - & $30 \pm 10$ & $50 \pm 10$ & - & - & - \\
\hline 921 & 280.7 & $15 \pm 5$ & - & - & - & - & - & $30 \pm 10$ & $23 \pm 7$ & $30 \pm 10$ & $7 \pm 3$ & - & - \\
\hline 951 & 289.9 & 12 & - & - & - & - & - & $23 \pm 7$ & $30 \pm 10$ & $30 \pm 10$ & - & - & - \\
\hline 984 & 299.9 & $6 \pm 4$ & - & - & - & - & - & $23 \pm 7$ & $15 \pm 5$ & $23 \pm 7$ & $23 \pm 7$ & - & - \\
\hline 1032 & 314.6 & $6 \pm 4$ & $<2$ & - & - & - & - & $23 \pm 7$ & $30 \pm 10$ & $30 \pm 10$ & - & - & - \\
\hline 1072 & 326.7 & $6 \pm 4$ & $<2$ & - & - & - & - & $30 \pm 10$ & $10 \pm 5$ & $10 \pm 10$ & - & - & - \\
\hline 1133 & 345.3 & $10 \pm 5$ & $<2$ & - & - & - & - & $23 \pm 7$ & $30 \pm 10$ & $50 \pm 10$ & - & - & - \\
\hline 1178 & 359.1 & $3 \pm 2$ & $<2$ & - & - & - & - & $18 \pm 7$ & $30 \pm 10$ & $40 \pm 10$ & - & - & - \\
\hline 1234 & 376.1 & $6 \pm 4$ & - & - & - & - & - & $18 \pm 7$ & $30 \pm 10$ & $40 \pm 10$ & - & - & - \\
\hline 1281 & 390.4 & $10 \pm 5$ & - & - & - & - & - & $23 \pm 7$ & $23 \pm 7$ & $10 \pm 10$ & - & - & - \\
\hline 1331 & 405.7 & $10 \pm 5$ & - & - & - & - & - & $23 \pm 7$ & $30 \pm 10$ & $10 \pm 10$ & - & - & - \\
\hline 1382 & 421.2 & $10 \pm 5$ & $<2$ & - & - & - & - & $23 \pm 7$ & $23 \pm 7$ & $50 \pm 10$ & - & - & - \\
\hline 1420 & 432.8 & $10 \pm 5$ & - & - & - & - & - & $23 \pm 7$ & $30 \pm 10$ & $10 \pm 10$ & - & - & - \\
\hline
\end{tabular}


Core

\begin{tabular}{|c|c|c|c|c|c|c|c|c|c|c|c|c|c|}
\hline sample & $\begin{array}{l}\text { Dopth } \\
\text { (a) }\end{array}$ & smoctite & Mica & $\begin{array}{l}\text { clino- } \\
\text { ptilolite }\end{array}$ & $\begin{array}{l}\text { Morden- } \\
\text { ite }\end{array}$ & $\begin{array}{l}\text { Ana1- } \\
\text { cine }\end{array}$ & $\begin{array}{c}\text { Iridy- } \\
\text { mite }\end{array}$ & Quartz & $\begin{array}{l}\text { Cristo. } \\
\text { balite }\end{array}$ & $\begin{array}{l}\text { Alkali } \\
\text { reldspar }\end{array}$ & calcite & Glas: & othor \\
\hline \multicolumn{14}{|l|}{ USW $G-2$} \\
\hline 1461 & 145.3 & $10 \pm 5$ & - & - & - & - & - & $15 \pm 5$ & $10 \pm 10$ & $40 \pm 10$ & - & - & - \\
\hline 1536 & 168.2 & $15 \pm 5$ & - & $3 \pm 2$ & - & - & - & $15 \pm 5$ & $40 \pm 10$ & $10 \pm 10$ & - & - & - \\
\hline 1585 & 483.1 & $10 \pm 5$ & - & $3 \pm 2$ & - & - & - & $18 \pm 7$ & $30 \pm 10$ & $40 \pm 10$ & - & - & - \\
\hline 1634 & 498.0 & $10 \pm 10$ & $<2$ & $40 \pm 10$ & - & - & - & - & $23 \pm 7$ & - & - & - & - \\
\hline 1664 & 507.2 & $<2$ & - & $<\overline{2}$ & - & - & - & $7 \pm 3$ & $7 \pm 3$ & $15 \pm 5$ & - & $60 \pm 10$ & - \\
\hline 1691 & 515.4 & $10 \pm 5$ & - & $50 \pm 10$ & $7 \pm 3$ & - & - & - & $20 \pm 5$ & $10 \pm 5$ & - & - & - \\
\hline 1745 & 531.9 & $15 \pm 5$ & - & $10_{ \pm 10}^{-10}$ & $7 \pm 3$ & - & - & $7 \pm^{3}$ & $23 \pm 7$ & $15 \pm 5$ & - & - & - \\
\hline 1752 & 534.0 & $10 \pm 5$ & - & $60 \pm 10$ & $7 \pm 3$ & - & - & $3 \pm 2$ & $10 \pm 5$ & $7 \pm 3$ & - & - & - \\
\hline 1798 & 548.0 & $<2$ & - & $50 \pm 10$ & $23 \pm 7$ & - & - & $10 \pm 5$ & $15 \pm 5$ & $23 \pm 7$ & - & - & - \\
\hline 1848 & 563.3 & - & - & $50 \pm 10$ & $23 \pm 7$ & - & - & $T r$ & $23 \pm 7$ & $15 \pm 5$ & - & - & - \\
\hline 1899 & 578.8 & $<2$ & $<2$ & $50 \pm 10$ & $23 \pm 7$ & - & - & $7 \pm 3$ & $15 \pm 5$ & $15 \pm 5$ & - & - & - \\
\hline 1952 & 595.0 & - & $3 \pm 2$ & $7 \pm 3$ & $50 \pm 10$ & - & - & $15 \pm 5$ & $15 \pm 5$ & $15 \pm 5$ & - & - & - \\
\hline 2001 & 609.9 & - & $<2$ & $7 \pm 3$ & $50 \pm 10$ & - & - & $15 \pm 5$ & $15 \pm 5$ & $23 \pm 7$ & - & - & - \\
\hline 2078 & 633.4 & $3 \pm 2$ & $3 \pm 2$ & $30 \pm 10$ & $30 \pm 10$ & - & - & $7 \pm 3$ & $15 \pm 5$ & $15 \pm 5$ & - & - & - \\
\hline 2158 & 657.8 & $3 \pm 2$ & $3 \pm 2$ & $30 \pm 10$ & $23 \pm 7$ & Tr. & - & $7 \pm 3$ & $23 \pm 7$ & $15 \pm 5$ & - & - & - \\
\hline 2248 & 685.2 & $3 \pm 2$ & $<2$ & $30 \pm 10$ & $30 \pm 10$ & - & - & $10 \pm 5$ & $23 \pm 7$ & $23 \pm 7$ & - & - & - \\
\hline 2353 & 717.2 & $\sqrt{2}$ & $6 \pm 4$ & $10 \pm 5$ & $30 \pm 10$ & - & - & $23 \pm 7$ & $23 \pm 7$ & $15 \pm 5$ & - & - & - \\
\hline 2430 & 740.7 & $3 \pm 2$ & $10 \pm 5$ & $30 \pm 10$ & $10 \pm 5$ & - & - & $30 \pm 10$ & $5 \pm 5$ & $30 \pm 20$ & - & - & - \\
\hline 2528 & 770.5 & $3 \pm 2$ & $10 \pm 5$ & $3 \pm 2$ & $23 \pm 7$ & - & - & $30 \pm 10$ & $3 \pm 2$ & $30 \pm 10$ & - & - & - \\
\hline 2667 & 812.9 & $3 \pm 2$ & $7 \pm 3$ & $=$ & $30 \pm 10$ & - & - & $23 \pm 7$ & $3 \pm 2$ & $10 \pm 10$ & - & - & - \\
\hline 2744 & 836.4 & $3 \pm 2$ & $<2$ & - & - & - & - & $10 \pm 10$ & $7 \pm 3$ & $50 \pm 10$ & - & - & - \\
\hline 2820 & 859.5 & $<\overline{2}$ & $<2$ & - & - & - & - & $30 \pm 10$ & $6 \pm 4$ & $60 \pm 10$ & - & - & - \\
\hline 2869 & 874.5 & $<2$ & $3 \pm 2$ & - & - & - & - & $30 \pm 10$ & $3 \pm 2$ & $60 \pm 10$ & - & - & - \\
\hline 2887 & 880.0 & $3 \pm 2$ & $<2$ & - & - & - & - & $30 \pm 10$ & $3 \pm 2$ & $60 \pm 10$ & - & - & - \\
\hline 2950 & 899.2 & $6 \pm 4$ & $<3$ & - & - & - & - & $30 \pm 10$ & $6 \pm 4$ & $50 \pm 10$ & - & - & - \\
\hline 2970 & 905.3 & $10 \pm 5$ & $<3$ & - & - & - & - & $40 \pm 10$ & $s_{ \pm 5}$ & $50 \pm 10$ & - & - & - \\
\hline 3037 & 925.7 & $10 \pm 10$ & $<2$ & - & $3 \pm 2$ & - & - & $30 \pm 13$ & $3 \pm 2$ & $23 \pm 7$ & - & - & - \\
\hline 3067 & 934.8 & $=$ & ‘2 & $7 \pm 3$ & $30 \pm 10$ & - & - & $=$ & $30 \pm 10$ & $30 \pm 10$ & - & - & - \\
\hline 3192 & 972.9 & $7 \pm 3$ & - & $30 \pm 10$ & $10 \pm 5$ & Ir. & - & $30 \pm 10$ & $3 \pm 2$ & $30 \pm 10$ & - & - & - \\
\hline 3228 & 983.9 & $6 \pm 3$ & - & $1 \pm 1$ & $=$ & $30 \pm 10$ & - & $34 \pm 10$ & $=$ & $29 \pm 10$ & - & - & - \\
\hline 3250 & 990.6 & $23 \pm 7$ & $<2$ & $10 \pm 5$ & $10 \pm 5$ & $30 \pm 10$ & - & $23 \pm 7$ & $3 \pm 2$ & $23 \pm 7$ & - & - & - \\
\hline 3308 & 1008.3 & $3 \pm 2$ & $15 \pm 5$ & $=$ & $=$ & $=$ & - & $23 \pm 7$ & $5 \pm 5$ & $50 \pm 10$ & - & - & - \\
\hline \multirow[t]{2}{*}{3330} & 1015.0 & - & $6 \pm 4$ & - & - & - & - & $30 \pm 10$ & $5 \pm 5$ & $60 \pm 10$ & - & - & - \\
\hline & Fracture & $10 \pm 5$ & $6 \pm 4$ & - & - & - & - & $15 \pm 5$ & - & $23 \pm 7$ & - & - & $50 \pm 10^{\circ}$ \\
\hline 3349 & 1020.8 & $<2$ & $6 \pm 4$ & - & - & - & - & $23 \pm 7$ & $5 \pm 5$ & $60 \pm 20$ & - & - & - \\
\hline
\end{tabular}




\begin{tabular}{|c|c|c|c|c|c|c|c|c|c|c|c|c|c|}
\hline Sample & $\begin{array}{l}\text { Depth } \\
\text { (1) }\end{array}$ & saectite & Mice & $\begin{array}{l}\text { Clino- } \\
\text { ptilolite }\end{array}$ & $\begin{array}{l}\text { Morden- } \\
\text { ite }\end{array}$ & $\begin{array}{l}\text { Anal- } \\
\text { Cine }\end{array}$ & $\begin{array}{l}\text { Tridy- } \\
\text { mite }\end{array}$ & Quartz & $\begin{array}{l}\text { Cristo- } \\
\text { balite }\end{array}$ & $\begin{array}{l}\text { Alkali } \\
\text { reldspar }\end{array}$ & calcite & Glass & other \\
\hline \multicolumn{14}{|l|}{ USW G-2 } \\
\hline 3366 & 1026.0 & $<2$ & $6 \pm 4$ & - & - & - & - & $30 \pm 10$ & $5 \pm 5$ & $60 \pm 10$ & - & - & - \\
\hline 3416 & 1041.2 & $<2$ & $6 \pm 4$ & - & - & - & - & $30 \pm 10$ & $=$ & $60 \pm 10$ & - & - & - \\
\hline 3454 & 1052.8 & $28 \pm 7$ & $3 \pm 2$ & - & $23 \pm 7$ & $\mathbf{T r}$. & - & $25 \pm 10$ & - & $25 \pm 10$ & - & - & - \\
\hline 3492 & 1064.4 & $18 \pm 7$ & $3 \pm 2$ & - & $23 \pm 7$ & $7 \pm 3$ & - & $30 \pm 10$ & - & $23 \pm 7$ & - & - & - \\
\hline 3512 & 1070.5 & $15 \pm 5$ & $7 \pm 3$ & - & $6 \pm 4$ & $15 \pm 5$ & - & $30 \pm 10$ & - & $10 \pm 10$ & - & - & $3 \pm 2^{b}$ \\
\hline 3541 & 1079.3 & $10 \pm 5$ & $7 \pm 3$ & - & - & $30 \pm 10$ & - & $30 \pm 10$ & - & $23 \pm 7$ & - & - & $3 \pm 2^{b}$ \\
\hline 3578 & 1090.6 & $23 \pm 7$ & $10 \pm 5$ & - & $23 \pm 7$ & $=$ & - & $30 \pm 10$ & - & $23 \pm 7$ & - & - & \pm \\
\hline 3627 & 1105.5 & $15 \pm 5$ & $10 \pm 5$ & - & - & $3 \pm 2$ & - & $30 \pm 10$ & - & $40 \pm 10$ & - & - & - \\
\hline 3671 & 1118.9 & $10 \pm 5$ & $7 \pm 3$ & - & - & $7 \pm 3$ & - & $30 \pm 10$ & - & $40 \pm 10$ & $7 \pm 3$ & - & $<2^{b}$ \\
\hline 3720 & 1133.9 & $10 \pm 5$ & $7 \pm 3$ & - & - & $\mathbf{T r}$ & - & $30 \pm 10$ & - & $40 \pm 10$ & $\overline{-}$ & - & - \\
\hline 3724 & 1135.1 & $25 \pm 5$ & $3 \pm 2$ & - & - & - & - & $10 \pm 10$ & - & $30 \pm 10$ & - & - & $3 \pm 2^{b}$ \\
\hline 3750 & 1143.0 & $25 \pm 5$ & $10 \pm 5$ & - & - & - & - & $30 \pm 10$ & - & $10 \pm 10$ & - & - & - \\
\hline 3772 & 1149.7 & $23 \pm 7$ & $7 \pm 3$ & - & - & - & - & $30 \pm 10$ & - & $10 \pm 10$ & - & - & - \\
\hline 3795 & 1156.7 & $32 \pm 8$ & - & - & - & $<2$ & - & $10 \pm 10$ & - & $30 \pm 10$ & - & - & $3 \pm 2^{b}$ \\
\hline 3833 & 1168.3 & $30 \pm 10$ & $6 \pm 4$ & - & - & - & - & $30 \pm 10$ & - & $30 \pm 10$ & - & - & - \\
\hline 3875 & 1181.1 & $25 \pm 5$ & $7 \pm 3$ & - & - & $3 \pm 2$ & - & $40 \pm 10$ & - & $23 \pm 7$ & - & - & - \\
\hline 3908 & 1191.2 & $18 \pm 7$ & 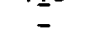 & - & - & - & $\rightarrow$ & $30 \pm 10$ & - & $40 \pm 10$ & $6 \pm 4$ & - & - \\
\hline 3933 & 1198.0 & $15 \pm 5$ & - & - & - & $30 \pm 10$ & - & $30 \pm 10$ & - & $30 \pm 10$ & $7 \pm 3$ & - & - \\
\hline 3968 & 1209.4 & $18 \pm 7$ & $6 \pm 4$ & - & - & $\mathrm{Tr}$ & - & $40 \pm 10$ & - & $40 \pm 10$ & - & - & $T r . b$ \\
\hline 4005 & 1220.7 & $15 \pm 5$ & $10 \pm 5$ & - & - & $3 \pm 2$ & - & $23 \pm 7$ & - & $10 \pm 10$ & $7 \pm 3$ & - & - \\
\hline $\begin{array}{l}4090 \\
4167\end{array}$ & $\begin{array}{l}1246.6 \\
1270.1\end{array}$ & $\begin{array}{l}23 \pm 7 \\
15+5\end{array}$ & $\begin{array}{l}18 \pm 7 \\
15+5\end{array}$ & $=$ & - & $\begin{array}{l}6 \pm 4 \\
<2\end{array}$ & - & $\begin{array}{l}23 \pm 7 \\
23+7\end{array}$ & - & $\begin{array}{l}30 \pm 10 \\
10+10\end{array}$ & $\begin{array}{l}10 \pm 5 \\
10+5\end{array}$ & & $-\overline{3}+2^{b}$ \\
\hline $\begin{array}{l}4167 \\
1199\end{array}$ & $\begin{array}{l}1270.1 \\
1279.9\end{array}$ & $\begin{array}{l}15 \pm 5 \\
15 \pm 5\end{array}$ & $\begin{array}{l}15 \pm 5 \\
10 \pm 5\end{array}$ & 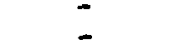 & - & $\begin{array}{l}<2 \\
\mathrm{Tr} .\end{array}$ & $\overline{-}$ & $\begin{array}{l}23 \pm 7 \\
18 \pm 7\end{array}$ & - & $\begin{array}{l}10 \pm 10 \\
10 \pm 10\end{array}$ & $\begin{array}{l}10 \pm 5 \\
18 \pm 7\end{array}$ & - & $\begin{array}{l}3 \pm 2^{\circ} \\
<2^{b}\end{array}$ \\
\hline 4209 & 1282.9 & $10 \pm 5$ & $7 \pm 3$ & - & - & $6 \pm 4$ & - & $18 \pm 7$ & - & $50 \pm 10$ & $3 \pm 2$ & - & - \\
\hline 1267 & 1300.6 & $15 \pm 5$ & $\overrightarrow{7 \pm 3}$ & - & - & $3 \pm 2$ & - & $30 \pm 10$ & - & $10 \pm 10$ & $3 \pm 2$ & - & - \\
\hline 4329 & 1319.5 & $10 \pm 5$ & $6 \pm 4$ & - & - & $3 \pm 2$ & - & $30 \pm 10$ & - & $10 \pm 10$ & $10 \pm 5$ & - & - \\
\hline 4467 & 1361.5 & $10 \pm 5$ & $6 \pm 4$ & - & - & $3 \pm 2$ & - & $23 \pm 7$ & - & $10 \pm 10$ & $10 \pm 5$ & - & - \\
\hline & Punice & $23 \pm 7$ & $=$ & - & - & - & - & $23 \pm 7$ & - & $10 \pm 10$ & $10 \pm 5$ & - & - \\
\hline 4570 & 1392.9 & $10 \pm 5$ & $7 \pm 3$ & - & - & $3 \pm 2$ & - & $30 \pm 10$ & - & $10 \pm 10$ & $18 \pm 7$ & - & - \\
\hline 4788 & 1459.4 & $15 \pm 5$ & $3 \pm 2$ & - & - & $\mathbf{T r}$ & - & $30 \pm 10$ & - & $10 \pm 10$ & $10 \pm 5$ & - & - \\
\hline 4805 & 1464.6 & $20 \pm 10$ & - & - & - & $\mathbf{T r}$ & - & $40 \pm 10$ & - & $40 \pm 10$ & - & - & - \\
\hline 4816 & 1467.9 & $23 \pm 7$ & - & - & - & Tr. & - & $40 \pm 10$ & - & $30 \pm 10$ & $6 \pm 4$ & - & - \\
\hline 1838 & 1474.6 & $23 \pm 7$ & - & - & - & $3 \pm 2$ & - & $30 \pm 10$ & - & $40 \pm 10$ & $6 \pm 4$ & - & - \\
\hline 4873 & 1485.3 & $30 \pm 10$ & - & - & - & $<\overline{2}$ & - & $30 \pm 10$ & - & $30 \pm 10$ & $=$ & - & - \\
\hline 4885 & 1488.9 & $30 \pm 10$ & - & - & - & - & - & $30 \pm 10$ & - & $30 \pm 10$ & $3 \pm 2$ & - & - \\
\hline
\end{tabular}




\begin{tabular}{|c|c|c|c|c|c|c|c|c|c|c|c|c|c|}
\hline Sample & $\begin{array}{l}\text { Dopth } \\
(m)\end{array}$ & snoctite & Mica & $\begin{array}{l}\text { Clino- } \\
\text { ptilolite }\end{array}$ & $\begin{array}{l}\text { Morden- } \\
\text { ite }\end{array}$ & $\begin{array}{l}\text { Anal- } \\
\text { cine }\end{array}$ & $\begin{array}{l}\text { Tridy- } \\
\text { mite }\end{array}$ & Quartz & $\begin{array}{l}\text { Cristo- } \\
\text { balite }\end{array}$ & $\begin{array}{l}\text { Alkali } \\
\text { Foldspar }\end{array}$ & calcite & Glass & other \\
\hline \multicolumn{14}{|c|}{ USW G-2 } \\
\hline 4893 & 1491.4 & $23 \pm 7$ & $7 \pm 3$ & - & - & $<2$ & - & $30 \pm 10$ & - & $30 \pm 10$ & - & - & - \\
\hline 1924 & 1500.8 & $18 \pm 7$ & $10 \pm 5$ & - & - & $3 \pm 2$ & - & $30 \pm 10$ & - & $30 \pm 10$ & $7 \pm 3$ & - & - \\
\hline 1949 & 1508.5 & $10 \pm 10$ & $10 \pm 5$ & - & - & $=$ & - & $23 \pm 7$ & - & $23 \pm 7$ & - & - & - \\
\hline \multirow[t]{2}{*}{5017} & 1529.2 & $20 \pm 10$ & $=$ & - & - & - & - & $30 \pm 10$ & - & $40 \pm 10$ & - & - & $3 \pm 2^{b}$ \\
\hline & Spherulite & $6 \pm 4$ & - & - & - & - & - & $40 \pm 10$ & - & $40 \pm 10$ & $6 \pm 4$ & - & $3 \pm 2^{b}$ \\
\hline 5029 & 1532.8 & $20 \pm 10$ & $20 \pm 10$ & - & - & - & - & $30 \pm 10$ & - & $30 \pm 10$ & - & - & - \\
\hline 5144 & 1567.9 & $6 \pm 4$ & $20 \pm 10$ & - & - & - & - & $30 \pm 10$ & - & $40 \pm 10$ & $6 \pm 4$ & - & - \\
\hline 5171 & 1576.1 & $28 \pm 7$ & $30 \pm 10$ & - & - & - & - & $23 \pm 7$ & - & $23 \pm 7$ & $6 \pm 4$ & - & $-b$ \\
\hline 5206 & 1586.8 & $15 \pm 5$ & $18 \pm 7$ & - & - & Tr. & - & $23 \pm 7$ & - & $40 \pm 10$ & - & - & $6 \pm 4^{b}$ \\
\hline 5213 & 1588.9 & $10 \pm 5$ & $10 \pm 5$ & - & - & - & - & $30 \pm 10$ & - & $30 \pm 10$ & $6 \pm 4$ & - & $10 \pm 5^{b}$ \\
\hline 5305 & 1617.0 & $10 \pm 5$ & $10 \pm 5$ & - & - & - & - & $23 \pm 7$ & - & $40 \pm 10$ & $10 \pm 5$ & - & $<2^{b}$ \\
\hline 5369 & 1636.5 & $10 \pm 5$ & $15 \pm 5$ & - & - & - & - & $30 \pm 10$ & - & $40 \pm 10$ & $10 \pm 5$ & - & - \\
\hline 5379 & 1639.5 & $6 \pm 4$ & $15 \pm 5$ & - & - & - & - & $30 \pm 10$ & - & $10 \pm 10$ & $10 \pm 5$ & - & - \\
\hline 5434 & 1656.3 & $6 \pm 4$ & $23 \pm 7$ & - & - & $<2$ & - & $23 \pm 7$ & - & $40 \pm 10$ & - & - & - \\
\hline 5493 & 1674.3 & $3 \pm 2$ & $23 \pm 7$ & - & - & - & - & $30 \pm 10$ & - & $40 \pm 10$ & $15 \pm 5$ & - & - \\
\hline 5505 & 1677.9 & $10 \pm 5$ & $15 \pm 5$ & - & - & - & - & $23 \pm 7$ & - & $40 \pm 10$ & $7 \pm 3$ & $\therefore \quad-$ & $6 \pm 4^{c}$ \\
\hline 5538 & 1688.0 & $25 \pm 10$ & $=$ & - & - & - & - & $23 \pm 7$ & - & $30 \pm 10$ & $18 \pm 7$ & - & $10 \pm 5^{c}$ \\
\hline 5596 & 1705.7 & $15 \pm 5$ & - & - & - & - & - & $23 \pm 7$ & - & $10 \pm 10$ & $6 \pm 4$ & - & $7 \pm 3^{c}$ \\
\hline 5638 & 1718.5 & $18 \pm 7$ & - & - & - & $T x$ & - & $18 \pm 7$ & - & $40 \pm 10$ & $18 \pm 7$ & - & $6 \pm 4^{c}$ \\
\hline 5657 & 1724.3 & $30 \pm 10$ & - & - & - & - & - & $23 \pm 7$ & - & $30 \pm 10$ & $6 \pm 4$ & - & $6 \pm 4^{c}$ \\
\hline 5696 & 1736.1 & $15 \pm 5$ & - & - & - & $<2$ & - & $23 \pm 7$ & - & $40 \pm 10$ & $18 \pm 7$ & - & $10 \pm 5^{c}$ \\
\hline 5762 & 1756.3 & $10 \pm 5$ & - & - & - & - & - & $23 \pm 7$ & - & $40 \pm 10$ & $23 \pm 7$ & - & $10 \pm 5^{c}$ \\
\hline 5820 & 1773.9 & - & - & - & - & - & - & $23 \pm 7$ & - & $10 \pm 10$ & $6 \pm 4$ & - & $18 \pm 7^{c}$ \\
\hline 5885 & 1793.7 & $18 \pm 7$ & - & - & - & - & - & $23 \pm 7$ & - & $18 \pm 7$ & $30 \pm 10$ & - & $6 \pm 4^{c}$ \\
\hline 5895 & 1796.8 & $18 \pm 7$ & - & - & - & - & - & $30 \pm 10$ & - & $30 \pm 10$ & $23 \pm 7$ & - & $6 \pm 4^{c}$ \\
\hline 5918 & 1803.8 & $10 \pm 5$ & - & - & - & - & - & $10 \pm 10$ & - & $40 \pm 10$ & - & - & $6 \pm 4^{b}, 3 \pm 2^{c}$ \\
\hline 5926 & 1806.2 & $15 \pm 5$ & - & - & - & Tx. & - & $10 \pm 10$ & - & $40 \pm 10$ & - & - & - \\
\hline 5931 & 1807.8 & $23 \pm 7$ & - & - & - & - & - & $23 \pm 7$ & - & $10 \pm 10$ & $6 \pm 4$ & - & $10 \pm 5^{b}$ \\
\hline 5951 & 1813.9 & $23 \pm 7$ & - & - & - & $<2$ & - & $30 \pm 10$ & - & $40 \pm 10$ & $6 \pm 4$ & - & $3 \pm 2^{b}$ \\
\hline 5971 & 1820.0 & $18 \pm 7$ & - & - & - & $3 \pm 2$ & - & $30 \pm 10$ & - & $10 \pm 10$ & - & - & - \\
\hline 5997 & 1826.4 & $18 \pm 7$ & - & - & - & $3 \pm 2$ & - & $40 \pm 10$ & - & $10 \pm 10$ & $6 \pm 4$ & - & $3 \pm 2^{b}$ \\
\hline
\end{tabular}


APPENDIX A (cont)

Core

\begin{tabular}{|c|c|c|c|c|c|c|c|c|c|c|c|c|}
\hline Sanple & $\begin{array}{l}\text { Depth } \\
\text { (a) }\end{array}$ & smectite & Mica & $\begin{array}{c}\text { Clino- } \\
\text { ptilolite }\end{array}$ & $\begin{array}{l}\text { Anal- } \\
\text { cime }\end{array}$ & $\begin{array}{l}\text { Tridy- } \\
\text { nite }\end{array}$ & Quartz & $\begin{array}{l}\text { Cristo- } \\
\text { balite }\end{array}$ & $\begin{array}{l}\text { Alkali } \\
\text { Foldspar }\end{array}$ & calcite & Glass & other \\
\hline
\end{tabular}

USW GU-3

\begin{tabular}{|c|c|c|c|c|c|c|c|c|c|c|c|c|}
\hline 31.0 & 9.5 & $3 \pm 1$ & $2 \pm 1$ & - & - & - & - & $10 \pm 5$ & $75 \pm 5$ & $12 \pm 3$ & - & - \\
\hline 45.0 & 13.7 & - & - & - & - & $6 \pm 2$ & - & $17 \pm 3$ & $75 \pm 5$ & - & - & - \\
\hline 79.0 & 24.1 & - & - & - & - & $25 \pm 5$ & $2 \pm 1$ & $6 \pm 2$ & $70 \pm 5$ & - & - & - \\
\hline \multirow[t]{2}{*}{103.1} & 31.4 & - & - & - & - & $7 \pm 3$ & $2 \pm 1$ & $20 \pm 5$ & $70 \pm 5$ & $2 \pm 1$ & - & - \\
\hline & Eracture & - & - & - & - & $2 \pm 1$ & - & $2 \pm 1$ & $9 \pm 3$ & $90 \pm 5$ & - & - \\
\hline \multirow[t]{2}{*}{196.3} & 59.8 & - & - & - & - & - & - & $25 \pm 5$ & $75 \pm 5$ & - & - & - \\
\hline & vein & $3 \pm 1$ & - & - & - & - & - & $3 \pm 2$ & $10 \pm 5$ & $85 \pm 5$ & - & - \\
\hline 245.7 & $\overline{74.9}$ & $2 \pm 1$ & - & - & - & $3 \pm 1$ & $2 \pm 1$ & $25 \pm 5$ & $70 \pm 5$ & - & - & - \\
\hline 303.6 & 92.5 & $<1$ & - & - & - & - & - & $25 \pm 5$ & $75 \pm 5$ & - & - & - \\
\hline 316.8 & 96.6 & - & - & - & - & - & - & $22 \pm 3$ & $75 \pm 5$ & - & - & - \\
\hline 341.5 & 104.1 & $3 \pm 2$ & - & - & - & - & - & $30 \pm 5$ & $70 \pm 5$ & - & - & - \\
\hline 356.5 & 108.7 & $7 \pm 3$ & - & - & - & - & - & $15 \pm 5$ & $40 \pm 5$ & - & $10 \pm 10$ & - \\
\hline 376.1 & 114.6 & $4 \pm 2$ & - & - & - & - & $3 \pm 1$ & $5 \pm 3$ & $50 \pm 5$ & - & $40 \pm 10$ & - \\
\hline 410.0 & 125.0 & $20 \pm 4$ & -1 & - & - & - & $2 \pm 1$ & $25 \pm 5$ & $50 \pm 5$ & - & - & - \\
\hline 414.3 & 126.3 & $3 \pm 2$ & -1 & - & - & - & $3 \pm 2$ & $20 \pm 5$ & $75 \pm 5$ & - & - & - \\
\hline 417.5 & 127.3 & $12 \pm 3$ & -1 & - & - & - & $6 \pm 2$ & $17 \pm 3$ & $65 \pm 5$ & - & - & - \\
\hline 424.4 & 129.4 & - & $\sim 1$ & - & - & $6 \pm 2$ & $2 \pm 1$ & $17 \pm 3$ & $75 \pm 5$ & - & - & - \\
\hline \multirow[t]{2}{*}{429.0} & 130.8 & - & $2 \pm 1$ & - & - & $4 \pm 2$ & $4 \pm 2$ & $20 \pm 5$ & $70 \pm 5$ & - & - & - \\
\hline & Vein & - & - & - & - & - & - & $2 \pm 1$ & $6 \pm 2$ & $92 \pm 3$ & - & - \\
\hline 430.5 & 131.2 & - & $3 \pm 1$ & - & - & $4 \pm 2$ & - & $12 \pm 3$ & $80 \pm 5$ & - & - & - \\
\hline 465.5 & 141.9 & - & -1 & - & - & $10 \pm 5$ & - & $9 \pm 3$ & $80 \pm 5$ & - & - & - \\
\hline 482.0 & 146.9 & - & -1 & - & - & $20 \pm 5$ & - & $7 \pm 3$ & $70 \pm 5$ & $3 \pm 1$ & - & - \\
\hline \multirow[t]{2}{*}{520.3} & 158.6 & - & - & - & - & $20 \pm 5$ & - & $5 \pm 3$ & $70 \pm 5$ & $3 \pm 1$ & - & - \\
\hline & Cavity & - & - & - & - & $15 \pm 5$ & - & - & $12 \pm 3$ & $77 \pm 3$ & - & - \\
\hline \multirow[t]{2}{*}{525.3} & 160.1 & -1 & - & - & - & $20 \pm 5$ & -1 & $6 \pm 2$ & $70 \pm 5$ & - & - & - \\
\hline & Cavity & - & - & - & - & $35 \pm 10$ & - & $2 \pm 1$ & $53 \pm 5$ & $10 \pm 5$ & - & - \\
\hline 579.0 & 176.5 & -1 & - & - & - & $12 \pm 3$ & - & $22 \pm 3$ & $65 \pm 5$ & - & - & - \\
\hline 633.4 & 193.1 & -1 & -1 & - & - & $7 \pm 3$ & $2 \pm 1$ & $22 \pm 3$ & $70 \pm 5$ & - & - & - \\
\hline \multirow[t]{2}{*}{674.7} & 205.7 & -1 & -1 & - & - & $5 \pm 3$ & $2 \pm 1$ & $22 \pm 3$ & $70 \pm 5$ & - & - & - \\
\hline & Feacture & - & - & - & - & $70 \pm 10$ & $12 \pm 3$ & - & $17 \pm 3$ & - & - & - \\
\hline 702.5 & 214.1 & -1 & -1 & - & - & - & $6 \pm 2$ & $17 \pm 3$ & $70 \pm 5$ & - & - & - \\
\hline
\end{tabular}




\begin{tabular}{|c|c|c|c|c|c|c|c|c|c|c|c|c|}
\hline sanple & $\begin{array}{l}\text { Dopth } \\
\text { (II) }\end{array}$ & suoctite & Mica & $\begin{array}{c}\text { Clino- } \\
\text { ptilolit. }\end{array}$ & $\begin{array}{l}\text { Anal- } \\
\text { cime }\end{array}$ & $\begin{array}{c}\text { Tridy- } \\
\text { nite }\end{array}$ & Quartz & $\begin{array}{l}\text { Cristo- } \\
\text { balite }\end{array}$ & $\begin{array}{c}\text { Alkali } \\
\text { Foldspar }\end{array}$ & calcite & Glass & other \\
\hline \multicolumn{13}{|l|}{ USW GU-3 } \\
\hline \multirow{3}{*}{$\begin{array}{l}769.1 \\
849.4\end{array}$} & 234.4 & -1 & -1 & - & - & $6 \pm 4$ & -1 & $22 \pm 3$ & $65 \pm 5$ & - & - & - \\
\hline & 258.9 & -1 & -1 & - & - & $=$ & $4 \pm 2$ & $17 \pm 3$ & $75 \pm 5$ & - & - & - \\
\hline & Eracture & - & - & - & - & $5 \pm 3$ & $77 \pm 3$ & $-\overline{1}$ & $15 \pm 5$ & - & - & - \\
\hline \multirow[t]{2}{*}{910.5} & 277.5 & -1 & - & - & - & - & $4 \pm 2$ & $27 \pm 3$ & $65 \pm 5$ & - & - & - \\
\hline & rracture & -1 & - & - & - & $40 \pm 10$ & $17 \pm 3$ & $2 \pm 1$ & $40 \pm 5$ & - & - & - \\
\hline \multirow[t]{2}{*}{924.3} & 281.7 & -1 & - & - & - & $10 \pm 5$ & $12 \pm 3$ & $12 \pm 3$ & $65 \pm 5$ & - & - & - \\
\hline & Fracture & - & - & - & - & $12 \pm 3$ & $80 \pm 5$ & $-\overline{1}$ & $7 \pm 3$ & - & - & - \\
\hline \multirow{2}{*}{951.1} & 289.9 & $\sim 1$ & - & - & - & $5 \pm 3$ & $8 \pm 2$ & $17 \pm 3$ & $70 \pm 5$ & - & - & - \\
\hline & racture & - & - & - & - & $25 \pm 5$ & $50 \pm 5$ & $2 \pm 1$ & $24 \pm 5$ & - & - & - \\
\hline 954.8 & 291.0 & -1 & -1 & - & - & $=$ & $17 \pm 3$ & $12 \pm 3$ & $70 \pm 5$ & - & - & - \\
\hline \multirow[t]{2}{*}{1027.0} & 313.0 & - & - & - & - & - & $6 \pm 4$ & $17 \pm 3$ & $75 \pm 5$ & - & - & - \\
\hline & Fracture & $2 \pm 1$ & - & - & - & - & $7 \pm 2$ & $-\overline{1}$ & $6 \pm 2$ & $40 \pm 5$ & - & $40 \pm 5^{a}$ \\
\hline 1061.0 & 323.4 & - & - & - & - & - & $20 \pm 2$ & $7 \pm 3$ & $70 \pm 5$ & - & - & - \\
\hline 1130.3 & 344.5 & - & -1 & - & - & - & $17 \pm 3$ & $10 \pm 2$ & $70 \pm 5$ & - & - & - \\
\hline 1175.0 & 358.1 & -1 & -1 & - & - & - & $35 \pm 5$ & $3 \pm 1$ & $60 \pm 5$ & - & - & - \\
\hline \multirow[t]{2}{*}{1195.7} & 364.5 & $5 \pm 1$ & - & - & - & - & $3 \pm 1$ & $27 \pm 3$ & $35 \pm 5$ & - & $30 \pm 10$ & - \\
\hline & $\underline{v} \bullet \mathbf{i n}$ & $=$ & $\mathbf{T r}$. & -1 & - & - & $8 \pm 2$ & $22 \pm 3$ & $70 \pm 5$ & - & - & - \\
\hline 1227.0 & $3 \overline{74.0}$ & $2 \pm 1$ & - & - & - & - & $8 \pm 2$ & $17 \pm 3$ & $35 \pm 5$ & - & $40 \pm 10$ & - \\
\hline 1302.4 & 397.0 & $2 \pm 1$ & - & - & - & - & $4 \pm 2$ & $12 \pm 3$ & $40 \pm 5$ & - & $45 \pm 15$ & - \\
\hline 1322.0 & 403.0 & - & - & - & - & - & $4 \pm 2$ & $7 \pm 3$ & $30 \pm 5$ & - & $65 \pm 15$ & - \\
\hline 1344.8 & 409.9 & - & - & - & - & - & $7 \pm 3$ & $7 \pm 3$ & $30 \pm 5$ & - & $55 \pm 15$ & - \\
\hline 1369.6 & 417.5 & - & - & - & - & - & $4 \pm 2$ & $6 \pm 4$ & $25 \pm 5$ & - & $65 \pm 15$ & - \\
\hline 1394.5 & 425.0 & - & - & - & - & - & $1 \pm 2$ & $6 \pm 4$ & $30 \pm 5$ & - & $65 \pm 15$ & - \\
\hline 1394.6 & 425.1 & - & - & - & - & - & $4 \pm 2$ & $6 \pm 4$ & $30 \pm 5$ & - & $65 \pm 15$ & - \\
\hline 1415.5 & 431.4 & - & - & - & - & - & $5 \pm 3$ & $6 \pm 4$ & $35 \pm 5$ & - & $55 \pm 15$ & - \\
\hline 1439.2 & 438.7 & $\mathbf{T r}$ & $\mathbf{T r}$. & - & - & - & $20 \pm 5$ & $6 \pm 4$ & $20 \pm 5$ & - & $55 \pm 15$ & - \\
\hline 1439.5 & 438.8 & -1 & $2 \pm 1$ & - & - & - & $5 \pm 3$ & $6 \pm 4$ & $35 \pm 5$ & - & $55 \pm 15$ & - \\
\hline 1468.5 & 447.6 & - & $2 \pm 1$ & - & - & - & $5 \pm 3$ & $6 \pm 4$ & $35 \pm 5$ & - & $55 \pm 15$ & - \\
\hline 1493.7 & 455.3 & -1 & - & $2 \pm 1$ & - & - & $5 \pm 3$ & $6 \pm 4$ & $25 \pm 5$ & - & $65 \pm 15$ & - \\
\hline 1498.3 & 456.7 & - & - & $3 \pm 1$ & - & - & $7 \pm 3$ & $6 \pm 4$ & $25 \pm 5$ & - & $65 \pm 15$ & - \\
\hline $\begin{array}{l}1537.5 \\
1571.6\end{array}$ & $\begin{array}{l}468.6 \\
479.0\end{array}$ & $3 \pm 1$ & $3 \pm 1$ & $2 \pm 1$ & - & - & $17 \pm 3$ & $4 \pm 2$ & $50 \pm 5$ & $3 \pm 1$ & $20 \pm 10$ & $2 \pm 1^{b}$ \\
\hline 1571.6 & 479.0 & $2 \pm 1$ & $2 \pm 1$ & - & - & - & $4 \pm 2$ & $5 \pm 3$ & $45 \pm 5$ & - & $45 \pm 15$ & - \\
\hline 1598.5 & 487.2 & $4 \pm 2$ & -1 & - & - & - & $5 \pm 3$ & $2 \pm 1$ & $45 \pm 5$ & - & $45 \pm 15$ & - \\
\hline 1603.0 & 488.6 & - & -1 & - & - & - & $2 \pm 1$ & $30 \pm 5$ & $65 \pm 5$ & - & - & - \\
\hline
\end{tabular}


APPENDIX A (cont)

core

\begin{tabular}{|c|c|c|c|c|c|c|c|c|c|c|c|c|}
\hline sanple & $\begin{array}{c}\text { Depth } \\
(\mathbb{m})\end{array}$ & Smectite & Mica & $\begin{array}{l}\text { Clino- } \\
\text { ptilolite }\end{array}$ & $\begin{array}{l}\text { Anal- } \\
\text { cias }\end{array}$ & $\begin{array}{l}\text { Tridy- } \\
\text { mite }\end{array}$ & Quartz & $\begin{array}{l}\text { Cristo- } \\
\text { balite }\end{array}$ & $\begin{array}{l}\text { Alkali } \\
\text { Feldspar }\end{array}$ & calcite & Glass & other \\
\hline USW GU-3 & & & & & & & & & & & & \\
\hline $\begin{array}{l}1624.2 \\
1653.2 \\
1709.0 \\
1744.0 \\
1827.2^{\mathrm{C}}\end{array}$ & $\begin{array}{l}495.1 \\
504.0 \\
521.0 \\
531.6 \\
557.0\end{array}$ & $\begin{array}{c}- \\
-1 \\
-1 \\
- \\
2 \pm 1\end{array}$ & $\begin{array}{l}-1 \\
-1 \\
- \\
- \\
-\end{array}$ & $\begin{array}{c}- \\
- \\
- \\
\overline{30 \pm 2}\end{array}$ & $\begin{array}{l}- \\
- \\
- \\
-\end{array}$ & $\begin{array}{c}7 \pm 3 \\
15 \pm 5 \\
- \\
- \\
-\end{array}$ & $\begin{array}{r}20 \pm 5 \\
15 \pm 5 \\
12 \pm 3 \\
6 \pm 2 \\
4 \pm 1\end{array}$ & $\begin{array}{r}2 \pm 1 \\
3 \pm 2 \\
12 \pm 3 \\
25 \pm 5 \\
7 \pm 2\end{array}$ & $\begin{array}{l}70 \pm 5 \\
70 \pm 5 \\
70 \pm 5 \\
70 \pm 5 \\
19 \pm 3\end{array}$ & $\begin{array}{c}- \\
- \\
-\end{array}$ & $\begin{array}{c}- \\
- \\
- \\
38 \pm 4\end{array}$ & $\begin{array}{l}- \\
- \\
- \\
-\end{array}$ \\
\hline $\begin{array}{l}1874.0 \\
1935.8 \\
1986.0 \\
1993.1 \\
2013.2\end{array}$ & $\begin{array}{r}571.2 \\
590.0 \\
605.3 \\
607.5 \\
613.6 \\
\text { Fracture } \\
\end{array}$ & $\begin{array}{c}- \\
3 \pm 2 \\
- \\
- \\
2 \pm 1 \\
30 \pm 5\end{array}$ & $\begin{array}{l}- \\
- \\
- \\
- \\
2 \pm 1 \\
3 \pm 1\end{array}$ & $\begin{array}{l}50 \pm 5 \\
60 \pm 5 \\
70 \pm 5 \\
60 \pm 5 \\
50 \pm 5 \\
15 \pm 5\end{array}$ & $\begin{array}{l}- \\
- \\
- \\
- \\
-\end{array}$ & $\begin{array}{l}- \\
\overline{-} \\
\overline{-}\end{array}$ & $\begin{array}{r}7 \pm 3 \\
4 \pm 2 \\
4 \pm 2 \\
4 \pm 2 \\
3 \pm 1 \\
10 \pm 5\end{array}$ & $\begin{array}{r}5 \pm 3 \\
4 \pm 2 \\
6 \pm 2 \\
10 \pm 2 \\
4 \pm 2 \\
-\end{array}$ & $\begin{array}{l}40 \pm 5 \\
30 \pm 5 \\
20 \pm 5 \\
25 \pm 5 \\
40 \pm 5 \\
40 \pm 5\end{array}$ & $\begin{array}{l}- \\
- \\
z \\
-\end{array}$ & $\begin{array}{l}- \\
- \\
- \\
-\end{array}$ & $\begin{array}{l}- \\
- \\
- \\
- \\
-\end{array}$ \\
\hline $\begin{array}{l}2070.2 \\
2138.2 \\
2177.3 \\
2189.3 \\
2198.0\end{array}$ & $\begin{array}{r}631.0 \\
651.7 \\
663.6 \\
667.3 \\
670.0 \\
\text { Fracture } \\
\end{array}$ & $\begin{array}{l}2 \pm 1 \\
2 \pm 1 \\
2 \pm 1 \\
2 \pm 1 \\
2 \pm 1 \\
7 \pm 2\end{array}$ & $\begin{array}{l}- \\
2 \pm 1 \\
2 \pm 1 \\
2 \pm 1 \\
2 \pm 1 \\
1 \pm 1\end{array}$ & $\begin{array}{l}- \\
- \\
- \\
- \\
2 \pm 1\end{array}$ & $\begin{array}{l}- \\
\overline{-} \\
\bar{z} \\
-\end{array}$ & $\begin{array}{c}7 \pm 3 \\
- \\
- \\
- \\
- \\
-\end{array}$ & $\begin{array}{l}20 \pm 5 \\
17 \pm 3 \\
20 \pm 4 \\
12 \pm 3 \\
12 \pm 3 \\
11 \pm 3\end{array}$ & $\begin{array}{r}3 \pm 1 \\
12 \pm 3 \\
10 \pm 4 \\
15 \pm 3 \\
17 \pm 3 \\
10 \pm 2\end{array}$ & $\begin{array}{l}70 \pm 5 \\
70 \pm 5 \\
65 \pm 5 \\
70 \pm 5 \\
70 \pm 5 \\
69 \pm 5\end{array}$ & $\begin{array}{l}- \\
- \\
- \\
-\end{array}$ & $\begin{array}{l}- \\
- \\
- \\
- \\
-\end{array}$ & $\begin{array}{c}-\bar{a} \\
\mathbf{T r}^{\mathbf{b}} \\
\mathbf{T r} \mathbf{r}^{\mathbf{b}} \\
\mathbf{T} \mathbf{r}^{\mathbf{b}} \\
- \\
-\end{array}$ \\
\hline $\begin{array}{l}2226.0 \\
2360.0 \\
2369.4\end{array}$ & $\begin{array}{r}678.5 \\
\text { Frecture } \\
719.3 \\
\text { Fracture } \\
722.2\end{array}$ & $\begin{array}{c}99 \pm 1 \\
- \\
2 \pm 1 \\
2 \pm 1\end{array}$ & $\begin{array}{l}- \\
2 \pm 1 \\
2 \pm 1 \\
2 \pm 1\end{array}$ & $\begin{array}{l}- \\
- \\
-\end{array}$ & $\begin{array}{l}- \\
- \\
-\end{array}$ & $\begin{array}{l}- \\
-\end{array}$ & $\begin{array}{c}- \\
20 \pm 3 \\
37 \pm 3 \\
17 \pm 3\end{array}$ & $\begin{array}{c}- \\
12 \pm 3 \\
12 \pm 3\end{array}$ & $\begin{array}{c}- \\
70 \pm 5 \\
10 \pm 5 \\
70 \pm 5\end{array}$ & $\begin{array}{l}- \\
- \\
-\end{array}$ & $\begin{array}{l}- \\
- \\
-\end{array}$ & $\begin{array}{r}1 \pm 1 d \\
1 \pm 1^{b} \\
17 \pm 8^{\circ} \\
1 \pm 1^{b}\end{array}$ \\
\hline $\begin{array}{l}2467.4 \\
2548.4 \\
2577.4 \\
2615.3 \\
2623.4\end{array}$ & $\begin{array}{l}752.1 \\
776.8 \\
785.6 \\
797.1 \\
799.6\end{array}$ & $\begin{array}{c}- \\
30 \pm 5 \\
3 \pm 1 \\
2 \pm 1 \\
3 \pm 1\end{array}$ & $\begin{array}{l}2 \pm 1 \\
2 \pm 1 \\
6 \pm 2 \\
4 \pm 2 \\
2 \pm 1\end{array}$ & $\begin{array}{r}- \\
12 \pm 3 \\
50 \pm 5 \\
40 \pm 5 \\
30 \pm 5\end{array}$ & $\begin{array}{l}- \\
- \\
-\end{array}$ & $\begin{array}{l}- \\
\overline{-} \\
\overline{-}\end{array}$ & $\begin{array}{r}20 \pm 2 \\
8 \pm 2 \\
2 \pm 1 \\
5 \pm 2 \\
5 \pm 2\end{array}$ & $\begin{array}{c}10 \pm 2 \\
- \\
5 \pm 3 \\
7 \pm 3 \\
2 \pm 2\end{array}$ & $\begin{array}{l}70 \pm 5 \\
50 \pm 5 \\
35 \pm 5 \\
45 \pm 5 \\
60 \pm 5\end{array}$ & $\begin{array}{l}- \\
- \\
-\end{array}$ & $\begin{array}{l}- \\
- \\
- \\
-\end{array}$ & $\begin{array}{c}1 \pm 1^{b} \\
- \\
1 \pm 1^{b} \\
- \\
=\end{array}$ \\
\hline $\begin{array}{l}2656.6 \\
2695.7 \\
2727.4\end{array}$ & $\begin{array}{r}809.7 \\
821.7 \\
\text { recture } \\
831.3 \\
\text { vein }\end{array}$ & $\begin{array}{c}4 \pm 2 \\
-1 \\
- \\
1 \pm 1 \\
1 \pm 1\end{array}$ & $\begin{array}{l}5 \pm 2 \\
3 \pm 1 \\
-1 \\
3 \pm 1 \\
2 \pm 1\end{array}$ & $\begin{array}{c}30 \pm 5 \\
30 \pm 5 \\
10 \pm 5 \\
= \\
=\end{array}$ & $\begin{array}{l}- \\
\overline{-} \\
-\end{array}$ & $\begin{array}{l}- \\
\overline{-} \\
\tilde{r}\end{array}$ & $\begin{array}{r}5 \pm 2 \\
7 \pm 3 \\
80 \pm 5 \\
17 \pm 3 \\
23 \pm 5\end{array}$ & $\begin{array}{r}8 \pm 2 \\
10 \pm 5 \\
10 \pm 5 \\
15 \pm 5 \\
11 \pm 2\end{array}$ & $\begin{array}{c}50 \pm 5 \\
40 \pm 5 \\
- \\
65 \pm 5 \\
63 \pm 5\end{array}$ & $\begin{array}{l}- \\
- \\
-\end{array}$ & $\begin{array}{l}- \\
- \\
- \\
-\end{array}$ & $\begin{array}{c}- \\
10 \pm 5^{\circ} \\
10 \pm 5^{\circ} \\
- \\
-\end{array}$ \\
\hline
\end{tabular}


Core

\begin{tabular}{|c|c|c|c|c|c|c|c|c|c|c|c|c|}
\hline Sample & $\begin{array}{l}\text { Depth } \\
(\text { a) }\end{array}$ & smectite & Mica & $\begin{array}{l}\text { Clino- } \\
\text { ptilolite }\end{array}$ & $\begin{array}{l}\text { Anal- } \\
\text { cime }\end{array}$ & $\begin{array}{l}\text { Tridy- } \\
\text { nite }\end{array}$ & Quartz & $\begin{array}{l}\text { Cristo- } \\
\text { balite }\end{array}$ & $\begin{array}{l}\text { Alkali } \\
\text { Foldspar }\end{array}$ & calcite & Glass & other \\
\hline \multicolumn{13}{|c|}{ USW GU-3 } \\
\hline \multirow{3}{*}{$\begin{array}{l}2914.5 \\
2971.0\end{array}$} & 888.3 & - & $3 \pm 1$ & - & - & - & $21 \pm 3$ & $7 \pm 3$ & $65 \pm 5$ & - & - & - \\
\hline & 905.6 & $1 \pm 1$ & $3 \pm 2$ & - & - & - & $15 \pm 3$ & $12 \pm 3$ & $70 \pm 5$ & - & - & - \\
\hline & Fracture & - & $1 \pm 1$ & $\mathbf{T r}$ & - & - & $56 \pm 5$ & $5 \pm 3$ & $38 \pm 5$ & - & - & - \\
\hline \multirow[t]{2}{*}{3004.5} & 915.8 & - & $3 \pm 2$ & - & - & - & $30 \pm 5$ & $4 \pm 2$ & $65 \pm 5$ & - & - & - \\
\hline & Fracture & - & $4 \pm 2$ & - & - & - & $17 \pm 3$ & $2 \pm 1$ & $75 \pm 5$ & - & - & - \\
\hline 3045.3 & 928.2 & $1 \pm 1$ & $3 \pm 1$ & - & - & - & $21 \pm 3$ & $7 \pm 3$ & $65 \pm 5$ & - & - & - \\
\hline 3113.1 & 948.9 & $1 \pm 1$ & $4 \pm 2$ & - & - & - & $12 \pm 3$ & $17 \pm 3$ & $65 \pm 5$ & - & - & - \\
\hline 3164.1 & 964.4 & $2 \pm 1$ & $4 \pm 2$ & $10 \pm 5$ & - & - & $9 \pm 3$ & $15 \pm 5$ & $60 \pm 5$ & - & - & - \\
\hline \multirow[t]{2}{*}{3207.4} & 977.6 & $2 \pm 1$ & $3 \pm 1$ & $10 \pm 5$ & - & - & $9 \pm 3$ & $17 \pm 3$ & $60 \pm 5$ & - & - & - \\
\hline & Fracture & $4 \pm 1$ & $6 \pm 1$ & $15 \pm 5$ & - & - & $16 \pm 2$ & $15 \pm 5$ & $45 \pm 5$ & - & - & - \\
\hline 3226.0 & 983.3 & $5 \pm 1$ & $3 \pm 1$ & $6 \pm 4$ & - & - & $12 \pm 3$ & $15 \pm 5$ & $60 \pm 5$ & - & - & - \\
\hline \multirow{3}{*}{3239.0} & 987.3 & - & $2 \pm 1$ & - & - & - & $16 \pm 2$ & $15 \pm 5$ & $65 \pm 5$ & - & - & - \\
\hline & Fracture \#1 & - & $2 \pm 1$ & - & - & - & $32 \pm 3$ & $11 \pm 4$ & $55 \pm 5$ & - & - & $6 \pm 4^{2}$ \\
\hline & Fracture $\$ 2$ & - & - & - & - & - & $41 \pm 5$ & - & $38 \pm 5$ & - & - & $20 \pm 10^{\circ}$ \\
\hline 3311.0 & 1009.2 & $3 \pm 1$ & $3 \pm 1$ & $15 \pm 5$ & - & - & $14 \pm 2$ & $10 \pm 5$ & $55 \pm 5$ & - & - & - \\
\hline 3475.3 & 1059.3 & $3 \pm 1$ & $4 \pm 1$ & $20 \pm 5$ & - & - & $14 \pm 2$ & $10 \pm 5$ & $50 \pm 5$ & - & - & - \\
\hline 3589.4 & 1094.1 & $7 \pm 3$ & $3 \pm 2$ & $35 \pm 5$ & - & - & $12 \pm 3$ & $7 \pm 3$ & $35 \pm 5$ & - & - & - \\
\hline 3672.0 & 1119.2 & $6 \pm 2$ & $3 \pm 2$ & $15 \pm 5$ & - & - & $16 \pm 2$ & $8 \pm 4$ & $45 \pm 5$ & - & - & $7 \pm 3^{\circ}$ \\
\hline 3759.1 & 1145.8 & $9 \pm 3$ & $4 \pm 2$ & $15 \pm 5$ & - & - & $27 \pm 3$ & - & $45 \pm 5$ & - & - & - \\
\hline 3854.8 & 1174.9 & $7 \pm 3$ & $2 \pm 1$ & $35 \pm 5$ & 一 & - & $25 \pm 5$ & - & $30 \pm 5$ & - & - & - \\
\hline 3859.3 & 1176.3 & $3 \pm 1$ & $2 \pm 1$ & $25 \pm 5$ & - & - & $27 \pm 3$ & - & $40 \pm 5$ & $5 \pm 1$ & - & - \\
\hline 3936.3 & 1199.8 & $1 \pm 1$ & $5 \pm 1$ & $10 \pm 5$ & $5 \pm 3$ & - & $30 \pm 2$ & -1 & $50 \pm 5$ & - & - & - \\
\hline 4008.3 & 1221.7 & $3 \pm 1$ & $6 \pm 2$ & - & $15 \pm 5$ & - & $32 \pm 3$ & - & $45 \pm 5$ & - & - & - \\
\hline 4117.0 & 1254.9 & $4 \pm 2$ & $\sim 1$ & - & $9 \pm 3$ & - & $35 \pm 3$ & - & $50 \pm 5$ & - & - & - \\
\hline 4240.6 & 1292.5 & $12 \pm 3$ & $3 \pm 1$ & - & - & - & $27 \pm 3$ & - & $50 \pm 5$ & $7 \pm 3$ & - & - \\
\hline $\begin{array}{l}4263.8 \\
4297.1\end{array}$ & $\begin{array}{l}1299.6 \\
1309.8\end{array}$ & $4 \pm 2$ & $2 \pm 1$ & - & $25 \pm 5$ & - & $32 \pm 3$ & - & $35 \pm 5$ & $1 \pm 1$ & - & - \\
\hline & Fracture & $4 \pm 2$ & $1 \pm 1$ & - & $7 \pm 3$ & - & $37 \pm 3$ & - & $7 \pm 3$ & $40 \pm 5$ & - & - \\
\hline 4416.0 & 1346.0 & $4 \pm 2$ & $1 \pm 1$ & - & $7 \pm 3$ & - & $37 \pm 3$ & - & $9 \pm 3$ & $40 \pm 5$ & - & - \\
\hline 4423.0 & 1348.1 & $9 \pm 3$ & $2 \pm 1$ & $12 \pm 3$ & $7 \pm 3$ & - & $32 \pm 3$ & - & $35 \pm 5$ & $2 \pm 1$ & - & - \\
\hline 4503.7 & 1372.7 & $20 \pm 5$ & $2 \pm 1$ & $4 \pm 2$ & $4 \pm 2$ & - & $37 \pm 3$ & - & $35 \pm 5$ & - & - & - \\
\hline & Fracture & $7 \pm 3$ & $1 \pm 1$ & $3 \pm 1$ & - & - & $62 \pm 3$ & - & $30 \pm 5$ & - & - & - \\
\hline 4568.4 & 1392.5 & $17 \pm 3$ & $3 \pm 1$ & $2 \pm 1$ & $12 \pm 3$ & - & $27 \pm 3$ & - & $35 \pm 5$ & $1 \pm 1$ & - & - \\
\hline 4600.3 & 1402.2 & $6 \pm 2$ & $2 \pm 1$ & $2 \pm 1$ & - & - & $51 \pm 3$ & - & $40 \pm 5$ & - & - & - \\
\hline 4708.5 & 1435.2 & $9 \pm 3$ & $2 \pm 1$ & - & $17 \pm 3$ & - & $34 \pm 2$ & - & $35 \pm 5$ & $2 \pm 1$ & - & - \\
\hline
\end{tabular}


APPENDIX A (cont)

Core

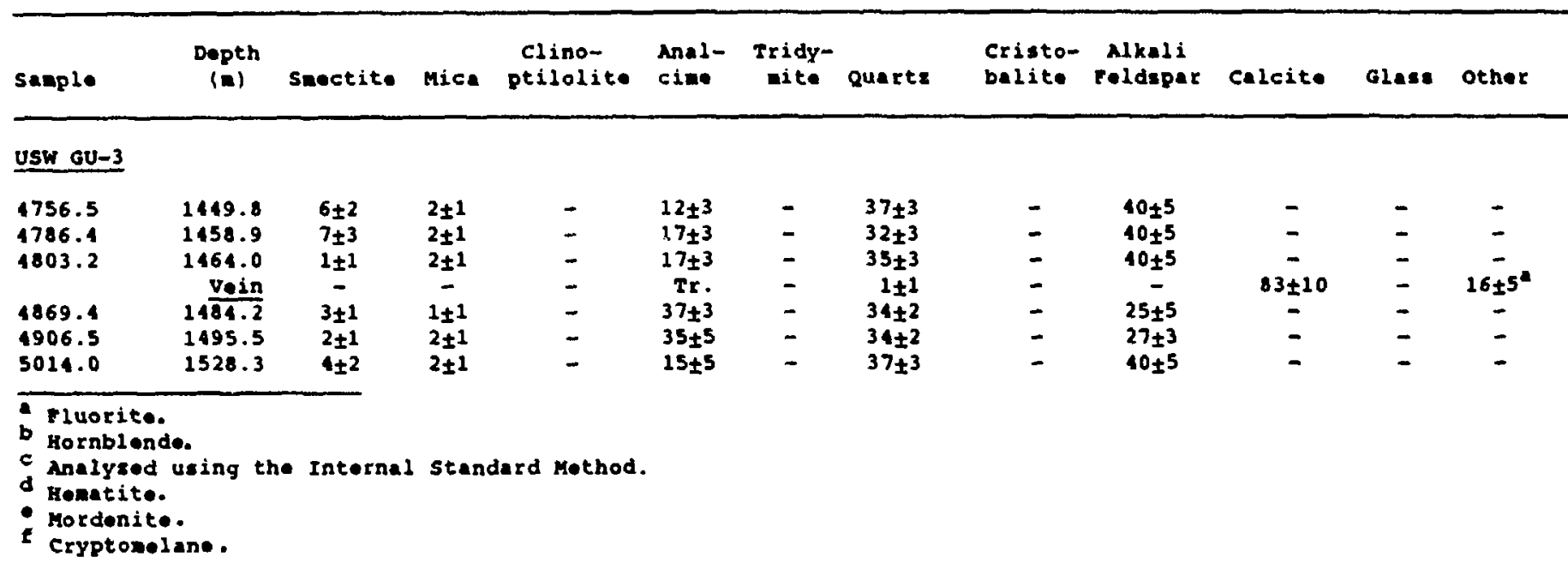


Core

\begin{tabular}{|c|c|c|c|c|c|c|c|c|c|c|c|}
\hline samplo & $\begin{array}{c}\text { Dopth } \\
\text { (I) }\end{array}$ & snectite & Mica & $\begin{array}{l}\text { Clino- } \\
\text { ptilolite }\end{array}$ & $\begin{array}{l}\text { Morden- } \\
\text { ite }\end{array}$ & $\begin{array}{l}\text { Tridy- } \\
\text { nite }\end{array}$ & Quartz & $\begin{array}{l}\text { Cristo- } \\
\text { balite }\end{array}$ & $\begin{array}{l}\text { Mkali } \\
\text { roldspar }\end{array}$ & Glass & other \\
\hline \multicolumn{12}{|l|}{ USW G-4 } \\
\hline 47 & 14.3 & $2 \pm 1$ & - & - & - & - & $2 \pm 1$ & $28 \pm 5$ & $70 \pm 10$ & - & - \\
\hline 72 & 21.9 & -1 & - & - & - & - & $2 \pm 1$ & $28 \pm 5$ & $70 \pm 10$ & - & - \\
\hline 107 & 32.6 & - & - & - & - & - & $2 \pm 1$ & $32 \pm 5$ & $67 \pm 10$ & - & - \\
\hline 123 & 37.5 & $25 \pm 10$ & - & - & - & - & - & $15 \pm 5$ & $40 \pm 10$ & $20 \pm 10$ & - \\
\hline 148 & 45.2 & $35 \pm 15$ & - & - & - & - & - & $15 \pm 10$ & - & $50 \pm 20$ & - \\
\hline 170 & 51.8 & $28 \pm 10$ & $5 \pm 2$ & - & - & - & $8 \pm 2$ & $5 \pm 2$ & $40 \pm 20$ & $20 \pm 10$ & - \\
\hline 220 & 67.1 & $10 \pm 5$ & $3 \pm 2$ & - & - & - & $4 \pm 2$ & $5 \pm 2$ & $60 \pm 20$ & $20 \pm 10$ & - \\
\hline 231 & 70.4 & $8 \pm 4$ & $10 \pm 5$ & - & - & - & $=$ & $3 \pm 2$ & $60 \pm 20$ & $20 \pm 10$ & - \\
\hline 236 & 71.9 & $=$ & - & - & - & - & $10 \pm 5$ & $10 \pm 5$ & $20 \pm 10$ & $60 \pm 20$ & - \\
\hline 268 & 81.7 & - & $\sim 1$ & - & - & $20 \pm 10$ & - & $6 \pm 2$ & $73 \pm 10$ & $=$ & - \\
\hline 280 & 85.3 & - & -1 & - & - & $10 \pm 5$ & - & $5 \pm 2$ & $80 \pm 20$ & - & - \\
\hline 332 & 101.2 & - & $2 \pm 1$ & - & - & $7 \pm 5$ & - & $15 \pm 5$ & $76 \pm 20$ & - & - \\
\hline 383 & 116.7 & $3 \pm 2$ & -1 & - & - & $15 \pm 10$ & - & $11 \pm 5$ & $70 \pm 10$ & - & - \\
\hline 410 & 125.0 & $3 \pm 2$ & -1 & - & - & $17 \pm 10$ & $\sim 1$ & $12 \pm 5$ & $66 \pm 10$ & - & - \\
\hline 416 & 126.8 & $3 \pm 2$ & $\sim 1$ & - & - & $9 \pm 5$ & -1 & $14 \pm 5$ & $73 \pm 10$ & - & - \\
\hline 447 & 136.2 & $2 \pm 1$ & $\sim 1$ & - & - & $6 \pm 4$ & $3 \pm 1$ & $20 \pm 5$ & $68 \pm 10$ & - & - \\
\hline 514 & 156.7 & $2 \pm 1$ & - & - & - & $6 \pm 4$ & $2 \pm 1$ & $22 \pm 5$ & $69 \pm 10$ & - & - \\
\hline 556 & 169.5 & $2 \pm 1$ & - & - & - & $19 \pm 10$ & $8 \pm 2$ & $9 \pm 5$ & $58 \pm 10$ & - & $4 \pm 2^{a}$ \\
\hline 625 & 190.5 & $2 \pm 1$ & - & - & - & $11 \pm 5$ & $17 \pm 4$ & $6 \pm 2$ & $63 \pm 10$ & - & - \\
\hline 676 & 206.0 & $3 \pm 1$ & - & $\sim$ & - & $4 \pm 2$ & $4 \pm 1$ & $23 \pm 5$ & $66 \pm 10$ & - & - \\
\hline 694 & 211.5 & $3 \pm 2$ & - & - & - & $17 \pm 8$ & $4 \pm 1$ & $13 \pm 2$ & $62 \pm 10$ & - & - \\
\hline 746 & 227.4 & $-\overline{1}$ & - & - & - & $=$ & $3 \pm 1$ & $28 \pm 5$ & $68 \pm 10$ & - & - \\
\hline 817 & 249.0 & $2 \pm 1$ & - & - & - & - & $25 \pm 3$ & $7 \pm 3$ & $65 \pm 10$ & - & - \\
\hline 934 & 284.7 & -1 & - & - & - & $8 \pm 4$ & $16 \pm 2$ & $11 \pm 4$ & $63 \pm 10$ & - & - \\
\hline 2026 & 312.7 & -1 & - & - & - & $s \pm 2$ & $9 \pm 2$ & $20 \pm 5$ & $66 \pm 10$ & - & - \\
\hline \multirow[t]{2}{*}{1089} & 331.9 & -1 & - & - & - & $16 \pm 8$ & $12 \pm 2$ & $10 \pm 4$ & $61 \pm 10$ & - & - \\
\hline & Fracture & - & - & - & - & $=$ & $66 \pm 5$ & $-\overrightarrow{1}$ & $32 \pm 10$ & - & - \\
\hline 1117 & 340.5 & -1 & - & - & - & - & $16 \pm 2$ & $14 \pm 4$ & $69 \pm 10$ & - & - \\
\hline \multirow[t]{2}{*}{1163} & 354.5 & - & $\mathrm{Tr}$. & - & - & - & $16 \pm 2$ & $15 \pm 4$ & $69 \pm 10$ & - & - \\
\hline & nelusion & -1 & - & - & - & - & $35 \pm 5$ & $3 \pm 2$ & $61 \pm 10$ & - & - \\
\hline 1190 & 362.7 & -1 & $\mathbf{T r}$ & - & - & - & $25 \pm 3$ & $13 \pm 4$ & $60 \pm 10$ & - & - \\
\hline 1244 & 379.2 & -1 & -1 & - & - & - & $17 \pm 3$ & $15 \pm 4$ & $67 \pm 10$ & - & - \\
\hline 1282 & $390 . B$ & -1 & -1 & $=$ & - & - & $16 \pm 3$ & $18 \pm 4$ & $65 \pm 10$ & - & - \\
\hline 12832 & $391.1-$ & & & & & & & & & & \\
\hline $1293 E$ & 394.1 & -1 & -1 & - & - & - & $6 \pm 2$ & $23 \pm 4$ & $69 \pm 10$ & - & - \\
\hline
\end{tabular}


APPENDIX A (cont)

Cor

\begin{tabular}{|c|c|c|c|c|c|c|c|c|c|c|c|}
\hline Sanple & $\begin{array}{c}\text { Depth } \\
\text { (a) }\end{array}$ & suectite & Mica & $\begin{array}{c}\text { Clino- } \\
\text { ptilolite }\end{array}$ & $\begin{array}{l}\text { Morden- } \\
\text { ite }\end{array}$ & $\begin{array}{l}\text { Tridy- } \\
\text { nite }\end{array}$ & \multirow[t]{2}{*}{ Quartz } & \multirow[t]{2}{*}{$\begin{array}{l}\text { Cristo- } \\
\text { balite }\end{array}$} & \multirow[t]{2}{*}{$\begin{array}{c}\text { Alkali } \\
\text { Feldspar }\end{array}$} & \multirow[t]{2}{*}{ Glass } & \multirow[t]{2}{*}{ other } \\
\hline \multicolumn{7}{|l|}{ USW G-4 } & & & & & \\
\hline 1299 & 395.9 & $2 \pm 1$ & $\mathbf{T r}$. & $5 \pm 2$ & - & - & $8 \pm 2$ & $23 \pm 1$ & $62 \pm 10$ & - & - \\
\hline 1301 & 396.5 & $-\overline{1}$ & -1 & $5 \pm 2$ & - & - & $9 \pm 2$ & $20 \pm 4$ & $65 \pm 10$ & - & - \\
\hline 1310 & 399.3 & -1 & -1 & $3 \pm^{2}$ & - & - & $5 \pm 2$ & $24 \pm 5$ & $65 \pm 10$ & - & - \\
\hline 1314 & 400.5 & $45 \pm 10$ & - & $28 \pm 5$ & - & - & $2 \pm 1$ & $14 \pm 4$ & $11 \pm 5$ & - & - \\
\hline 1330 & 405.4 & - & - & - & - & - & $10 \pm 5$ & - & $20 \pm 10$ & $70 \pm 20$ & - \\
\hline 1341 & 408.7 & - & - & - & - & - & $10 \pm 5$ & - & $30 \pm 10$ & $60 \pm 20$ & - \\
\hline 1372 & 418.2 & $6 \pm 2$ & $2 \pm 1$ & $1 \pm 1$ & - & - & $5 \pm 2$ & $12 \pm 5$ & $36 \pm 10$ & $10 \pm 20$ & - \\
\hline 1381 & 420.9 & $7 \pm 3$ & - & $56 \pm 10$ & - & - & $5 \pm 2$ & $4 \pm 2$ & $28 \pm 10$ & - & - \\
\hline \multirow{3}{*}{$139=$} & Inclusion & - & $4 \pm 2$ & $10 \pm 5$ & - & - & $10 \pm 5$ & $=$ & - & $75 \pm 20$ & - \\
\hline & 424.3 & $2 \pm 1$ & - & $70 \pm 15$ & - & - & $2 \pm 1$ & $4 \pm 2$ & $21 \pm 10$ & $=$ & - \\
\hline & Inclusion & $=$ & $2 \pm 1$ & - & - & - & $5 \pm 3$ & - & - & $95 \pm 20$ & - \\
\hline 1419 & 432.5 & - & $2 \pm 1$ & $30 \pm 10$ & - & - & $7 \pm 2$ & $0 \pm 3$ & $53 \pm 10$ & - & - \\
\hline 1432 & 436.5 & $3 \pm 2$ & $1 \pm 2$ & $75 \pm 15$ & - & - & $11 \pm 4$ & $7 \pm 4$ & - & - & - \\
\hline 1438 & 438.3 & $3 \pm 2$ & -1 & $75 \pm 15$ & - & - & $4 \pm 2$ & $4 \pm 2$ & $13 \pm 5$ & - & - \\
\hline 1470 & 148.1 & $3 \pm 2$ & - & $77 \pm 10$ & - & - & $6 \pm 2$ & $14 \pm 4$ & - & - & - \\
\hline 1544 & 470.6 & $3 \pm 2$ & - & $50 \pm 10$ & $20 \pm 10$ & - & $6 \pm 2$ & $12 \pm 4$ & - & - & - \\
\hline 1602 & 488.3 & - & -1 & $40 \pm 10$ & $12 \pm 5$ & - & $5 \pm 2$ & $8 \pm 4$ & $30 \pm 10$ & - & - \\
\hline 1685 & 513.6 & -1 & - & $50 \pm 10$ & $15 \pm 10$ & - & $4 \pm 2$ & $7 \pm 3$ & $19 \pm 10$ & - & - \\
\hline 1707 & 520.3 & -1 & -1 & $32 \pm 10$ & $12 \pm 5$ & - & $3 \pm 2$ & $14 \pm 4$ & $38 \pm 10$ & - & - \\
\hline 1734 & 528.5 & - & $4 \pm 2$ & $30 \pm 10$ & $10 \pm 5$ & - & $12 \pm 2$ & $15 \pm 4$ & $29 \pm 10$ & - & - \\
\hline 1763 & 537.4 & $7 \pm 3$ & - & $50 \pm 10$ & - & - & - & $32 \pm 5$ & $13 \pm 5$ & - & - \\
\hline 1779 & 542.2 & $2 \pm 1$ & - & $15 \pm 10$ & - & - & - & $10 \pm 4$ & $14 \pm 10$ & - & - \\
\hline \multirow[t]{2}{*}{1788} & 545.0 & $3 \pm 1$ & - & $28 \pm 10$ & - & - & $4 \pm 2$ & $5 \pm 2$ & $61 \pm 10$ & - & - \\
\hline & racture & - & - & - & $93 \pm 10$ & - & $7 \pm 2$ & $=$ & $=$ & - & - \\
\hline 1794 & 546.8 & - & -1 & - & - & - & $3 \pm 1$ & $29 \pm 5$ & $67 \pm 10$ & - & - \\
\hline 1841 & 561.1 & $2 \pm 1$ & -1 & - & - & - & $27 \pm 5$ & $3 \pm 2$ & $67 \pm 10$ & - & - \\
\hline 1871 & 570.3 & -1 & -1 & - & - & - & $23 \pm 5$ & $5 \pm 2$ & $69 \pm 10$ & - & - \\
\hline 1938 & 590.7 & -1 & - & - & - & - & $5 \pm 2$ & $29 \pm 4$ & $65 \pm 10$ & - & - \\
\hline 1952 & 595.0 & -1 & $\mathbf{T r}$ & - & - & - & $5 \pm 2$ & $18+4$ & $76 \pm 10$ & - & - \\
\hline $1958^{b}$ & 596.8 & $8 \pm 2$ & - & $35 \pm 3$ & $5 \pm 2$ & - & $5 \pm 1$ & $4 \pm 1$ & $46 \pm 8$ & - & - \\
\hline 1968 & 599.8 & $3 \pm 2$ & - & $15 \pm 5$ & $20 \pm 10$ & - & \pm 2 & $10 \pm 4$ & $45 \pm 10$ & - & - \\
\hline
\end{tabular}


Core

\begin{tabular}{|c|c|c|c|c|c|c|c|c|c|c|c|}
\hline Sanple & $\begin{array}{c}\text { Depth } \\
\text { (a) }\end{array}$ & seectite & Mice & $\begin{array}{l}\text { Clino- } \\
\text { ptilolite }\end{array}$ & $\begin{array}{l}\text { Morden- } \\
\text { ite }\end{array}$ & $\begin{array}{c}\text { Tridy- } \\
\text { nite }\end{array}$ & Quertz & $\begin{array}{l}\text { Cristo- } \\
\text { balite }\end{array}$ & $\begin{array}{l}\text { Mlkali } \\
\text { roldsper }\end{array}$ & Glass & other \\
\hline \multicolumn{12}{|l|}{ USW G-4 } \\
\hline 1989 & 606.2 & - & -1 & $44 \pm 10$ & - & - & $7 \pm 3$ & $15 \pm 4$ & $33 \pm 10$ & - & - \\
\hline 2039 & 621.5 & - & - & $30 \pm 10$ & $15 \pm 10$ & - & $5 \pm 2$ & $8 \pm 3$ & $42 \pm 10$ & - & - \\
\hline 2069 & 630.6 & - & - & $35 \pm 10$ & $20 \pm 10$ & - & $3 \pm 1$ & $4 \pm 2$ & $35 \pm 10$ & - & - \\
\hline 2090 & 637.0 & - & - & $10 \pm 5$ & $35 \pm 10$ & - & $5 \pm 2$ & $10 \pm 4$ & $40 \pm 10$ & - & - \\
\hline \multirow[t]{2}{*}{2100} & 640.1 & $2 \pm 1$ & - & $30 \pm 10$ & $30 \pm 10$ & - & $2 \pm 1$ & - & $35 \pm 10$ & - & - \\
\hline & acture & $=$ & - & $5 \pm 3$ & $35 \pm 10$ & - & $20 \pm 4$ & - & $40 \pm 10$ & - & - \\
\hline 2131 & 649.5 & - & -1 & $30 \pm 10$ & $20 \pm 10$ & - & $2 \pm 1$ & - & $46 \pm 10$ & $\rightarrow$ & - \\
\hline 2202 & 671.2 & - & - & $40 \pm 10$ & $10 \pm 5$ & - & $4 \pm 2$ & $7 \pm 3$ & $37 \pm 10$ & - & - \\
\hline 2226 & 678.5 & -1 & - & $35 \pm 10$ & $15 \pm 10$ & - & $6 \pm 2$ & $2 \pm 1$ & $38 \pm 10$ & $\ldots$ & - \\
\hline 2238 & 682.1 & -1 & - & $50 \pm 10$ & $25 \pm 10$ & - & $6 \pm 2$ & - & $18 \pm 10$ & - & - \\
\hline $2248^{b}$ & 685.2 & $\mathbf{T r}$ & $3 \pm 1$ & $47 \pm 3$ & - & - & $5 \pm 1$ & - & $50 \pm 8$ & - & - \\
\hline 2263 & 689.8 & - & $5 \pm 3$ & - & - & - & $19 \pm 5$ & -1 & $75 \pm 10$ & - & - \\
\hline 2285 & 696.5 & -1 & $5 \pm 3$ & - & - & - & $32 \pm 5$ & $2 \pm 1$ & $60 \pm 10$ & - & - \\
\hline \multirow{3}{*}{2343} & 714.1 & -1 & $3 \pm 2$ & - & - & - & $31 \pm 5$ & - & $65 \pm 10$ & - & - \\
\hline & ture 1 & $9 \pm 5$ & $3 \pm 2$ & - & - & - & $49 \pm 5$ & - & $39 \pm 10$ & - & - \\
\hline & ture 2 & $6 \pm 3$ & $2 \pm 1$ & - & - & - & $38 \pm 5$ & - & $54 \pm 10$ & - & - \\
\hline 2355 & 717.8 & - & $3 \pm 1$ & - & - & - & $31 \pm 5$ & - & $66 \pm 10$ & - & - \\
\hline 2381 & 725.7 & -1 & $2 \pm 1$ & - & - & - & $30 \pm 5$ & - & $67 \pm 10$ & - & - \\
\hline 2423 & 738.5 & -1 & $2 \pm 1$ & - & - & - & $26 \pm 5$ & - & $70 \pm 10$ & - & - \\
\hline 2516 & 766.9 & - & $3 \pm 1$ & - & - & - & $29 \pm 5$ & - & $68 \pm 20$ & - & - \\
\hline 2533 & 772.1 & -1 & $2 \pm 1$ & - & - & - & $31 \pm 5$ & - & $66 \pm 10$ & - & - \\
\hline 2551 & 777.5 & - & $2 \pm 1$ & - & - & - & $25 \pm 5$ & - & $73 \pm 10$ & - & - \\
\hline 2566 & 782.1 & - & $2 \pm 1$ & - & - & - & $31 \pm 5$ & - & $68 \pm 10$ & - & - \\
\hline 2598 & 791.9 & -1 & $2 \pm 1$ & - & - & - & $25 \pm 5$ & - & $71 \pm 10$ & - & - \\
\hline 2681 & 817.2 & $22 \pm 5$ & $2 \pm 1$ & - & - & - & $9 \pm 4$ & - & $67 \pm 10$ & - & - \\
\hline 2716 & 827.8 & $=$ & - & - & $80 \pm 10$ & - & $20 \pm 5$ & - & - & - & - \\
\hline 2731 & 832.4 & - & - & $13 \pm 5$ & $60 \pm 10$ & - & $27 \pm 5$ & - & - & - & - \\
\hline 2754 & 839.4 & $12 \pm 5$ & $2 \pm 1$ & $3 \pm 2$ & $23 \pm 10$ & - & $29 \pm 5$ & - & $33 \pm 10$ & - & - \\
\hline 2758 & 840.6 & - & $5 \pm 2$ & $3 \pm 2$ & $15 \pm 5$ & - & $24 \pm 5$ & - & $53 \pm 10$ & - & - \\
\hline 2762 & 841.9 & -1 & $6 \pm 2$ & $3 \pm 2$ & $15 \pm 5$ & - & $23 \pm 5$ & - & $52 \pm 10$ & - & - \\
\hline 2792 & 851.0 & - & $2 \pm 1$ & $2 \pm 1$ & $71 \pm 14$ & - & $5 \pm 1$ & - & $19 \pm 9$ & - & - \\
\hline
\end{tabular}


APPENDIX A (cont)

Core

\begin{tabular}{|c|c|c|c|c|c|c|c|c|c|c|c|}
\hline Sanplo & $\begin{array}{l}\text { Depth } \\
\text { (I) }\end{array}$ & saectite & Mica & $\begin{array}{l}\text { clino- } \\
\text { ptilolite }\end{array}$ & $\begin{array}{l}\text { Morden- } \\
\text { ite }\end{array}$ & $\begin{array}{l}\text { Tridy- } \\
\text { aite }\end{array}$ & guartz & $\begin{array}{l}\text { Cristo- } \\
\text { balite }\end{array}$ & $\begin{array}{c}\text { Alkali } \\
\text { Foldepar }\end{array}$ & Clas: & other \\
\hline \multicolumn{12}{|l|}{ USW $G-4$} \\
\hline 2823 & $\begin{array}{r}860.5 \\
\text { Precture }\end{array}$ & $\begin{array}{l}2 \pm 1 \\
7 \pm 5\end{array}$ & $\begin{array}{l}6 \pm 2 \\
2 \pm 1\end{array}$ & $\begin{array}{c}15 \pm 10 \\
5 \pm 3\end{array}$ & $\begin{array}{c}8 \pm 5 \\
60 \pm 20\end{array}$ & $\overline{-}$ & $\begin{array}{l}28 \pm 5 \\
27 \pm 5\end{array}$ & - & $\begin{array}{c}39 \pm 10 \\
-\end{array}$ & - & $=$ \\
\hline $2838^{b}$ & 865.0 & - & $1 \pm 1$ & - & - & - & $39 \pm 1$ & - & $64 \pm 8$ & - & $\operatorname{Tr} .^{c}$ \\
\hline 2840 & 865.6 & - & $6 \pm 2$ & - & - & - & $39 \pm 5$ & - & $54 \pm 10$ & - & - \\
\hline 2875 & 876.3 & - & $4 \pm 2$ & - & - & - & $35 \pm 5$ & - & $61 \pm 10$ & - & - \\
\hline $\begin{array}{l}2931^{b} \\
2947\end{array}$ & $\begin{array}{l}893.3 \\
898.2\end{array}$ & $\begin{array}{c}\mathbf{T r} \\
-\end{array}$ & $\begin{array}{l}1 \pm 1 \\
3 \pm 2\end{array}$ & - & $=$ & - & $\begin{array}{l}38 \pm 1 \\
31 \pm 5\end{array}$ & - & $\begin{array}{l}61 \pm 7 \\
66 \pm 10\end{array}$ & - & $\operatorname{Tr} .^{c}$ \\
\hline & Fracture & - & - & - & - & - & $10 \pm 5$ & - & - & - & $80 \pm 20^{d}$ \\
\hline 3000 & 9914.4 & - & $1 \pm 2$ & - & - & - & $42 \pm 5$ & - & $54 \pm 10$ & - & - \\
\hline
\end{tabular}

balcite.

Analyzed using the Internal standard Method.

d Hematite. 
Sidowall and cuttings

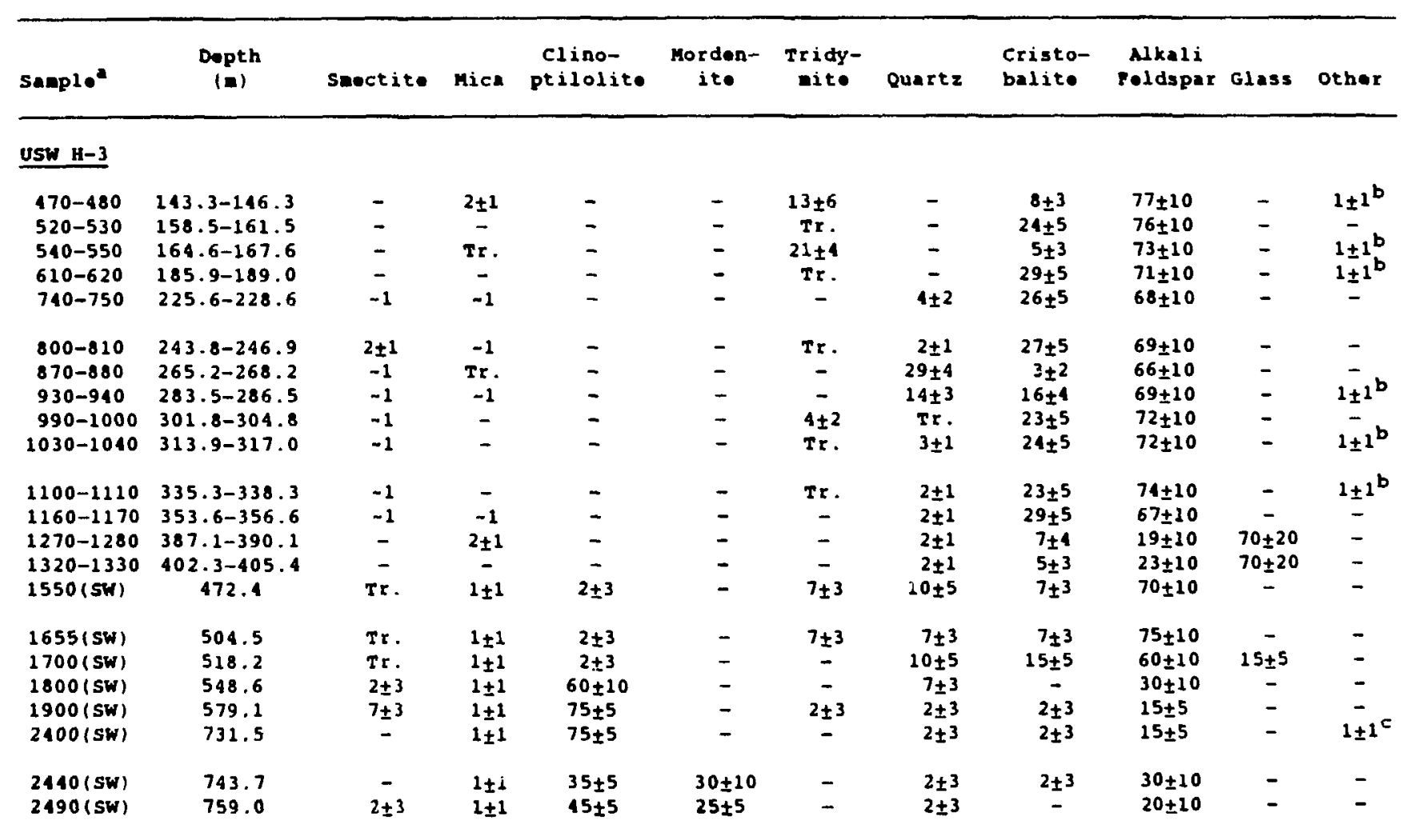

ampl• numbers represent depth of sample in foet. SW = sidewall core sample froe Levy (1984).

$b$ Henatite.

c Hotnblende. 
APPENDIX A (cont)

sidowall and cutting:

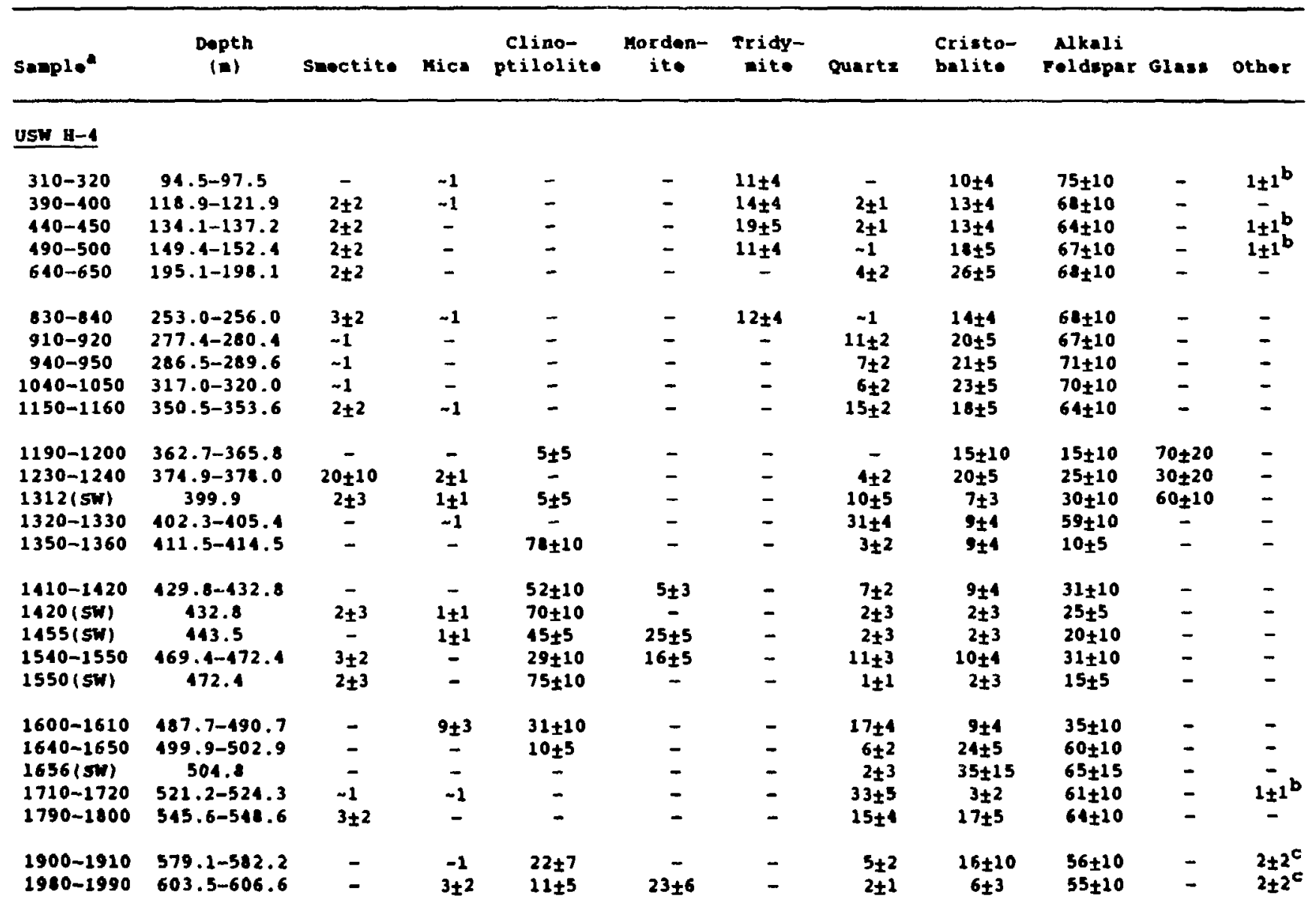

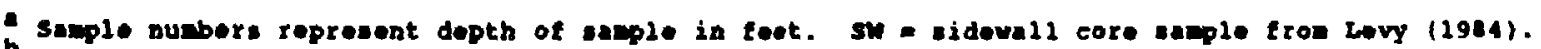

b romtite.

c Dorablendo. 
sidewall, core, and cuttings

\begin{tabular}{|c|c|c|c|c|c|c|c|c|c|c|}
\hline sanplea & $\begin{array}{l}\text { Depth } \\
\text { (I) }\end{array}$ & snoctite & Mica & $\begin{array}{l}\text { Clino- } \\
\text { ptilolite }\end{array}$ & $\begin{array}{l}\text { Tridy- } \\
\text { nite }\end{array}$ & Quartz & $\begin{array}{l}\text { Cristo- } \\
\text { balite }\end{array}$ & $\begin{array}{l}\text { Alkali } \\
\text { Fe1dspar }\end{array}$ & Glass & other \\
\hline \multicolumn{11}{|l|}{ USW H-5 } \\
\hline $50(D C)$ & 15.2 & - & - & - & $17 \pm 8$ & - & $2 \pm 3$ & $80 \pm 10$ & - & - \\
\hline $190(D C)$ & 57.9 & - & - & - & $2 \pm 3$ & - & $25 \pm 5$ & $80 \pm 10$ & - & - \\
\hline $420(D C)$ & 128.0 & $15 \pm 5$ & - & - & $=$ & $1 \pm 1$ & $12 \pm 3$ & $35 \pm 5$ & $45 \pm 5$ & $1 \pm 1^{b}$ \\
\hline $450(D C)$ & 137.2 & - & - & - & - & $1 \pm 1$ & - & $2 \pm 3$ & $96 \pm 2$ & - \\
\hline $700(D C)$ & 213.4 & Tr. & $1 \pm 1$ & - & $20 \pm 5$ & - & $15 \pm 5$ & $65 \pm 10$ & - & - \\
\hline $720-730$ & $219.5-222.5$ & $3 \pm 2$ & $\mathbf{T r}$. & - & $23 \pm 10$ & - & $9+5$ & $65 \pm 10$ & - & $1 \pm 1^{c}$ \\
\hline $800-810$ & $243.8-246.9$ & $3 \pm 2$ & - & - & TE. & $2 \pm 1$ & $24 \pm 5$ & $72 \pm 10$ & - & $1 \pm 1^{c}$ \\
\hline $830-840$ & $253.0-256.0$ & $=$ & - & - & $\mathbf{T r}$ & $=$ & $24 \pm 5$ & $76 \pm 10$ & - & $1 \pm 1^{c}$ \\
\hline $920-930$ & $280.4-283.5$ & $2 \pm 2$ & -1 & - & Tr. & $3 \pm 1$ & $24 \pm 5$ & $71 \pm 10$ & - & $=$ \\
\hline $970-980$ & $295.7-298.7$ & $2 \pm 2$ & - & - & Ir. & $2 \pm 1$ & $26 \pm 5$ & $71 \pm 10$ & - & $1 \pm 1^{c}$ \\
\hline $\begin{array}{l}1050(D C) \\
1150-1160\end{array}$ & $\begin{array}{c}320.0 \\
350.5-353.6\end{array}$ & $\begin{array}{l}\mathrm{Tr} \\
2+2\end{array}$ & $\begin{array}{l}1 \pm 1 \\
\mathrm{Tr} .\end{array}$ & - & & $\begin{array}{r}2 \pm 3 \\
26+5\end{array}$ & $\begin{array}{l}40 \pm 10 \\
10 \pm 5\end{array}$ & $\begin{array}{l}55 \pm 10 \\
62+10\end{array}$ & - & - \\
\hline $\begin{array}{l}1150-1160 \\
1230-1240\end{array}$ & $374.9-353.60$ & $\begin{array}{l}2 \pm 2 \\
2 \pm 2\end{array}$ & $\begin{array}{l}\text { Tr. } \\
\text { Tr. }\end{array}$ & - & - & $\begin{array}{r}26 \pm 5 \\
7 \pm 2\end{array}$ & $\begin{array}{l}10 \pm 5 \\
23 \pm 5\end{array}$ & $\begin{array}{l}62 \pm 10 \\
69 \pm 10\end{array}$ & - & $1 \pm 1^{c}$ \\
\hline $1290-1300$ & $393.2-396.2$ & $2 \pm 2$ & -1 & - & - & $10 \pm 2$ & $18 \pm 5$ & $\begin{array}{l}69 \pm 10 \\
71 \pm 10\end{array}$ & - & $\overline{1 \pm 1} c$ \\
\hline $1380-1390$ & $420.6-423.7$ & $2 \pm 2$ & -1 & - & - & $15 \pm 4$ & $20 \pm 5$ & $65 \pm 10$ & - & $2 \pm 1 c$ \\
\hline $1450-1460$ & $442.0-445.0$ & Tr. & -1 & - & - & $20 \pm 5$ & $8 \pm 5$ & $71 \pm 10$ & - & - \\
\hline $1490-1500$ & $454.2-457.2$ & $2 \pm 2$ & -1 & - & - & $9 \pm 2$ & $21 \pm 5$ & $68 \pm 10$ & - & $1 \pm 1^{c}$ \\
\hline $1590-1600$ & $484.6-487.7$ & $35 \pm 10$ & - & $10 \pm 5$ & - & $=$ & $17 \pm 5$ & $40 \pm 10$ & - & - \\
\hline $1610(D C)$ & 490.7 & $=$ & - & - & - & $1 \pm 1$ & $2 \pm 3$ & $7 \pm 3$ & $90 \pm 5$ & - \\
\hline $1666(S W)$ & 507.8 & $50 \pm 10$ & - & $10 \pm 5$ & - & $1 \pm 1$ & $12 \pm 3$ & $25 \pm 5$ & - & - \\
\hline $1710-1720$ & $521.2-524.3$ & $3 \pm 2$ & - & - & - & $5 \pm 3$ & $5 \pm 3$ & $20 \pm 10$ & $70 \pm 20$ & - \\
\hline 1750 (DC) & 533.4 & - & - & Tr? & - & $2 \pm 3$ & $5 \pm 5$ & $5 \pm 5$ & $85 \pm 5$ & - \\
\hline $1762(\mathrm{SW})$ & 537.1 & - & - & - & - & $1 \pm 1$ & $1 \pm 1$ & $5 \pm 5$ & $95 \pm 5$ & - \\
\hline $1800(\mathrm{SW})$ & 548.6 & - & $1 \pm 1$ & - & - & $2 \pm 3$ & $2 \pm 3$ & $10 \pm 5$ & $85 \pm 5$ & - \\
\hline $1852(\mathrm{SW})$ & 564.5 & - & $1 \pm 1$ & - & - & $2 \pm 3$ & - & $7 \pm 3$ & $95 \pm 5$ & - \\
\hline 1875 (SW) & 571.5 & - & - & Ir? & - & $2 \pm^{3}$ & Tr? & $5 \pm 5$ & $92 \pm 3$ & - \\
\hline 1917 (SH) & 584.3 & - & $1 \pm 1$ & $25 \pm 5$ & - & $30 \pm 5$ & $15 \pm 5$ & $35 \pm 10$ & $=$ & - \\
\hline $1930(D C)$ & 588.3 & $2 \pm 3$ & $1 \pm 1$ & $50 \pm 10$ & - & $15 \pm 5$ & $=$ & $30 \pm 5$ & - & - \\
\hline $1966(\mathrm{SW})$ & 599.2 & $5 \pm 5$ & $=$ & $=$ & - & $2 \pm 3$ & - & $15 \pm 5$ & $75 \pm 10$ & - \\
\hline $2200(D C)$ & 670.6 & $\mathbf{T r}$. & - & $60 \pm 10$ & - & $2 \pm 3$ & $2 \pm 3$ & $35 \pm 5$ & $=$ & - \\
\hline
\end{tabular}

- Sample numbers represent depth of sauple in foet. sw = sidevall cote sample; DC a drill (bit) b cutting ample tron Levy (19e4).

Hornblende.

c neratite. 
Appendix A (cont)

Core

\begin{tabular}{|c|c|c|c|c|c|c|c|c|c|c|}
\hline Sanple & $\underset{\text { (a) }}{\text { Depth }}$ & smoetite & Mica & $\begin{array}{l}\text { Clino- } \\
\text { ptilolite }\end{array}$ & $\begin{array}{l}\text { Anal- } \\
\text { cies }\end{array}$ & Quertz & $\begin{array}{l}\text { Cristo- } \\
\text { belite }\end{array}$ & $\begin{array}{l}\text { Alkali } \\
\text { reldaper }\end{array}$ & Gles: & other \\
\hline \multicolumn{11}{|l|}{ USW H-6 } \\
\hline 1092.4 & 333.0 & Tr. & Tr. & - & - & $20 \pm 5$ & $9 \pm 5$ & $70 \pm 20$ & - & - \\
\hline 1128.8 & 344.1 & Tr. & -1 & - & - & $21 \pm 5$ & $12 \pm 5$ & $66 \pm 10$ & - & - \\
\hline 1149.2 & 350.3 & Tr. & Tr. & - & - & $16 \pm 5$ & $15 \pm 5$ & $69 \pm 10$ & - & - \\
\hline 1166.3 & 355.5 & $3 \pm 2$ & -1 & - & - & $21 \pm 5$ & $13 \pm 5$ & $62 \pm 10$ & - & - \\
\hline 1368.4 & 117.1 & $3 \pm 2$ & $\mathbf{T r}$ & - & - & $8 \pm 3$ & $3 \pm 2$ & $25 \pm 5$ & $60 \pm 25$ & - \\
\hline 1376.2 & 419.5 & Tr & -1 & - & - & $2 \pm 1$ & $1 \pm 2$ & $32 \pm 5$ & $60 \pm 25$ & - \\
\hline 1380.8 & 420.9 & $10 \pm 5$ & - & - & - & $7 \pm 5$ & $10 \pm 5$ & $20 \pm 10$ & $60 \pm 25$ & - \\
\hline 1426.6 & 434.8 & $=$ & -1 & $6 \pm 3$ & - & $7 \pm 5$ & $10 \pm 5$ & $25 \pm 10$ & $60 \pm 25$ & - \\
\hline 1511.7 & 460.8 & $10 \pm 5$ & $2 \pm 1$ & $36 \pm 5$ & - & $7 \pm 5$ & - & $49 \pm 10$ & $10 \pm 5$ & - \\
\hline 1671.4 & 509.4 & Tr. & $\mathbf{T r}$ & - & - & $5 \pm 3$ & $23 \pm 5$ & $72 \pm 10$ & $=$ & - \\
\hline 1679.2 & 511.8 & - & Tr. & - & - & $4 \pm 2$ & $25 \pm 5$ & $72 \pm 10$ & - & - \\
\hline 1829.5 & 557.6 & - & $2 \pm 1$ & - & - & $21 \pm 5$ & $6 \pm 5$ & $71 \pm 10$ & - & - \\
\hline 2051.0 & 625.1 & $\pi r$. & $4 \pm 2$ & - & - & $20 \pm 5$ & $8 \pm 5$ & $68 \pm 10$ & - & $\mathbf{T r} \cdot \mathbf{a}$ \\
\hline 2354.6 & 717.7 & - & $2 \pm 1$ & - & - & $17 \pm 5$ & $13 \pm 5$ & $67 \pm 10$ & - & - \\
\hline 2865.0 & 073.3 & $\mathbf{T r}$. & $3 \pm 2$ & $10 \pm 5$ & - & $29 \pm 5$ & - & $50 \pm 10$ & - & - \\
\hline 3003.2 & 915.4 & - & $15 \pm 5$ & $26 \pm 5$ & - & - & $5 \pm 3$ & $51 \pm 10$ & - & $3 \pm 2$ \\
\hline 3188.4 & 971.8 & $3 \pm 2$ & $12 \pm 5$ & - & - & $5 \pm 3$ & $19 \pm 5$ & $63 \pm 10$ & - & $2 \pm 1$ \\
\hline 3605.2 & 1098.9 & Tr. & $10 \pm 5$ & $3 \pm 2$ & - & $5 \pm 3$ & $7 \pm 5$ & $58 \pm 10$ & $15 \pm 10$ & $2 \pm 1$ \\
\hline 3806.0 & 1160.1 & $3 \pm 2$ & $8 \pm 5$ & $9 \pm 5$ & $12 \pm 5$ & $22 \pm 5$ & $2 \pm 1$ & $16 \pm 10$ & - & - \\
\hline
\end{tabular}

- Hornblende. 
cuttings

\begin{tabular}{|c|c|c|c|c|c|c|c|c|c|c|c|c|}
\hline Sanple & $\begin{array}{c}\text { Depth } \\
(\mathbf{a})\end{array}$ & saectite & Mice & $\begin{array}{l}\text { Clino- } \\
\text { ptilolite }\end{array}$ & $\begin{array}{l}\text { Morden- } \\
\text { ite }\end{array}$ & $\begin{array}{l}\text { Tridy- } \\
\text { aite }\end{array}$ & Quartz & $\begin{array}{l}\text { Cristo- } \\
\text { balite }\end{array}$ & $\begin{array}{c}\text { Alkali } \\
\text { Feldspar }\end{array}$ & calcite & Glass & Bematite \\
\hline \multicolumn{13}{|l|}{ USW wT-1 } \\
\hline $440-450$ & $134.1-137.2$ & $5 \pm 3$ & - & - & - & $8 \pm 4$ & - & $4 \pm 2$ & $12 \pm 4$ & - & $70 \pm 20$ & - \\
\hline $500-510$ & $152.4-155.4$ & 2 & $4 \pm 2$ & - & - & $11 \pm 5$ & - & $12 \pm 4$ & $72 \pm 10$ & $2 \pm 1$ & $=$ & $1 \pm 1$ \\
\hline $550-560$ & $167.6-170.7$ & - & $1 \pm 1$ & - & - & $19 \pm 4$ & - & $10 \pm 3$ & $71 \pm 10$ & $=$ & - & $1 \pm 1$ \\
\hline $640-650$ & $195.1-198.1$ & $1 \pm 1$ & - & - & - & $11 \pm 4$ & $9 \pm 2$ & $16 \pm 3$ & $62 \pm 10$ & - & - & $1 \pm 1$ \\
\hline $690-700$ & $210.3-213.4$ & $1 \pm 1$ & - & - & - & - & $19 \pm 2$ & $19 \pm 3$ & $61 \pm 10$ & - & - & $1 \pm 1$ \\
\hline $780-790$ & $237.7-240.8$ & $1 \pm 1$ & $1 \pm 1$ & - & - & $3 \pm 2$ & $25 \pm 3$ & $9 \pm 2$ & $61 \pm 10$ & $1 \pm 1$ & - & - \\
\hline $840-850$ & $256.0-259.1$ & $1 \pm 1$ & $1 \pm 1$ & - & - & $6 \pm 3$ & $20 \pm 2$ & $16 \pm 3$ & $56 \pm 10$ & $=$ & - & $<1$ \\
\hline $930-940$ & $283.5-286.5$ & $3 \pm 2$ & $1 \pm 1$ & - & - & $5 \pm 2$ & $26 \pm 3$ & $7 \pm 2$ & $57 \pm 10$ & Tr. & - & $<1$ \\
\hline $1000-1010$ & $304.8-307.8$ & $1 \pm 1$ & $\overline{T r}$ & - & - & - & $22 \pm 2$ & $16 \pm 3$ & $60 \pm 10$ & $1 \pm 1$ & - & $<1$ \\
\hline $1090-1100$ & $332.2-335.3$ & $1 \pm 1$ & $\mathbf{T r}$ & - & - & - & $24 \pm 3$ & $15 \pm 3$ & $58 \pm 10$ & - & - & $<1$ \\
\hline $1160-1170$ & $353.6-356.6$ & $1 \pm 1$ & $1 \pm 1$ & $1 \pm 1$ & - & $11 \pm 4$ & $10 \pm 2$ & $18 \pm 3$ & $58 \pm 10$ & $1 \pm 1$ & - & $<1$ \\
\hline $1220-1230$ & $371.9-374.9$ & - & $1 \pm 1$ & $=$ & - & $5 \pm 3$ & $27 \pm 3$ & $8 \pm 2$ & $59 \pm 10$ & - & - & $<1$ \\
\hline $1300-1310$ & $396.2-399.3$ & $1 \pm 1$ & $1 \pm 1$ & $2 \pm 1$ & - & - & $35 \pm 4$ & $4 \pm 2$ & $59 \pm 10$ & - & - & $<1$ \\
\hline $1320-1330$ & $402.3-405.4$ & $2 \pm 1$ & $1 \pm 1$ & $14 \pm 4$ & - & - & $20 \pm 2$ & $13 \pm 4$ & $50 \pm 10$ & - & - & - \\
\hline $1340-1350$ & $408.4-411.5$ & $1 \pm 1$ & $1 \pm 1$ & $29 \pm 8$ & - & - & $10 \pm 2$ & $9 \pm 3$ & $51 \pm 10$ & - & - & - \\
\hline $1380-1390$ & $420.6-423.7$ & - & $1 \pm 1$ & $40 \pm 10$ & $12 \pm 4$ & - & $8 \pm 2$ & $7 \pm 3$ & $31 \pm 6$ & - & - & - \\
\hline $1410-1420$ & $429.8-432.8$ & - & $\mathbf{T r}$ & $40 \pm 10$ & $8 \pm 3$ & - & $3 \pm 2$ & $8 \pm 3$ & $40 \pm 8$ & - & - & - \\
\hline $1470-1480$ & $448.1-451.1$ & - & $1 \pm 1$ & $25 \pm 8$ & $18 \pm 5$ & - & $10 \pm 3$ & $7 \pm 3$ & $39 \pm 8$ & - & - & - \\
\hline $1510-1520$ & $460.2-463.3$ & - & $1 \pm 1$ & $43 \pm 10$ & $10 \pm 4$ & - & $8 \pm 3$ & $5 \pm 2$ & $33 \pm 7$ & - & - & - \\
\hline $1550-1560$ & $472.4-475.5$ & - & $1 \pm 1$ & $40 \pm 10$ & $12 \pm 4$ & - & $14 \pm 3$ & $7 \pm 3$ & $26 \pm 5$ & - & - & - \\
\hline $1570-1580$ & $478.5-481.6$ & - & $1 \pm 1$ & $5 \pm 3$ & $3 \pm 2$ & - & $26 \pm 3$ & $9 \pm 3$ & $57 \pm 10$ & - & - & $<1$ \\
\hline
\end{tabular}


APPERDIX A (cont)

cuttings and core

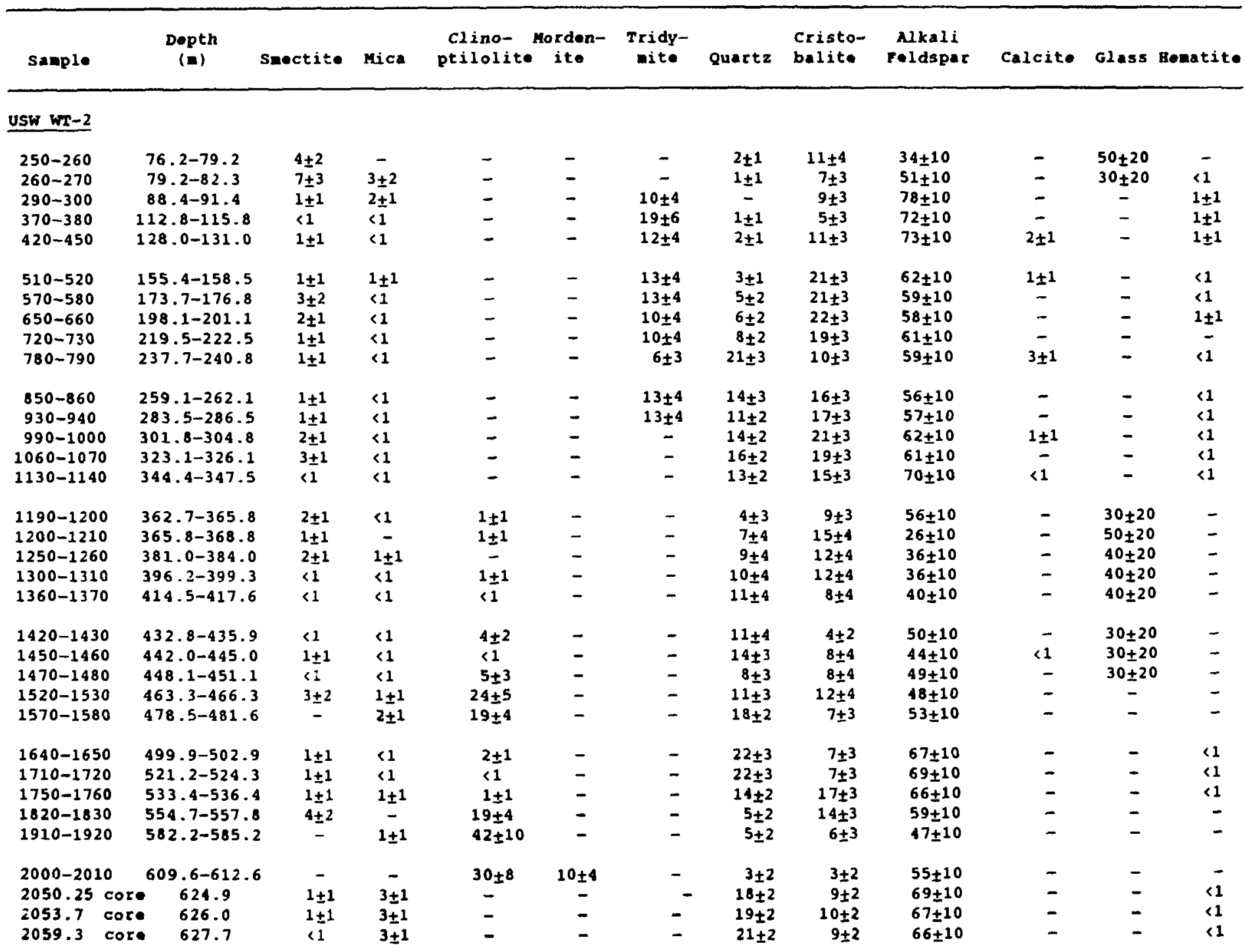




\section{APPENDIX B}

GRAPHICAL REPRESENTATION OF THE DATA IN APPENDIX A

SHOWING THE RELATIVE ABUNDANCES OF MINERAL.S AND GLASS IN DRILL. HOL.ES

AT YUCCA MOUNTAIN, NEVADA. THE SCALES ON INDIVIDUAL FIGURES CAN BE USED TO ESTIMATE RELATIVE WEIGHT PERCENTAGES. 


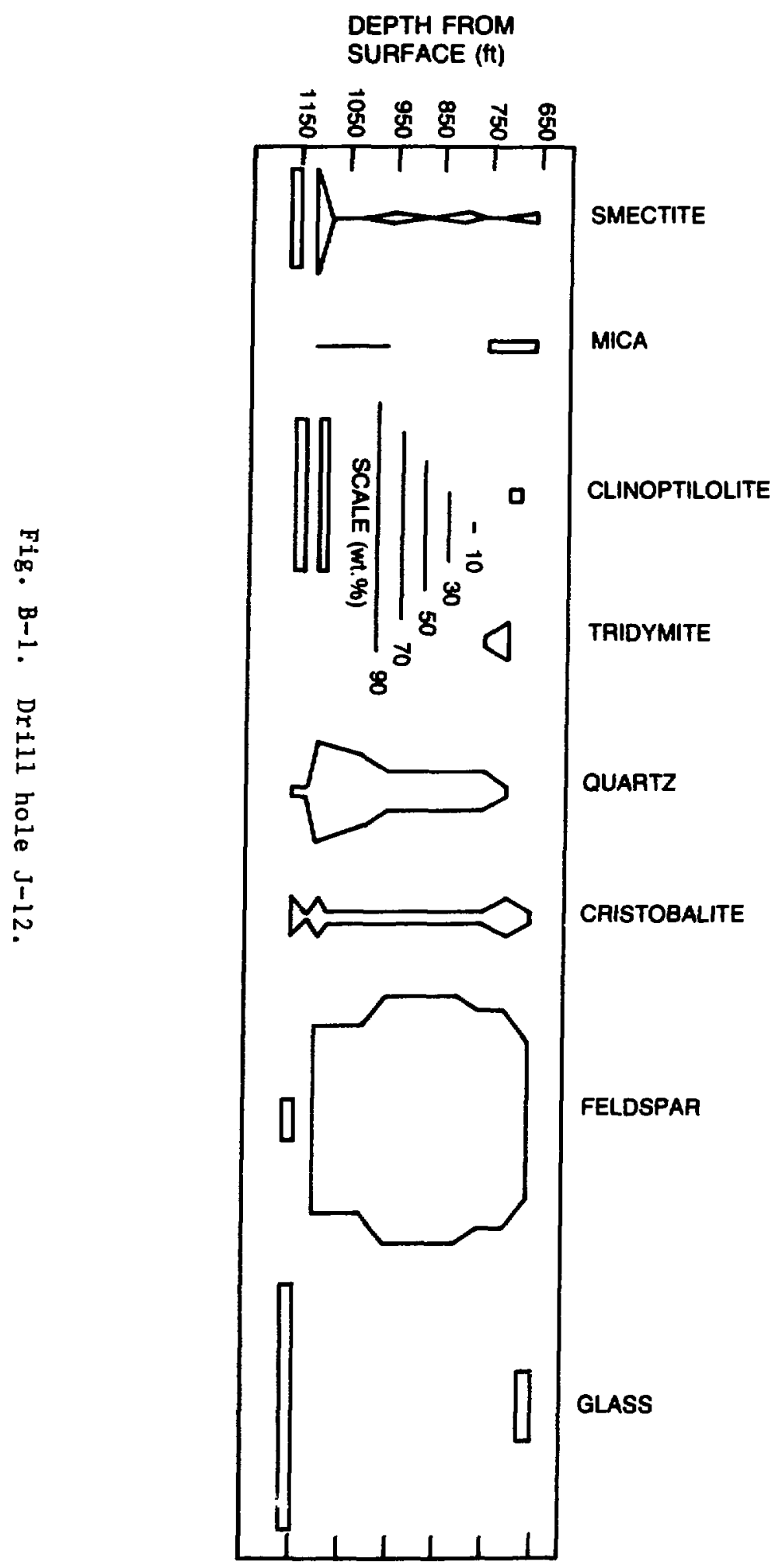




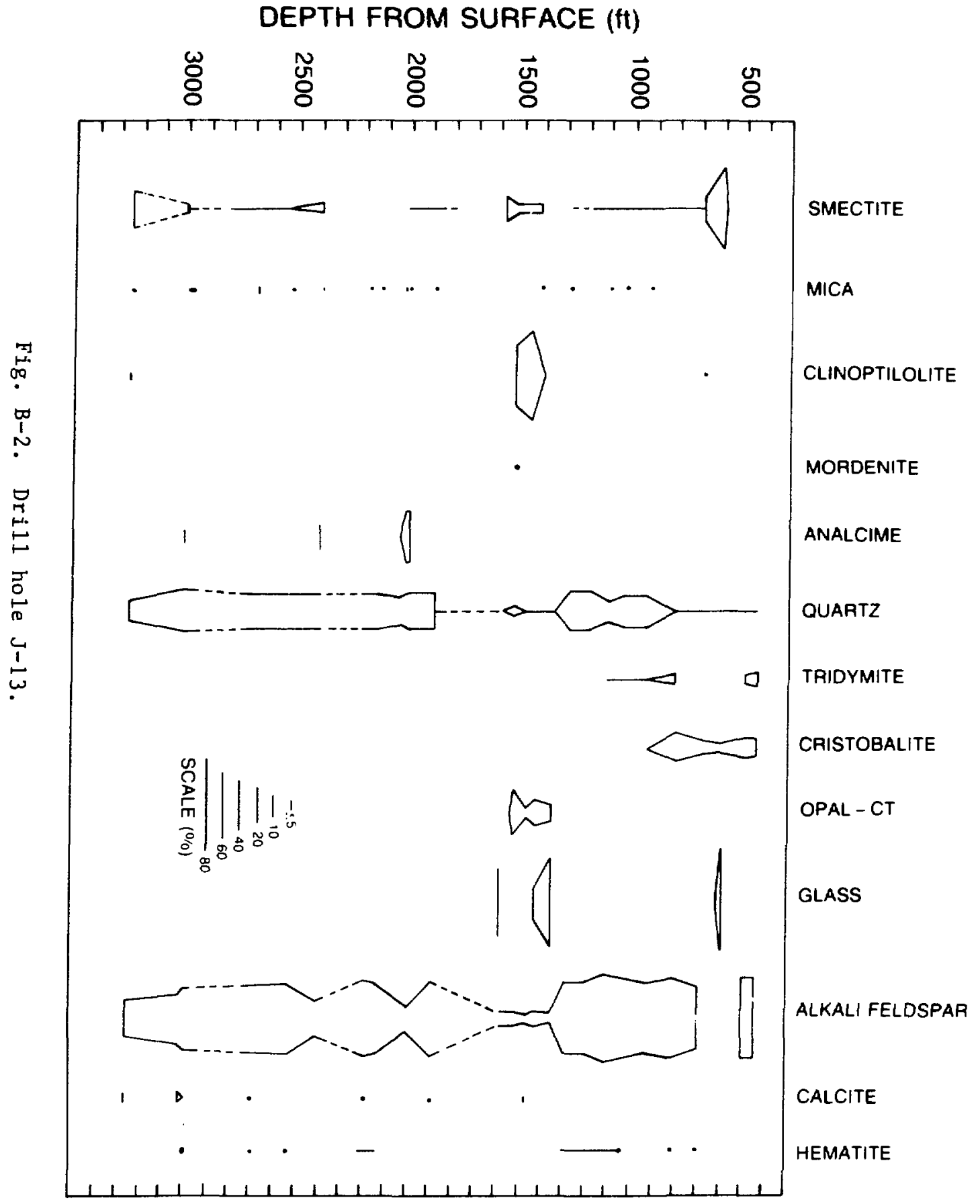




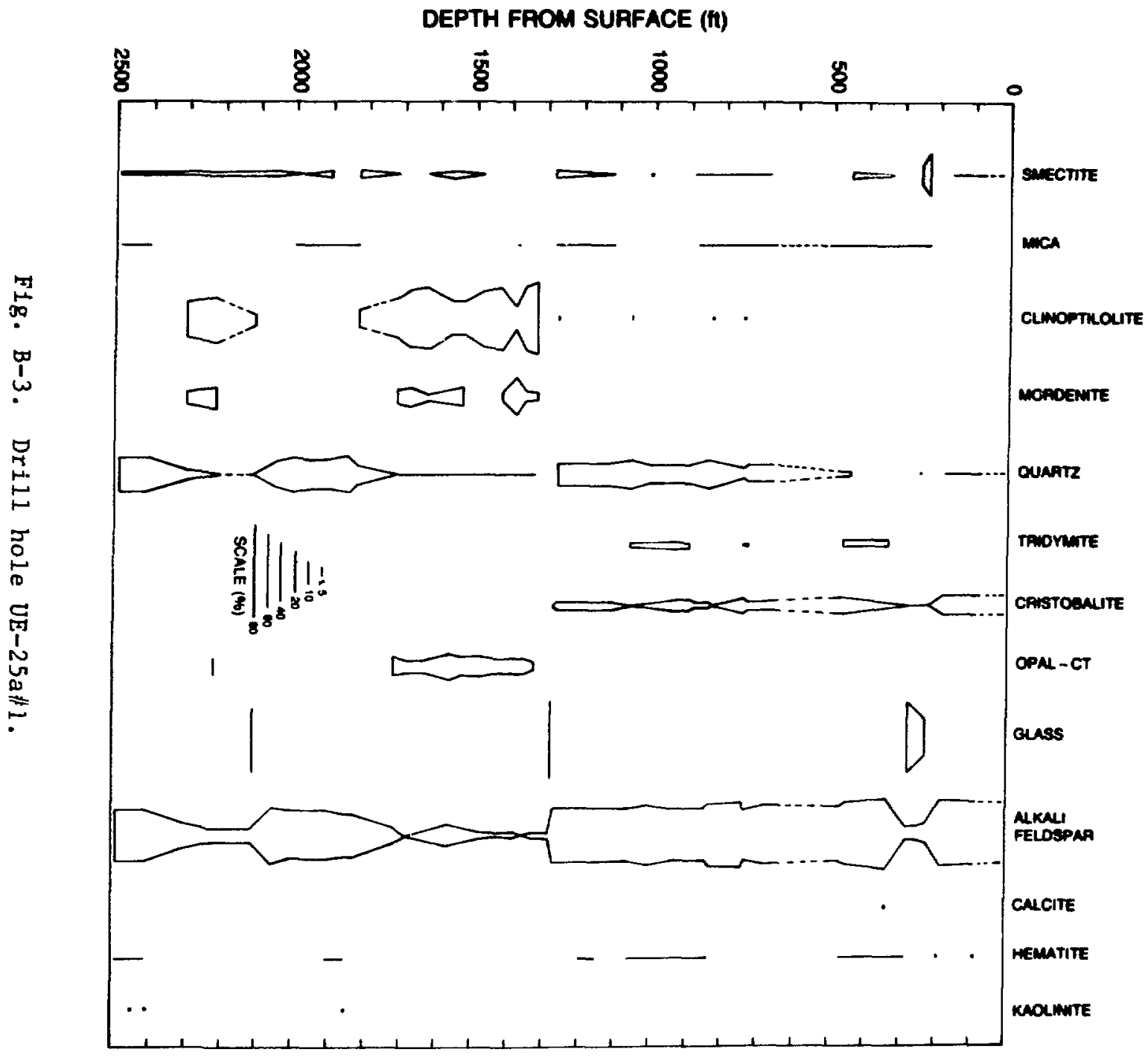


Depth From Surface (ft)

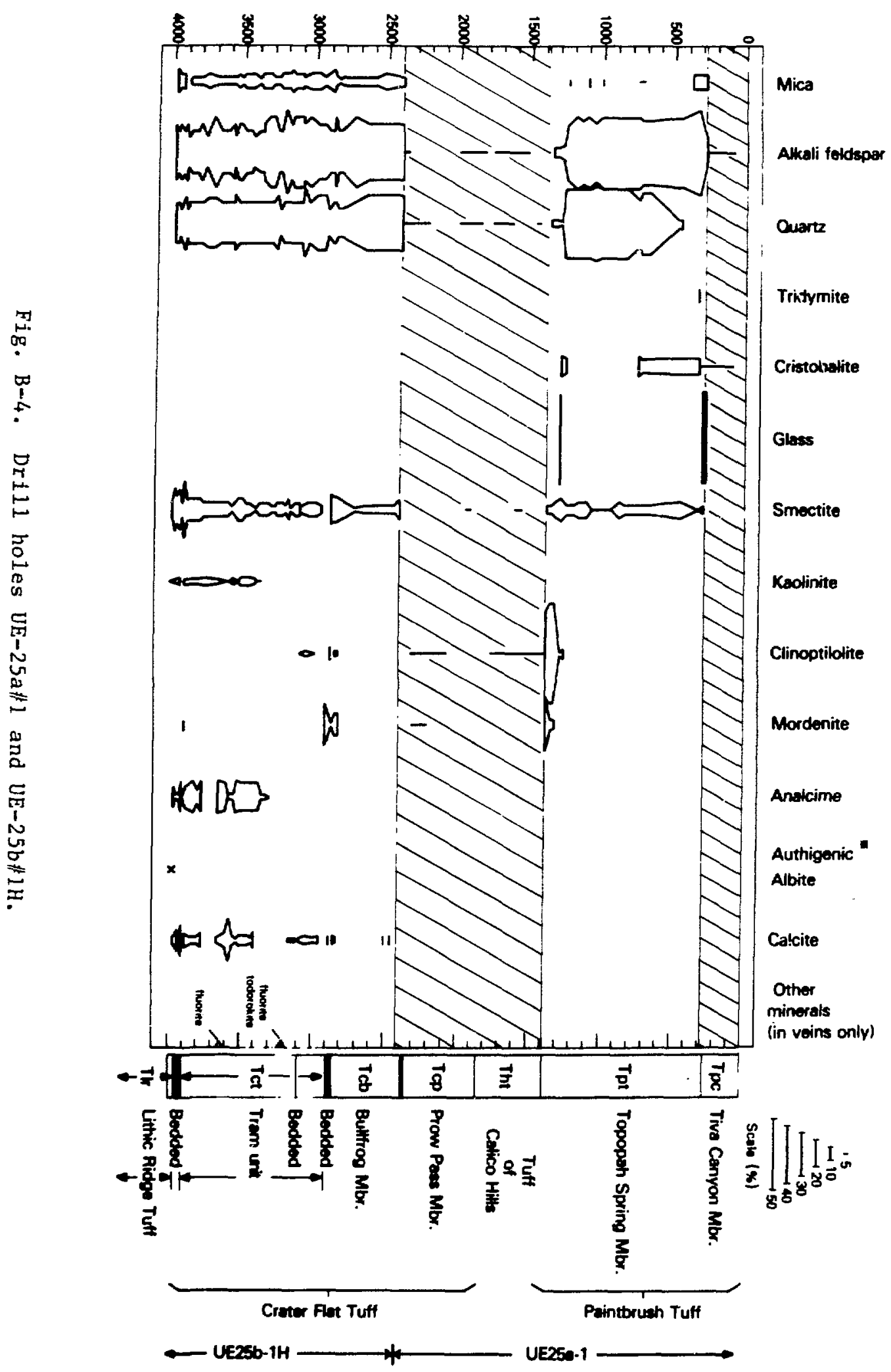




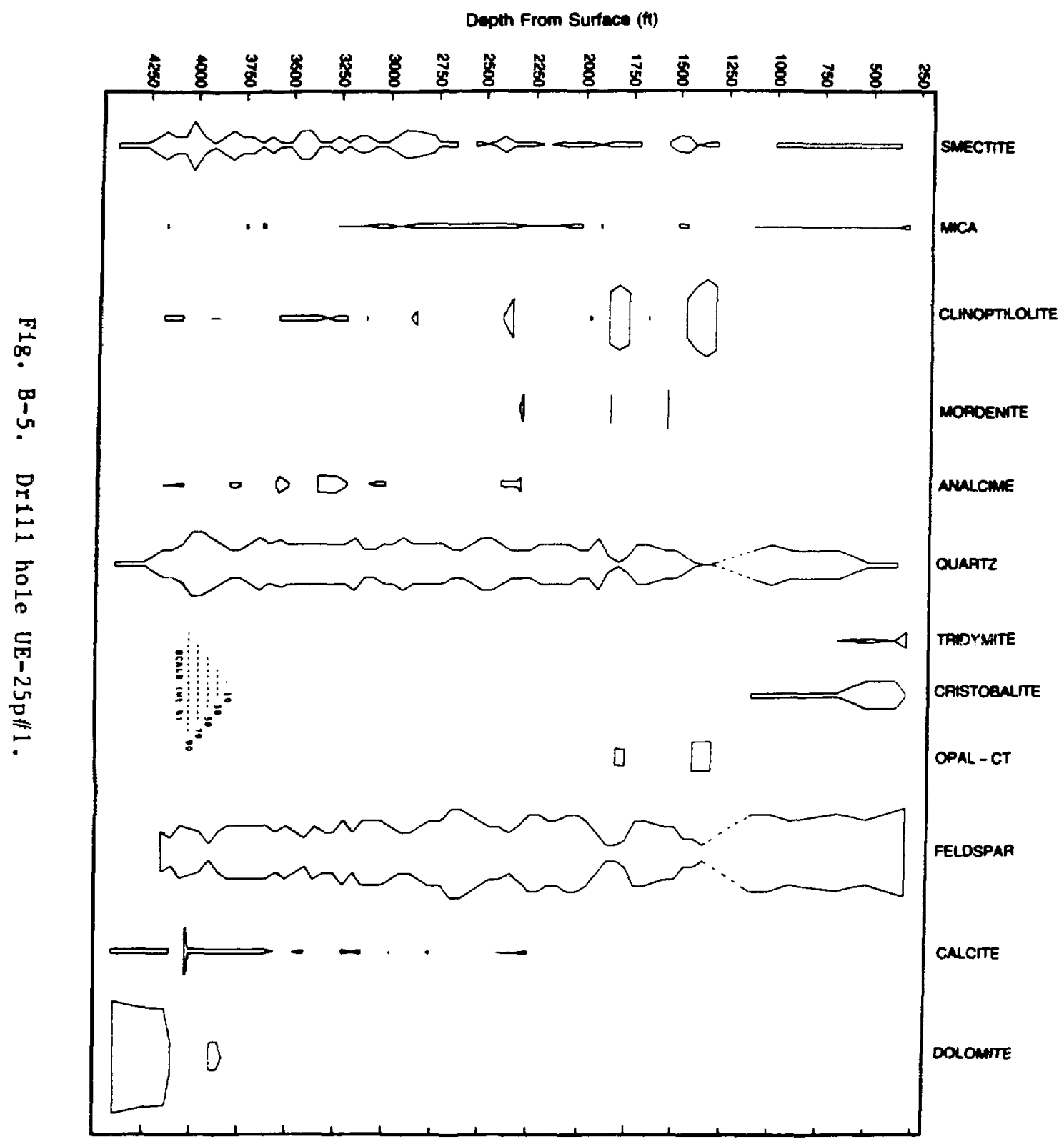




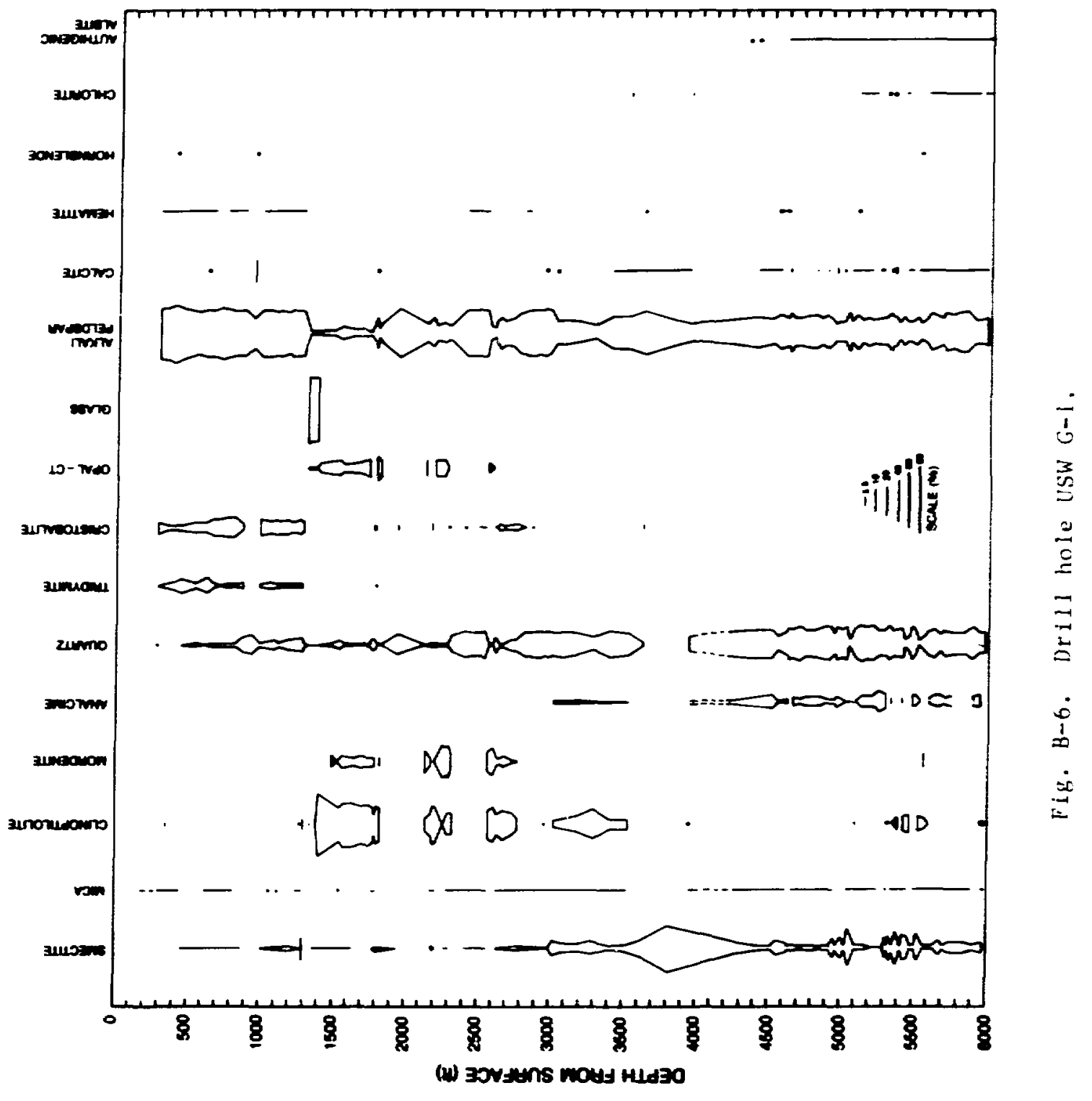




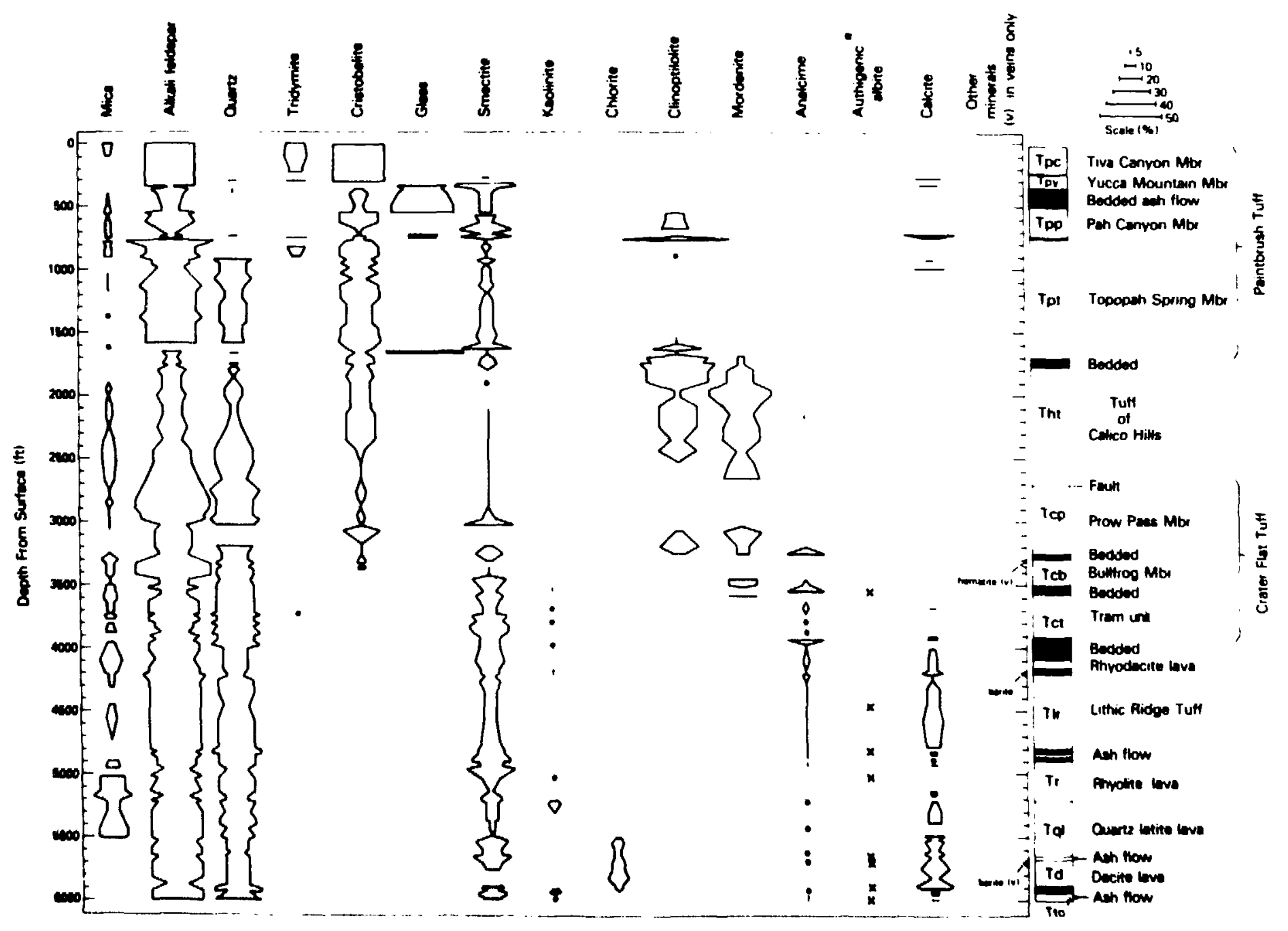




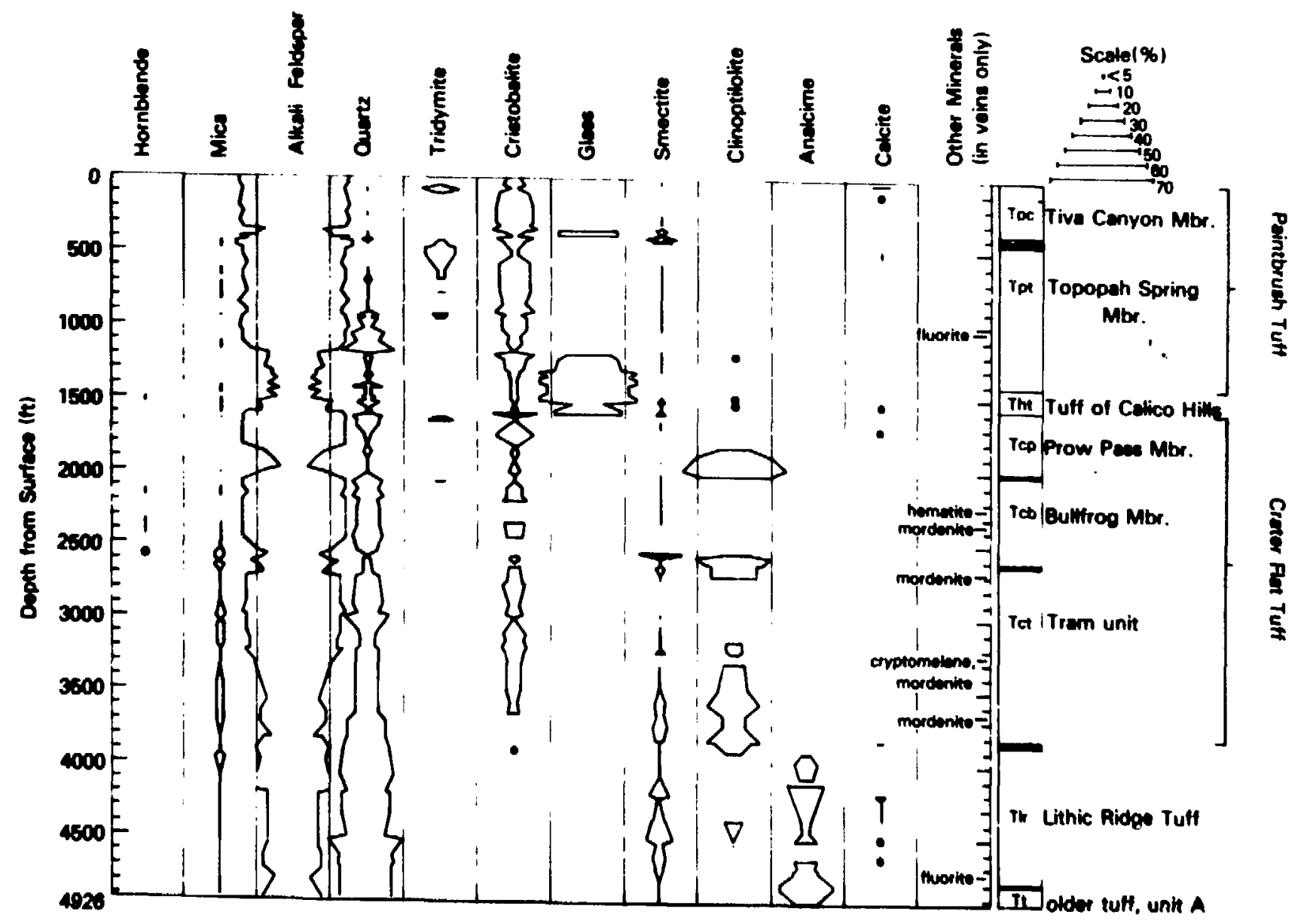

Fig. B-8. Drill holes GU-3 and G-3. 


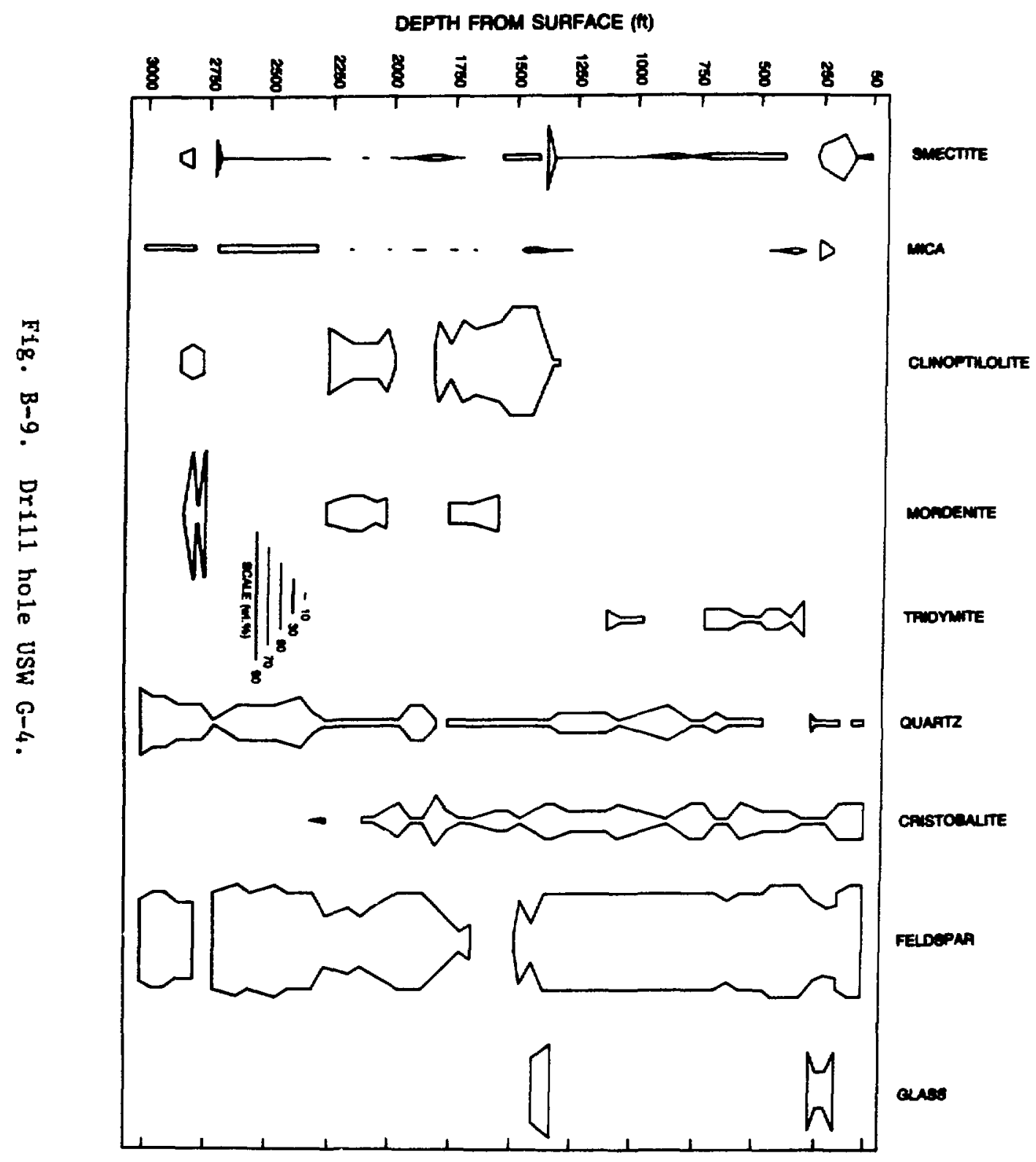




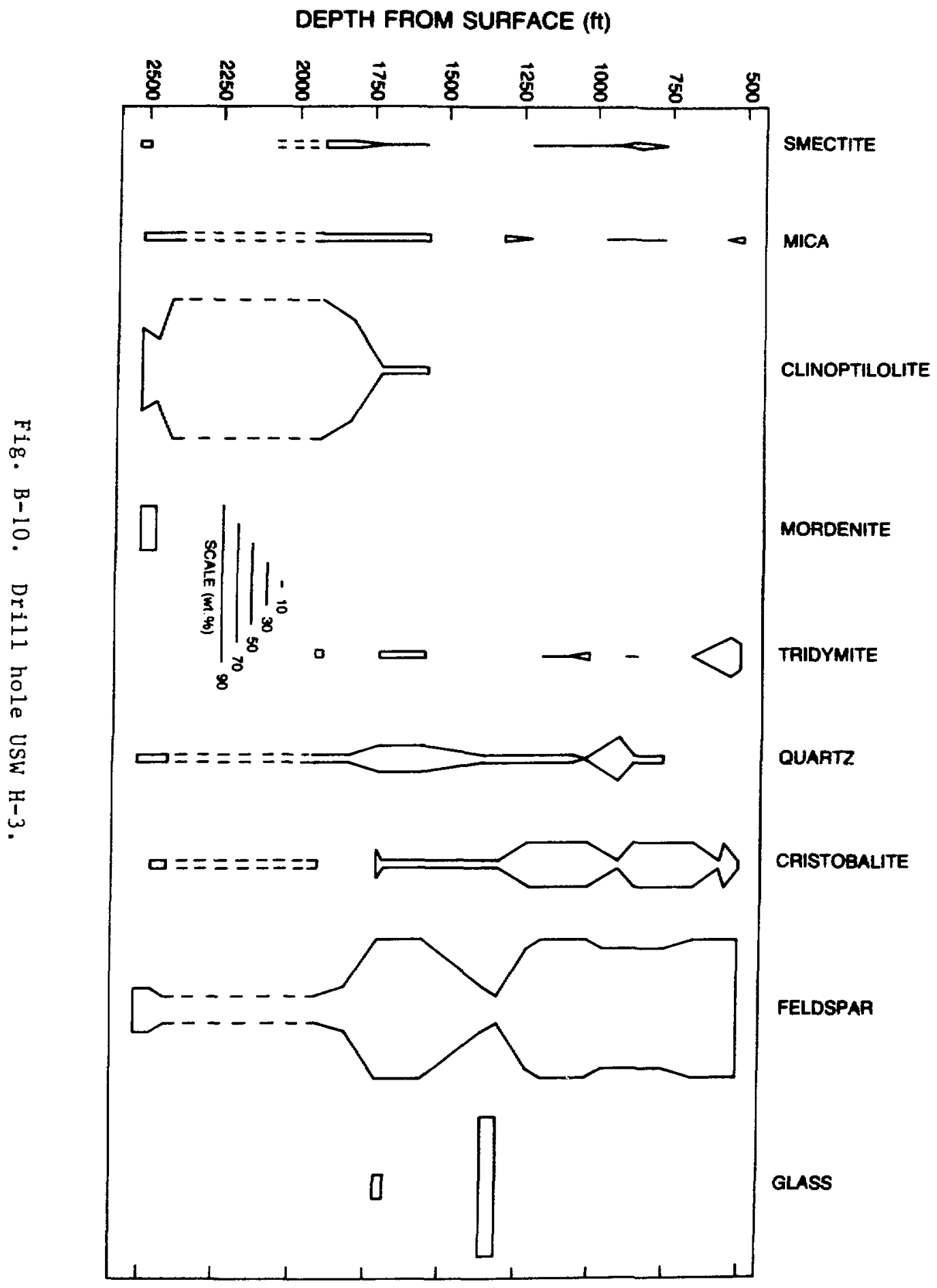




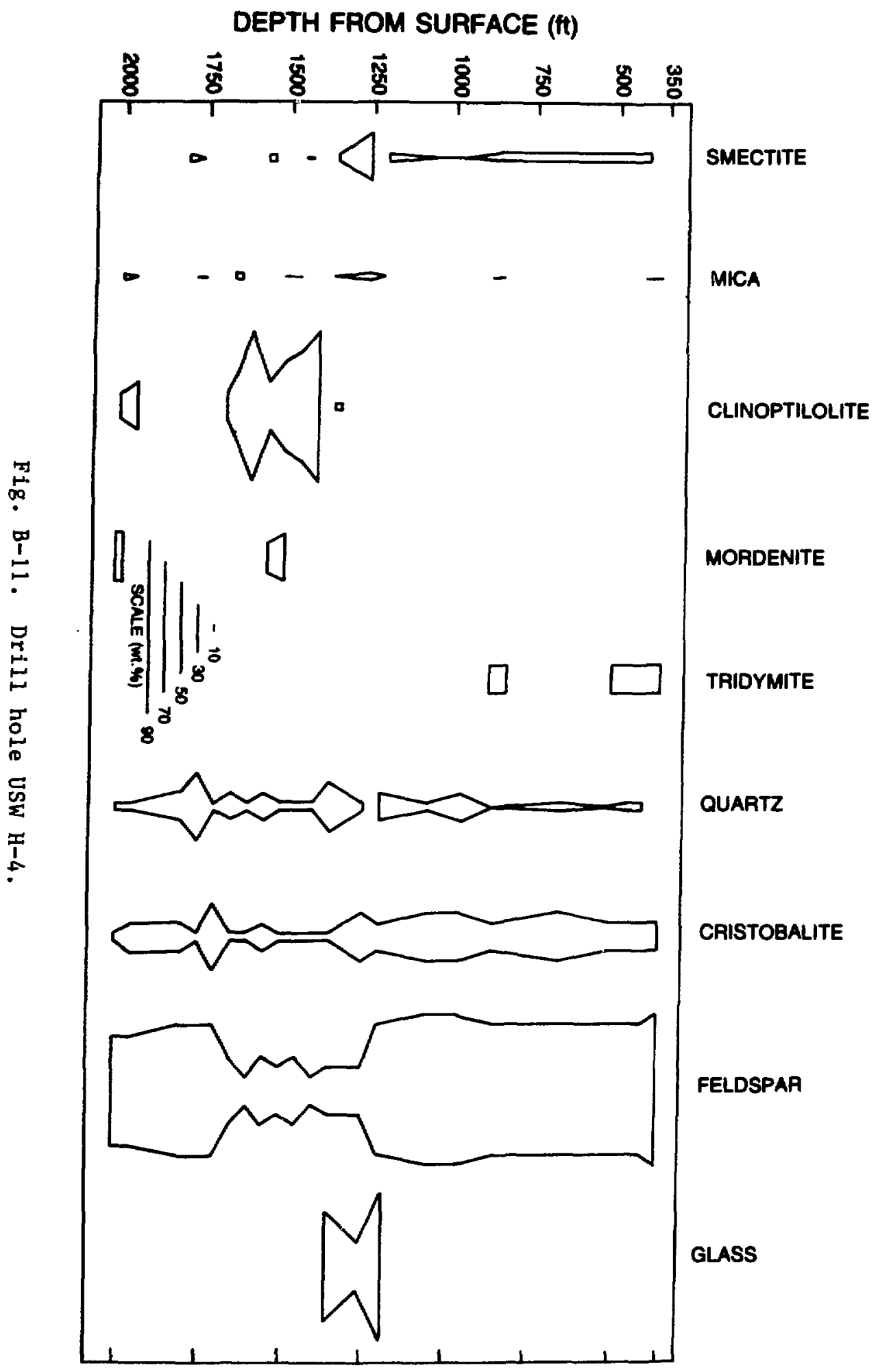




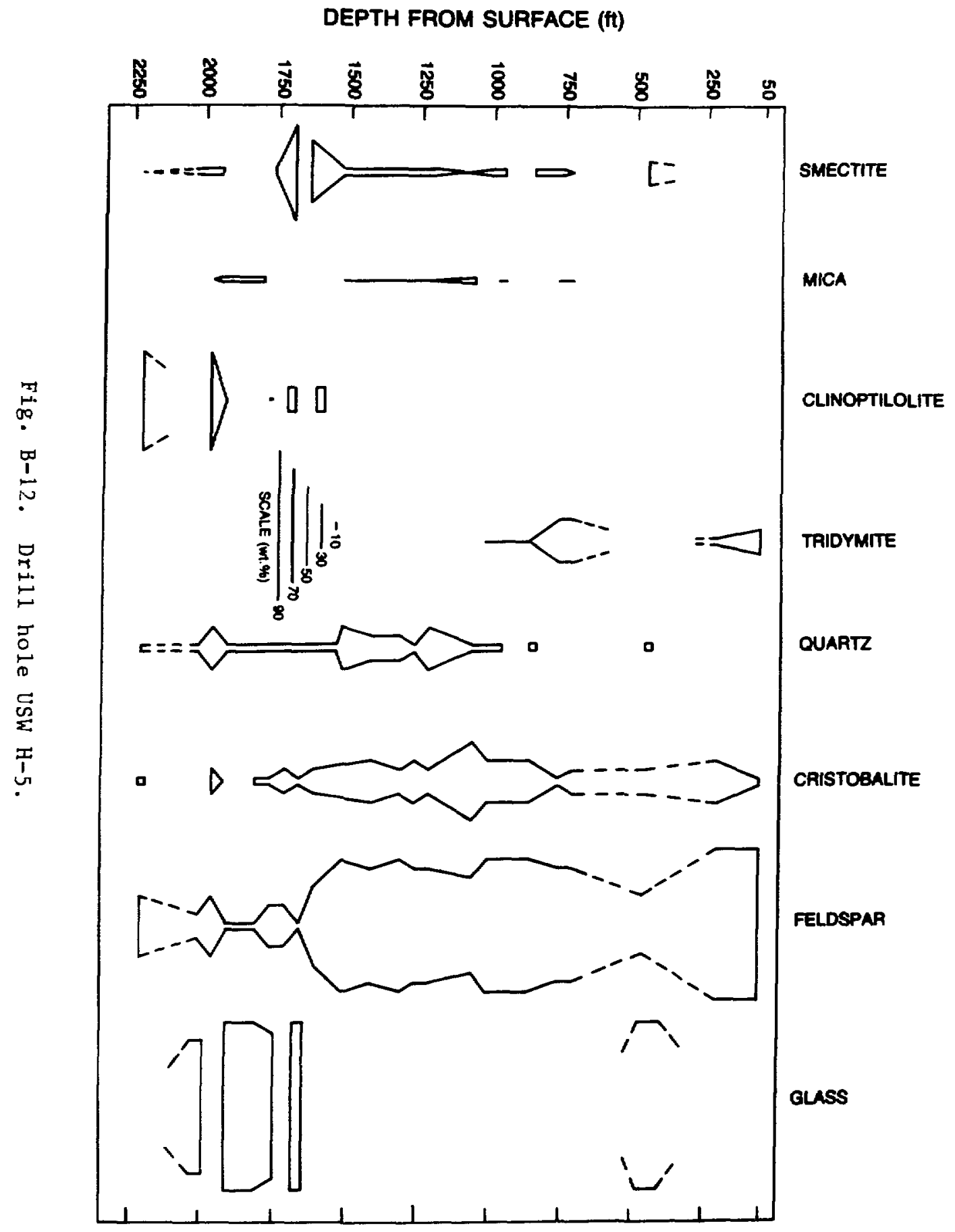




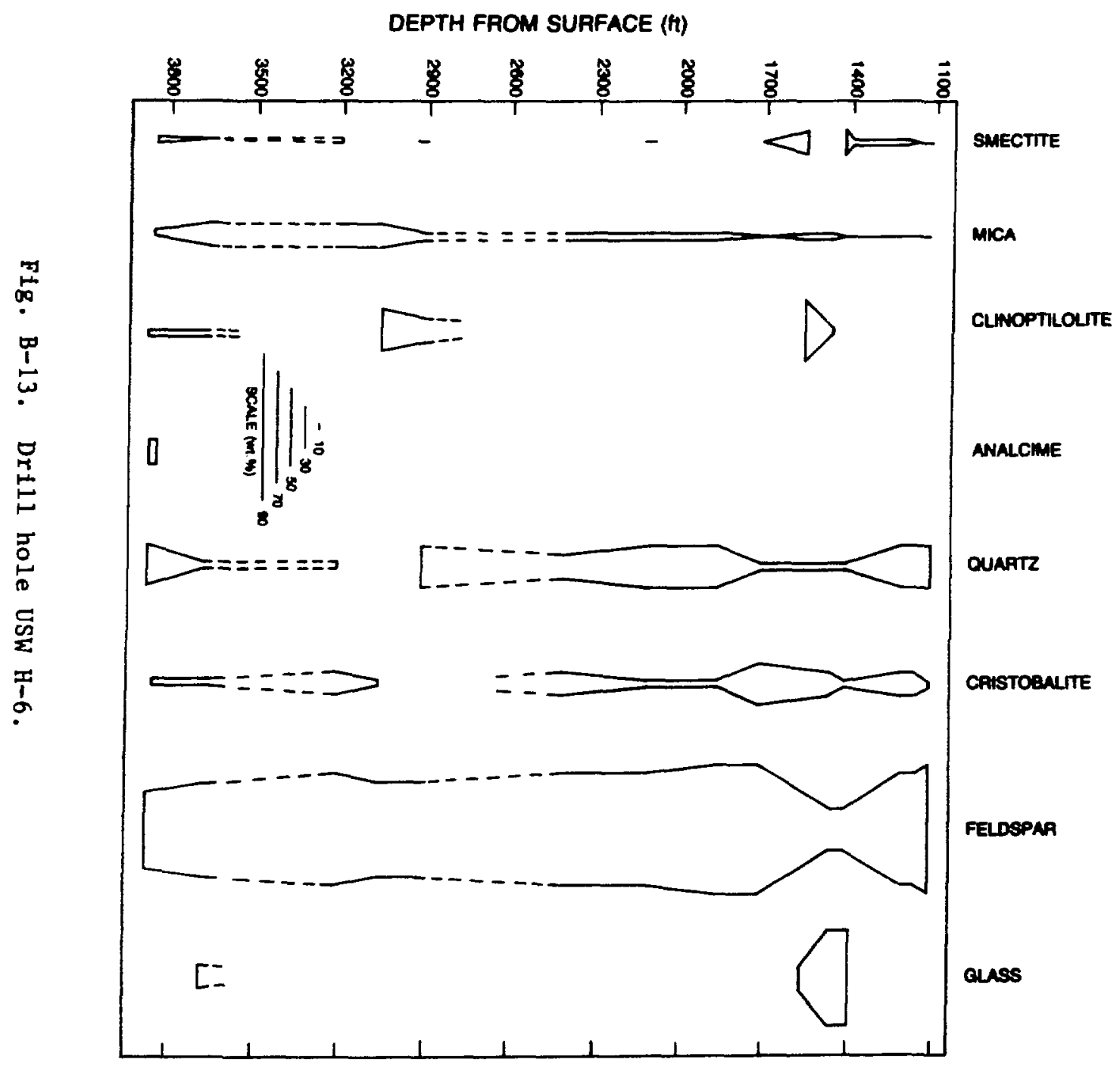


DEPTH FROM SURFACE (ft)

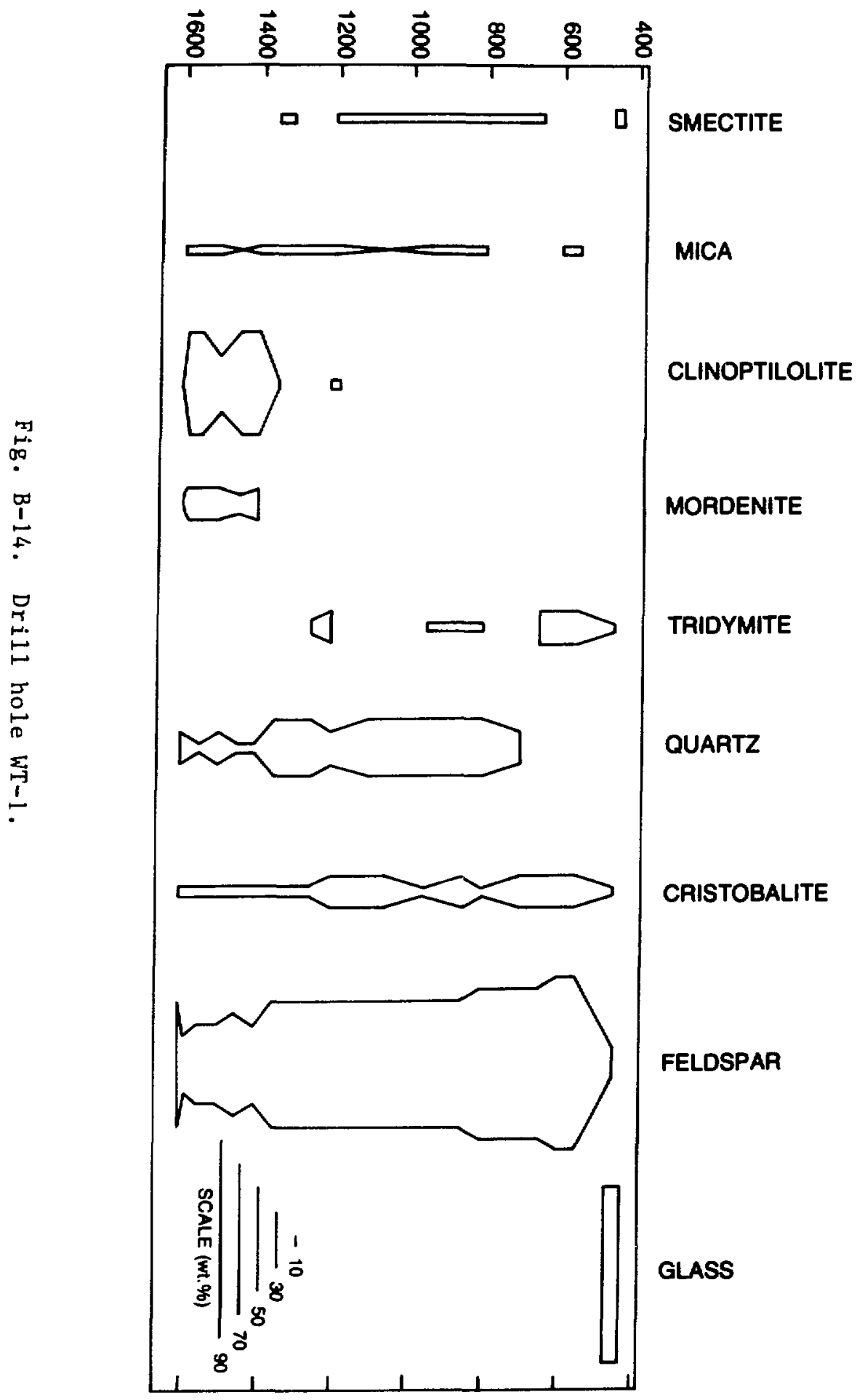




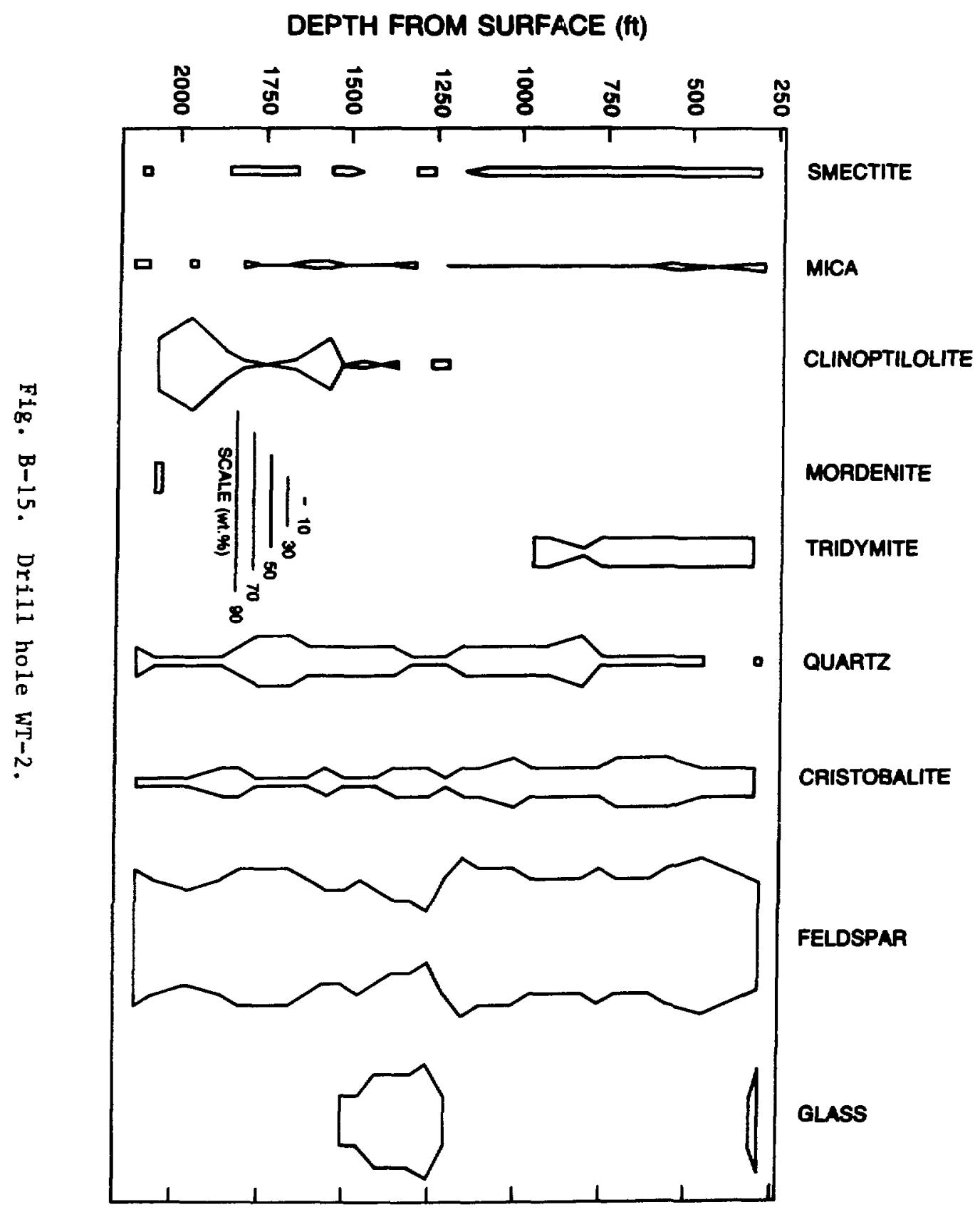




\section{REFERENCES}

Bish, D. L., "Detailed Mineralogical Characterization of the Bulffrog and Tram Members in USW-G1, with Emphasis on Clay Mineralogy," Los Alamos Nationa! Laboratory report LA-9021-MS (October 1981). NNA.870406.0238

Bish, D. L., and S. J. Chipera, "Mineralogy of Drill Holes J-13, UE-25a\#1, and USW G-1 at Yucca Mountain, Nevada," Los Alamos National Laboratory report LA-10764-MS (September 1986).

Bish, D. L., and S. J. Chipera, "Detection of Trace Amounts of Erionite in Clinoptilolite Samples Using $X$-Ray Powder Diffraction and Profile Refinement," Proceedings of the Twenty-Fourth Annual Clay Minerals Society Meeting, Socorro, New Mexico, 32 (October 1987).

Bish, D. L., and S. J. Chipera, "Problems and Solutions in Quantitative Analysis of Complex Mixtures by XRay Powder Diffraction," in Advances in X-Ray Analysis (Plenum Press, New York, 1988), Vol. 31. 295-308.

Bish, D. L., and R. E. Semarge, "Mineralogic Variations in a Silicic Tuff Sequence: Evidence for Diagenetic and Hydrothermal Reactions," Proceedings of the Nineteenth Annual Clay Minerals Society Meeting, Hilo, Hawaii, 42 (August 1982). NNA.870406.0234

Bish, D. L., and D. T. Vaniman, "Mineralogic Summary of Yucca Mountain, Nevada," Los Alamos National Laboratory report LA-10543-MS (October 1985). NNA.870407.0330

Bish, D. L., F. A. Caporuscio, J. F. Copp, B. M. Crowe, J. D. Purson, J. R. Smyth, and R. G. Warren, "Preliminary Stratigraphic and Petrologic Characterization of Core Samples from USW-G1, Yucca Mountain, Nevada," Los Alamos National Laboratory report LA-8840-MS (November 1981). NNA.870406.0158

Bish, D. L., A. E. Ogard, D. T. Vaniman, and L. Benson, "Mineralogy-Petrology and Groundwater Geochemistry of Yucca Mountain Tuffs, " in Scientific Basis for Nuclear Waste Management VII, Materials Research Society Symposia Proceedings, Boston, Massachusetts (G. L. McVay, Ed.), 283-291 (1984). NNA.870406.0085

Caporuscio, F., D. Vaniman, D. Bish, D. Broxton, B. Amey, G. Heiken, F. Byers, R. Gooley, and E. Semarge, "Petrologic Studies of Drill Cores USW-G2 and UE25b-1H, Yucca Mountain, Nevada," Los Alamos National Laboratory report LA-9255-MS (July 1982). NNA.870519.0041

Chipera, S. J., and D. L. Bish, "Mineralogy oi Drill Hole UE-25p\#1 at Yucca Mountain, Nevada," Los Alamos National Laboratory report LA-11292-MS (May 1988). NNA.880607.0036

Chung, F. H., "Quantitative Interpretation of X-Ray Diffraction Patterns of Mixtures. I. Matrix-Flushing Method for Quantitative Multicomponent Analysis," Journal of Applied Crystallography Z, 519-525 (1974a).

Chung, F. H., "Quantitative Interpretation of X-Ray Diffraction Patterns of Mixtures. II. Adiabatic Principle of X-Ray Diffraction Analysis of Mixtures," Journal of Applied Crystallography Z, 526-531 (1974b).

Ernst, W. G., and S. E. Calven, "An Experimental Study of the Recrystallization of Porcelanite and its Bearing on the Origin of Some Bedded Cherts," American Journal of Science 267A, 114-133 (1969). 
Heiken, G. H., and M. L. Bevier, "Petrology of Tuff Units from the J-13 Drill Site, Jackass Flats, Nevada," Los Alamos National Laboratory report LA-7563-MS (February 1979). NNA.870519.0045

Jones, J. B., and E. F. Segnit, "The Nature of Opal. I. Nomenclature and Constituent Phases," Journal of the Geological Society of Australia 18, 57-68 (1971).

Klug. H. P., and L. E. Alexander, X-Ray Difiraction Procedures for Polycrystalline and Amorphous Materials (John Wiley \& Sons, Inc., New York, 1974).

Levy, S. S., "Petrology of Samples from Drill Holes USW H-3, H-4, and H-5, Yucca Mountain, Nevada," Los Alamos National Laboratory report LA-9706-MS (June 1984). NNA.870519.0048

Mumpton, F. A., "Clinoptilolite Redefined," American Mineralogist 45, $351-369$ (1960).

Smith, D. K., M. C. Nichols, and M. E. Zolensky, "POWD10, A FORTRAN IV Program for Calculating XRay Powder Diffraction Patterns - Version 10," Pennsylvania State University, College of Earth and Mineral Sciences report (May 1982).

Sykes, M. L., G. H. Heiken, and J. R. Smyth, "Mineralogy and Petrology of Tuff Units from the UE25a-1 Drill Site, Yucca Mountain, Nevada," Los Alamos National Laboratory report LA-8139-MS (November 1979). NNA.870406.0186

Vaniman, D., D. Bish, D. Broxton, F. Byers, G. Heiken, B. Carlos, E. Semarge, F. Caporuscio, and R. Gooley, "Variations in Authigenic Mineralogy and Sorptive Zeolite Abundance at Yucca Mountain, Nevada, Based on Studies of Drill Cores USW GU-3 and G-3," Los Alamos National Laboratory report LA-9707-MS (June 198.4). NNA.870519.0043 
The following number is for Otfice of Civilian Radioactive Waste Management (OCRWM) Records Management purposes only and should not be used when ordering this publication.

Accession Number NNA.891019.0029

\footnotetext{
Printed in the United States of America Available from

National Technical Information Servic: US Deportment of Commerce S285 Port Royal Road Springrield, VA $2216 \mathrm{I}$

Microfiche (AO1)
}

\begin{tabular}{cc} 
Page Range & $\begin{array}{c}\text { NTIS } \\
\text { Price Code }\end{array}$ \\
\hline O01-025 & A02 \\
$026-050$ & A03 \\
$051-075$ & $A 04$ \\
$076-100$ & A0S \\
$101-125$ & A06 \\
$126-150$ & A07
\end{tabular}

\begin{tabular}{cc} 
Page Range & $\begin{array}{c}\text { NTIS } \\
\text { Price ode }\end{array}$ \\
\hline 151.175 & $A 06$ \\
176.200 & $A 09$ \\
201.225 & $A 10$ \\
226.250 & $A 11$ \\
251.275 & $A 12$ \\
276.300 & $A 13$
\end{tabular}

\begin{tabular}{cc} 
Page Range & $\begin{array}{c}\text { NTIS } \\
\text { Price Code }\end{array}$ \\
\hline 301.325 & Al4 \\
326.350 & A IS \\
351.375 & A 16 \\
376.400 & $A 17$ \\
401.425 & A 18 \\
426.450 & A 19
\end{tabular}

\begin{tabular}{cc} 
Page Range & $\begin{array}{c}\text { NTIS } \\
\text { Price Code }\end{array}$ \\
\hline 451.475 & $A 20$ \\
$476-500$ & $A 21$ \\
501.525 & $A 22$ \\
526.550 & $A 23$ \\
551.579 & $A 24$ \\
576.600 & $A 25$ \\
601.49 & $A 99$
\end{tabular}

